\title{
MARGINAL MATERIAL STABILITY
}

By

Yury Grabovsky and Lev Truskinovsky

\section{IMA Preprint Series \#2404}

(July 2012)

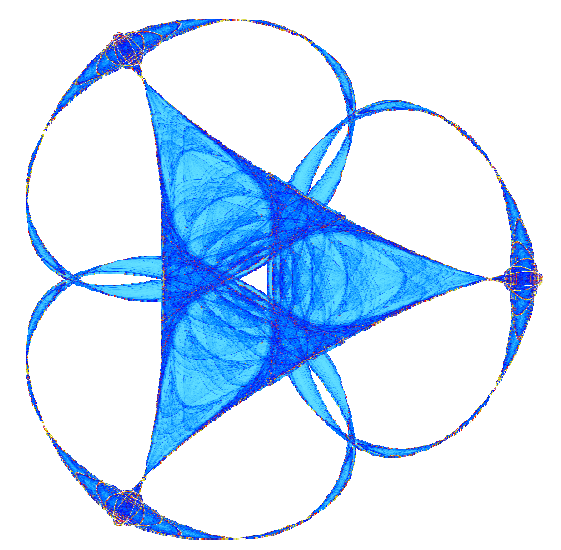

INSTITUTE FOR MATHEMATICS AND ITS APPLICATIONS UNIVERSITY OF MINNESOTA 400 Lind Hall 207 Church Street S.E.

Minneapolis, Minnesota 55455-0436

Phone: 612-624-6066 Fax: 612-626-7370

URL: http://www.ima.umn.edu 


\title{
Marginal material stability
}

\author{
Yury Grabovsky $\quad$ Lev Truskinovsky
}

July 11, 2012

\begin{abstract}
Marginal stability plays an important role in nonlinear elasticity because the associated minimally stable states usually delineate failure thresholds. In this paper we study the local (material) aspect of marginal stability. The weak notion of marginal stability at a point, associated with the loss of strong ellipticity, is classical. States that are marginally stable in the strong sense are located at the boundary of the quasi-convexity domain and their characterization is the main goal of this paper. We formulate a set of bounds for such states in terms of solvability conditions for an auxiliary nucleation problem formulated in the whole space and present nontrivial examples where the obtained bounds are tight.
\end{abstract}

\section{Introduction}

A classical problem of calculus of variation is to find global minimizers and identify in this way the most stable configurations (ground states). A solution of a much less studied problem of finding all local minimizers (metastable states) is obfuscated by the fact that local minima can be defined in different ways depending on the choice of the topology on the set of configurations. In this paper we consider two such topologies associated with weak and strong local minimizers. For each topology we pose the new problem of characterizing all minimally stable local minimizers.

In nonlinear elasticity reaching the associated marginally stable states usually means either structural or material failure [112, 29, 102, 7]. In this paper we do not address structural instabilities involving buckling, barreling, microstructure collapse, etc. which are all global phenomena [57, 103, 75, 44, 50, 106, 18, 19]. Instead, we focus on material instabilities that manifest themselves at a point and are geometry and inhomogeneity independent. In mechanical terms such instabilities may lead to the nucleation of cracks, cavities, second phase inclusions, dislocation loops and shear bands [58, 11, 13, 82, 76, 84]. Reaching local marginal stability thresholds is often associated with the transition from statics to dynamics, moreover, marginally stable states continue to be crucial in quasi-static flows. In particular, marginally stable states play a dominant role in self organization towards criticality as observed in plasticity, friction, fracture, martensitic phase transitions and damage $[124,4,101,100]$.

In applications it is important to identify states with disappearing reserve of stability in order to predict large and sometimes catastrophic changes associated with ensuing instabilities. In this paper we formulate the problem of finding the "unsafe loads" in the sense of the $W^{1, \infty}$ norm topology and the $W^{1, \infty}$ sequential weak-* topology. Our choice of topologies is by no means exhaustive, for instance, it excludes cavitation, while capturing phase transitions. For our two chosen topologies we propose a systematic procedure allowing one to bound marginally unsafe loads by solving an auxiliary problem formulated either for a system of partial differential equations or an algebraic system.

The simplest examples of material instabilities that can be linked to the two topologies studied in this paper can be found in fluids where the loss of weak local stability is associated with spinodal decomposition, and the corresponding marginally stable states lie on the spinodal [74], while the loss of strong local stability is associated with nucleation, and the corresponding stability threshold is called the binodal [115]. In this paper we study a far reaching generalization of these physical concepts in the context of calculus of variation. 
In the absence of better choices we continue using the physical terms spinodal and binodal as the indicators of weak and strong marginally stable states, respectively.

In mathematical literature the notion of weak global stability is associated with non-negativity of second variation $[14,104,45]$ and the full understanding of this concept in the scalar case was already achieved in the classical work of Jacobi who characterized bifurcation points of the Euler-Lagrange equations for second variation $[87,43,45]$. The general criterion of weak local stability is given by the Legendre-Hadamard condition whose relation to ellipticity loss of the Euler-Lagrange equations and associated bifurcations has been thoroughly studied $[116,8,35,30,64,9,93]$. In the context of nonlinear elasticity, the locus of weak marginally stable deformation gradients can often be fully characterized analytically $[63,125,31]$.

The mathematical notion of strong local, or material stability is expressed by the quasiconvexity condition $[86,10,102,13,32]$. Unlike the Legendre-Hadamard condition, this constraint is non-local and is much harder to explicate [69]. The quasi-convexification is known explicitly only in a few very special cases [61, 68,67 , $6,95,32]$ and our goal is to solve a simpler problem of computing the elastic binodal, without getting into a task of relaxing a non-quasiconvex energy. The complete solution of the binodal problem remains elusive even though we succeed in developing a systematic method of constructing a set of bounds distinguishing (strongly) unstable states from the stable ones.

It is appropriate to mention here that both weak and strong versions of material stability have nontrivial heterogeneous versions when the point of interest is located on the Neumann part of the external boundary $[2,3,20,14,105,102,85]$ or on an internal point of inhomogeneity [33, 75, 56]. Although the associated instabilities play important role in applications, e.g. [110, 59], we leave this interesting subject outside the scope of the present paper.

The main tool of our analysis is the notion of stability with respect to nucleation which we formulate, building on some earlier insights [78, 77, 97, 98, 99,61,60,41,42,40], in terms of solvability conditions for an auxiliary problem in all physical space. The infinity of the domain reflects the fact that nucleation is a manifestation of a local instability. We show that the nucleation problem can be formulated in different equivalent ways depending on the assumed behavior of the nucleus at infinity. We observe that, the seemingly natural requirement of attenuation at infinity is inadequate for capturing non-compact nuclei. Therefore we raise the problem of extending the space of test functions in order to obtain the broadest possible notion of the energy-neutral nucleus. Here, in contrast with the classical bifurcation theory, which is fully adequate in the case of the spinodal $[112,93,102]$, the implied generalized bifurcation problem cannot be understood by linearization. We refer to the similar development in the theory of shape optimization where non convex functionals arise naturally and our nucleation problem can be linked to the computation of 'topological or Hadamard derivatives' $[107,5]$.

More specifically, we show that by probing a homogeneous configuration with the test functions from sufficiently large spaces one can indeed obtain a partial characterization of the binodal (see earlier related work reviewed in $[81,24,25,26]$ ). How tight are the ensuing bounds depends on specifics of the non-convexity of the energy density function. While, it is unlikely that for a general energy density function there exists even a single point on the binodal which can be identified exactly by solving the classical nucleation problem for a PDE system, this is often a simple way to obtain surprisingly effective bounds. To supplement the PDEbased bounds, one can consider gradient Young measures of the sequences of test functions converging only weakly $[121,122,12,15,94,111]$. Finding the optimal Young measures in the general case is hardly possible, however, simple algebraic bounds on the binodal can be obtained by energy minimization with respect to a subclass of Young measures represented by laminates. Even though such results are only partial from the theoretical view point, the resulting bounds may be very useful in applications, where one has no hope of computing the explicit quasiconvex envelopes. A nontrivial example presented in this paper shows that in some cases the PDE-based methods combined with the laminate-based methods allow one to locate the entire elastic binodal.

The paper is organized as follows. In Section 2 we introduce the concepts of elastic spinodal and elastic binodal as the boundaries of the larger sets on which certain variational functionals are non-negative. To identify these boundaries one needs to solve a bifurcation problem in the case of the spinodal and a nucleation problem in the case of the binodal. The precise formulations of these problems depend on the functional and 
the chosen space of test functions. Different formulations defining the same critical sets form an equivalence class. In Section 3 we present several examples of equivalent formulations of the bifurcation and nucleation problems and propose the existence and the computability of solutions as possible selection criteria. In Section 4 we obtain an explicit characterization of the spinodal and binodal sets for some classes of test functions. In particular, we study the case when the binodal is detectable by solving a system of PDEs complemented with additional conditions allowing one to locate the singularities. A case study for an important class of bi-quadratic energies with two isotropic wells is presented in Section 5. Here we treat the case of arbitrary space dimensions and make the nucleation-based bounds on the binodal fully explicit. The last Section 6 contains our conclusions.

In this paper we will use subscripts like $W_{\boldsymbol{F}}(\boldsymbol{F})$ or $W_{\boldsymbol{F F}}(\boldsymbol{F})$ to denote the array of partial derivatives $\partial W / \partial F_{i \alpha}$ or $\partial^{2} W / \partial F_{i \alpha} \partial F_{j \beta}$ with respect to field variables $\boldsymbol{F}$ and we use $\nabla \boldsymbol{y}(\boldsymbol{x})$ to denote the matrix $(\nabla \boldsymbol{y})_{i \alpha}=$ $\partial y_{i} / \partial x_{\alpha}$ of partial derivatives. We also use the inner product notation $(\boldsymbol{a}, \boldsymbol{b})$ to denote the dot product of two vectors or a Frobenius inner product $\operatorname{Tr}\left(\boldsymbol{A} \boldsymbol{B}^{T}\right)$ of two matrices. The notation $\boldsymbol{A} \boldsymbol{x}$ denotes the matrix-vector multiplication and $W_{\boldsymbol{F F}}(\boldsymbol{F}) \boldsymbol{H}$ denotes the action of the operator $W_{\boldsymbol{F} F}$ on the space $\mathbb{M}$ of $m \times d$ matrices:

$$
\left(W_{\boldsymbol{F} F}(\boldsymbol{F}) \boldsymbol{H}\right)_{i \alpha}=\sum_{j=1}^{m} \sum_{\beta=1}^{d} \frac{\partial^{2} W}{\partial F_{i \alpha} \partial F_{j \beta}} H_{j \beta} .
$$

\section{Spinodal and binodal}

Consider a rather general variational functional used in non-linear elasticity theory

$$
E(\boldsymbol{y})=\int_{\Omega} W(\nabla \boldsymbol{y}(\boldsymbol{x})) d \boldsymbol{x}-\int_{\partial \Omega_{N}}(\boldsymbol{t}(\boldsymbol{x}), \boldsymbol{y}) d S(\boldsymbol{x}),
$$

where $\Omega$ is a smooth open and bounded domain in $\mathbb{R}^{d}$, and $\partial \Omega_{N}$ is the Neumann part of the boundary. We assume that the values of $\boldsymbol{y}(\boldsymbol{x})$ are prescribed on the Dirichlet part $\partial \Omega_{D}=\partial \Omega \backslash \partial \Omega_{N}$. The energy density $W$ is a continuous and bounded from below function on the space $\mathbb{M}$ of all $m \times d$ matrices. Further regularity assumptions will be stated below. We observe that in general it is possible to absorb the boundary term into the volume integral by replacing the energy density with appropriate more general Lagrangian

$$
E(\boldsymbol{y})=\int_{\Omega} L(\boldsymbol{x}, \boldsymbol{y}(\boldsymbol{x}) \nabla \boldsymbol{y}(\boldsymbol{x})) d \boldsymbol{x} .
$$

To formulate our two notions of local stability (or metastability) we define $\operatorname{Var}=\left\{\boldsymbol{u} \in C^{1}\left(\bar{\Omega} ; \mathbb{R}^{m}\right)\right.$ : $\left.\boldsymbol{u}(\boldsymbol{x})=\mathbf{0}, \boldsymbol{x} \in \partial \Omega_{D}\right\}$. The weakly and strongly stable states are defined as follows.

Definition 2.1. A sequence $\boldsymbol{u}_{n} \in \operatorname{Var}$ is called an admissible weak variation if $\left\|\boldsymbol{u}_{n}\right\|_{C^{1}(\Omega)} \rightarrow 0$, as $n \rightarrow \infty$. Correspondingly, we say that $\boldsymbol{y}(\boldsymbol{x})$ is weakly stable, if for all admissible weak variations $\boldsymbol{u}_{n}$ we have $E\left(\boldsymbol{y}+\boldsymbol{u}_{n}\right) \geq E(\boldsymbol{y})$ when $n$ is sufficiently large.

Definition 2.2. A sequence $\boldsymbol{s}_{n} \in \operatorname{Var}$ is called an admissible strong variation if $\left\|\boldsymbol{s}_{n}\right\|_{C(\Omega)} \rightarrow 0$, as $n \rightarrow \infty$. Correspondingly, we say that $\boldsymbol{y}(\boldsymbol{x})$ is stable, if for all admissible strong variations $\boldsymbol{s}_{n}$ we have $E\left(\boldsymbol{y}+\boldsymbol{s}_{n}\right) \geq E(\boldsymbol{y})$ when $n$ is sufficiently large.

Suppose now that we are testing stability of a given configuration $\boldsymbol{y} \in C^{1}\left(\bar{\Omega} ; \mathbb{R}^{m}\right)$. We always assume that the energy density $L(\boldsymbol{x}, \boldsymbol{y}, \boldsymbol{F})$ is of class $C^{2}$ on the extended graph $\{(\boldsymbol{x}, \boldsymbol{y}(\boldsymbol{x}), \nabla \boldsymbol{y}(\boldsymbol{x})): \boldsymbol{x} \in \bar{\Omega}\}$ of $\boldsymbol{y}(\boldsymbol{x})$. Consider a general (weak or strong) admissible variation $\left\{\boldsymbol{g}_{\epsilon}\right\} \subset$ Var. We now expand the energy increment

$$
\Delta E\left(\boldsymbol{g}_{\epsilon}\right)=E\left(\boldsymbol{y}+\boldsymbol{g}_{\epsilon}\right)-E(\boldsymbol{y})=\delta E\left(\boldsymbol{g}_{\epsilon}\right)+\delta^{2} E\left(\boldsymbol{g}_{\epsilon}\right) .
$$

where

$$
\delta E\left(\boldsymbol{g}_{\epsilon}\right)=\int_{\Omega}\left\{\left(L_{\boldsymbol{F}}, \nabla \boldsymbol{g}_{\epsilon}\right)+\left(L_{\boldsymbol{y}}, \boldsymbol{g}_{\epsilon}\right)\right\} d \boldsymbol{x}
$$


The second term

$$
\delta^{2} E\left(\boldsymbol{g}_{\epsilon}\right)=\int_{\Omega} L^{\star}\left(\boldsymbol{x}, \boldsymbol{g}_{\epsilon}, \nabla \boldsymbol{g}_{\epsilon}\right) d \boldsymbol{x}
$$

where

$$
L^{\star}(\boldsymbol{x}, \boldsymbol{u}, \boldsymbol{H})=L(\boldsymbol{x}, \boldsymbol{y}(\boldsymbol{x})+\boldsymbol{u}, \nabla \boldsymbol{y}(\boldsymbol{x})+\boldsymbol{H})-L(\boldsymbol{x})-\left(L_{\boldsymbol{F}}(\boldsymbol{x}), \boldsymbol{H}\right)-\left(L_{\boldsymbol{y}}(\boldsymbol{x}), \boldsymbol{u}\right),
$$

can be formally interpreted as the "generalized second variation." Indeed, for the weak variations of the form

$$
\boldsymbol{g}_{\epsilon}=\epsilon \boldsymbol{u}, \quad \boldsymbol{u} \in C^{1}\left(\bar{\Omega} ; \mathbb{R}^{m}\right) \cap \operatorname{Var},
$$

we have

$$
\delta^{2} E\left(\boldsymbol{g}_{\epsilon}\right)=\frac{\epsilon^{2}}{2} \int_{\Omega}\left\{\left(L_{\boldsymbol{F} F} \nabla \boldsymbol{u}, \nabla \boldsymbol{u}\right)+2\left(L_{\boldsymbol{y} \boldsymbol{F}} \nabla \boldsymbol{u}, \boldsymbol{u}\right)+\left(L_{\boldsymbol{y} \boldsymbol{y}} \boldsymbol{u}, \boldsymbol{u}\right)\right\} d \boldsymbol{x}+o\left(\epsilon^{2}\right) .
$$

Since the linear term $\delta E\left(\boldsymbol{g}_{\epsilon}\right)$ in the expansion (2.3) vanishes due to the Euler-Lagrange equation, the requirement $\Delta E\left(\boldsymbol{g}_{\epsilon}\right) \geq 0$ implies, for the class of special weak variations (2.4), the non-negativity of the classical second variation

$$
\int_{\Omega}\left\{\left(L_{\boldsymbol{F} F} \nabla \boldsymbol{u}, \nabla \boldsymbol{u}\right)+2\left(L_{\boldsymbol{y} \boldsymbol{F}} \nabla \boldsymbol{u}, \boldsymbol{u}\right)+\left(L_{\boldsymbol{y} \boldsymbol{y}} \boldsymbol{u}, \boldsymbol{u}\right)\right\} d \boldsymbol{x} \geq 0
$$

for all $\boldsymbol{u} \in$ Var. The condition of non-negativity of the generalized second variation condition produces a different result if the general variation is taken from the special class of strong variations

$$
\boldsymbol{g}_{\eta}=\eta \boldsymbol{\phi}\left(\left(\boldsymbol{x}-\boldsymbol{x}_{0}\right) / \eta, \quad \boldsymbol{x}_{0} \in \Omega, \quad \boldsymbol{\phi} \in C_{0}^{1}\left(B_{1} ; \mathbb{R}^{m}\right) .\right.
$$

If $\nabla \boldsymbol{y}(\boldsymbol{x})$ is continuous at $\boldsymbol{x}_{0}$, then the generalized second variation has the form

$$
\delta^{2} E\left(\boldsymbol{g}_{\eta}\right)=\eta^{d} \int_{B_{1}} W^{\circ}\left(\nabla \boldsymbol{y}\left(\boldsymbol{x}_{0}\right), \nabla \boldsymbol{\phi}\right) d \boldsymbol{z}+o\left(\eta^{d}\right),
$$

where

$$
W^{\circ}(\boldsymbol{F}, \boldsymbol{H})=\mathcal{E}_{L}\left(\boldsymbol{x}_{0}, \boldsymbol{y}\left(\boldsymbol{x}_{0}\right), \boldsymbol{F}, \boldsymbol{F}+\boldsymbol{H}\right),
$$

and

$$
\mathcal{E}_{L}\left(\boldsymbol{x}, \boldsymbol{y}, \boldsymbol{F}, \boldsymbol{F}^{\prime}\right)=L\left(\boldsymbol{x}, \boldsymbol{y}, \boldsymbol{F}^{\prime}\right)-L(\boldsymbol{x}, \boldsymbol{y}, \boldsymbol{F})-\left(L_{\boldsymbol{F}}(\boldsymbol{x}, \boldsymbol{y}, \boldsymbol{F}), \boldsymbol{F}^{\prime}-\boldsymbol{F}\right)
$$

is the classical Weierstrass excess function for the Lagrangian $L[123,45]$. We see that $W^{\circ}(\boldsymbol{F}, \boldsymbol{H})$ can be expressed entirely in terms of the localized version of the Lagrangian

$$
W(\boldsymbol{F})=L\left(\boldsymbol{x}_{0}, \boldsymbol{y}\left(\boldsymbol{x}_{0}\right), \boldsymbol{F}\right),
$$

where the dependence on $\boldsymbol{x}_{0} \in \Omega$ is suppressed in the notation. We note that if the Lagrangian $L(\boldsymbol{x}, \boldsymbol{y}, \boldsymbol{F})$ comes from the energy of the form (2.1) then our definition of $W(\boldsymbol{F})$ differs from the original $W(\boldsymbol{F})$ by at most a linear term, which does not affect any of the subsequent equations.

The requirement $\Delta E\left(\boldsymbol{g}_{\eta}\right) \geq 0$ for the class of special strong variations (2.6) is equivalent to the the quasiconvexity at $\nabla \boldsymbol{y}\left(\boldsymbol{x}_{0}\right),[86,10]$ :

$$
\int_{B_{1}} W^{\circ}\left(\nabla \boldsymbol{y}\left(\boldsymbol{x}_{0}\right), \nabla \boldsymbol{\phi}(\boldsymbol{z})\right) d \boldsymbol{z} \geq 0
$$

for all $\phi \in C_{0}^{1}\left(B_{1} ; \mathbb{R}^{m}\right)$. Notice that the infinitesimal perturbation (2.6) at a point $\boldsymbol{x}_{0} \in \Omega$ is transformed by rescaling (zooming in) into a finite perturbation prescribed on the unit ball. It is well known that the condition (2.9) does not depend on the support of $\boldsymbol{\phi}(\boldsymbol{x})$ and the unit ball $B_{1}$ can be replaced by any subset of $\mathbb{R}^{d}$, as long as its boundary has zero $d$-volume. The smoothness of $\phi(x)$ is also not important, in particular, the condition (2.9) would be unchanged if we require that $\phi$ be of class $C^{\infty}$, or if we allow $\phi$ to be merely Lipschitz continuous. 
The removal of the linear term in (2.3) is natural since we consider stability of an equilibrium state and this step is straightforward in the case of weak local minima. For strong local minima, the removal of the linear term, dating back to Weierstrass, is also useful because finite perturbations in a small domain create small perturbations outside this domain, and the latter become invisible if the linear part of the functional is removed.

We emphasize that while the quasiconvexity condition (2.9) is domain-local, i.e. it depends only on the behavior of the deformation $\boldsymbol{y}(\boldsymbol{x})$ in any neighborhood of the point $\boldsymbol{x}_{0}$, the second variation condition (2.5) is domain-global. The two conditions, however, have a non-trivial intersection that can be achieved either by performing the "localization" $\boldsymbol{u}(\boldsymbol{x}) \mapsto \eta \boldsymbol{v}\left(\left(\boldsymbol{x}-\boldsymbol{x}_{0}\right) / \eta\right)$ in $(2.5)$ with $\boldsymbol{v} \in C_{0}^{1}\left(B_{1} ; \mathbb{R}^{m}\right)$ or by the "weakening" $\phi(z) \mapsto \epsilon \boldsymbol{v}(\boldsymbol{z})$, in (2.9) with $\boldsymbol{v} \in C_{0}^{1}\left(B_{1} ; \mathbb{R}^{m}\right)$. Independently of whether we take a limit $\epsilon \rightarrow 0$ in (2.9) or the limit $\eta \rightarrow 0$ in (2.5) we obtain

$$
\int_{B_{1}}\left(W_{\boldsymbol{F} F}\left(\nabla \boldsymbol{y}\left(\boldsymbol{x}_{0}\right)\right) \nabla \boldsymbol{v}(\boldsymbol{z}), \nabla \boldsymbol{v}(\boldsymbol{z})\right) d \boldsymbol{z} \geq 0
$$

for all $\boldsymbol{v} \in C_{0}^{1}\left(B_{1} ; \mathbb{R}^{m}\right)$.

The necessary conditions (2.9) and (2.10) of strong and weak stability motivate the following definitions of spinodal and binodal.

Definition 2.3. The deformation gradient $\boldsymbol{F} \in \mathbb{M}$ is called weakly locally stable if

$$
\int_{B_{1}}\left(W_{\boldsymbol{F} \boldsymbol{F}}(\boldsymbol{F}) \nabla \boldsymbol{v}(\boldsymbol{z}), \nabla \boldsymbol{v}(\boldsymbol{z})\right) d \boldsymbol{z} \geq 0
$$

for all $\boldsymbol{v} \in C_{0}^{1}\left(B_{1} ; \mathbb{R}^{m}\right)$. The set

$$
\mathfrak{S}=\{\boldsymbol{F} \in \mathbb{M}: \boldsymbol{F} \text { is not weakly locally stable }\}
$$

is called the spinodal region. The boundary surface $\mathfrak{S p i n}=\partial \mathfrak{S}$ of the spinodal region is called the spinodal.

Definition 2.4. The deformation gradient $\boldsymbol{F} \in \mathbb{M}$ is called strongly locally stable if

$$
\int_{B_{1}} W^{\circ}(\boldsymbol{F}, \nabla \boldsymbol{\phi}(\boldsymbol{z})) d \boldsymbol{z} \geq 0
$$

for all $\phi \in C_{0}^{1}\left(B_{1} ; \mathbb{R}^{m}\right)$. The set

$$
\mathfrak{B}=\{\boldsymbol{F} \in \mathbb{M}: \boldsymbol{F} \text { is not strongly locally stable }\}
$$

is called the binodal region. The boundary surface $\mathfrak{B i n}=\partial \mathfrak{B}$ of the binodal region is called the binodal.

Our goal is to formulate conditions on $\boldsymbol{F}$ under which the inequalities (2.12) and (2.11) become violated. The spinodal and the binodal regions can be characterized in terms of the parametric variational inequalities

$$
\begin{gathered}
\mathfrak{S}=\left\{\boldsymbol{F} \in \mathbb{M}: \inf _{\boldsymbol{\phi} \in C_{0}^{1}\left(B_{1} ; \mathbb{R}^{m}\right)} \int_{B_{1}}\left(W_{\boldsymbol{F} \boldsymbol{F}}(\boldsymbol{F}) \nabla \boldsymbol{v}(\boldsymbol{z}), \nabla \boldsymbol{v}(\boldsymbol{z})\right) d \boldsymbol{z}<0\right\}, \\
\mathfrak{B}=\left\{\boldsymbol{F} \in \mathbb{M}: \inf _{\boldsymbol{\phi} \in C_{0}^{1}\left(B_{1} ; \mathbb{R}^{m}\right)} \int_{B_{1}} W^{\circ}(\boldsymbol{F}, \nabla \boldsymbol{\phi}(\boldsymbol{z})) d \boldsymbol{z}<0\right\} .
\end{gathered}
$$

In view of the nonexistence issues neither (2.14) nor (2.15) allows one to characterize the corresponding sets directly in the general case. For the weak local stability in the vectorial case $d>1$ the absence of the nontrivial solutions for the corresponding Euler-Lagrange equation does not imply stability because the boundary conditions are too restrictive. We therefore extend the space of admissible test functions by eliminating all unnecessary smoothness and growth conditions. While similar extension of the space of admissible functions 
will not solve the non-attainment problem in the case of the general binodal, in many specific examples even a limited extension allows one to obtain a fairly complete picture.

More precisely, we use a crucial observation that in order to characterize the binodal we do not need to know the value of the infimum in (2.15) but only its sign. In this sense our problem is less difficult than the problem of computing the quasiconvex envelope of $W(\boldsymbol{F})$. Most importantly, we can expect that various functionals may fully characterize the binodal via their signs and other parametrized variational inequalities equivalent to (2.15) can be used instead. This observation suggests the following definitions.

Definition 2.5. Let $\mathcal{F} \subset W^{1, \infty}\left(\mathbb{R}^{d} ; \mathbb{R}^{m}\right)$ and let $I(\boldsymbol{F}, \phi)$ be the functional on $\mathcal{F}$. We say that the pair $(I, \mathcal{F})$ bounds the spinodal (binodal) if for every $\boldsymbol{F} \notin \mathfrak{S}(\boldsymbol{F} \notin \mathfrak{B})$

$$
\inf _{\boldsymbol{\phi} \in \mathcal{F}} I(\boldsymbol{F}, \boldsymbol{\phi}) \geq 0 \text {. }
$$

We say that the pair $(I, \mathcal{F})$ characterizes the spinodal (binodal) if, in addition to (2.16), for every $\boldsymbol{F} \in \mathfrak{S}$ $(\boldsymbol{F} \in \mathfrak{B})$

$$
\inf _{\phi \in \mathcal{F}} I(\boldsymbol{F}, \boldsymbol{\phi})<0 .
$$

We already know that the pair

$$
\mathcal{F}=C_{0}^{1}\left(B_{1} ; \mathbb{R}^{m}\right), \quad I(\boldsymbol{F}, \boldsymbol{\phi})=\int_{B_{1}}\left(W_{\boldsymbol{F} \boldsymbol{F}}(\boldsymbol{F}) \nabla \boldsymbol{v}(\boldsymbol{z}), \nabla \boldsymbol{v}(\boldsymbol{z})\right) d \boldsymbol{z}
$$

characterizes the spinodal, while the pair

$$
\mathcal{F}=C_{0}^{1}\left(B_{1} ; \mathbb{R}^{m}\right), \quad I(\boldsymbol{F}, \boldsymbol{\phi})=\int_{B_{1}} W^{\circ}(\boldsymbol{F}, \nabla \boldsymbol{\phi}(\boldsymbol{z})) d \boldsymbol{z}
$$

characterizes the binodal. Another well-known example of the binodal characterizing pair is $\left(\mathcal{Y}_{c}^{0}, \Lambda^{\circ}\right)$, where $\mathcal{Y}_{c}^{0}$ is the space of homogeneous compactly supported gradient Young measures with zero first moment and $\Lambda^{\circ}$ is a linear functional on $\mathcal{Y}_{c}^{0}$ defined by

$$
\Lambda^{\circ}(\nu)=\int_{\mathbb{M}} W^{\circ}(\boldsymbol{F}+\boldsymbol{H}) d \nu(\boldsymbol{H}), \quad \nu \in \mathcal{Y}_{c}^{0} .
$$

Since none of these characterizations of the binodal is practical, our goal will be to present other pairs $(\mathcal{F}, I)$ that characterize the binodal. In particular, we are interested in finding the spaces $\mathcal{F}$ that allow one to characterize the binodal in terms of computable solutions of a system of PDEs. Observe that the notions of pairs characterizing and bounding the binodal may go beyond a simple extension of a function space. For example, nucleation of a precipitate containing martensitic twins microstructure in a shape memory alloy $[97,98,99,126,66,65]$, suggests that the set $\mathcal{F}$ may contain parametrized families of Young measures, while the functional $I$ in the pair $(\mathcal{F}, I)$ will then be derived as a limit of the original functional on the sequences generating the Young measures.

\section{Equivalent problems}

In this section we present several examples of spinodal-characterizing, binodal-characterizing and binodalbounding pairs that are different from those given in (2.14) and (2.15) and are better suited for obtaining explicit constraints for the sets $\mathfrak{S p i n}$ and $\mathfrak{B i n}$.

\subsection{Spinodal}

In the case of spinodal, the functional in (2.11) is quadratic, and hence, it is natural to extend the space $C_{0}^{1}\left(B_{1}, \mathbb{R}^{m}\right)$ to the space

$$
\mathcal{S}_{0}=\left\{\boldsymbol{v} \in L_{\mathrm{loc}}^{2}\left(\mathbb{R}^{d} ; \mathbb{R}^{m}\right): \nabla \boldsymbol{v} \in L^{2}\left(\mathbb{R}^{d} ; \mathbb{M}\right)\right\}
$$


Then the pair $\left(\mathcal{S}_{0}, I_{0}\right)$ is spinodal-characterizing, where

$$
I_{0}(\boldsymbol{F}, \boldsymbol{\phi})=\int_{\mathbb{R}^{d}}\left(W_{\boldsymbol{F} \boldsymbol{F}}(\boldsymbol{F}) \nabla \boldsymbol{v}(\boldsymbol{z}), \nabla \boldsymbol{v}(\boldsymbol{z})\right) d \boldsymbol{z} \geq 0, \quad \boldsymbol{v} \in \mathcal{S}_{0} .
$$

Let us show that generically, when $d>1$ the associated Euler-Lagrange equation

$$
\nabla \cdot\left(W_{\boldsymbol{F} F}(\boldsymbol{F}) \nabla \boldsymbol{v}\right)=\mathbf{0}, \quad \boldsymbol{v} \in \mathcal{S}_{0}
$$

has no non-zero solutions. Indeed, taking the Fourier transform of $(3.3)$ we obtain $[116,112,102]$

$$
\boldsymbol{A}(\boldsymbol{m} ; \boldsymbol{F}) \widehat{\boldsymbol{v}}(\boldsymbol{m})=\mathbf{0},
$$

where the acoustic tensor $\boldsymbol{A}(\boldsymbol{m} ; \boldsymbol{F})$ at $\boldsymbol{F}$ is defined as the linear map on $\mathbb{R}^{m}$ given by

$$
\boldsymbol{a} \mapsto \boldsymbol{A}(\boldsymbol{m} ; \boldsymbol{F}) \boldsymbol{a}=\left(W_{\boldsymbol{F} \boldsymbol{F}}(\boldsymbol{F})(\boldsymbol{a} \otimes \boldsymbol{m})\right) \boldsymbol{m} .
$$

As we can see the $L^{2}$ function $\widehat{\boldsymbol{v}}(\boldsymbol{m}) \otimes \boldsymbol{m}$ must be supported on the union of rays $\mathbb{R} \boldsymbol{n}$, where $|\boldsymbol{n}|=1$ solves $\operatorname{det} \boldsymbol{A}(\boldsymbol{n} ; \boldsymbol{F})=0$. Generically, this union is a closed and nowhere dense subset of $\mathbb{R}^{d}$, when $d>1$. Hence $\widehat{\nabla \boldsymbol{v}}(\boldsymbol{m})=\mathbf{0}$ for a.e. $\boldsymbol{m} \in \mathbb{R}^{d}$, and the problem (3.3) has only trivial solutions in $\mathcal{S}_{0}$. The reason for nonexistence in (3.3) is that the eigenfunctions of the second order differential operator with constant coefficients are single Fourier modes $e^{i(\boldsymbol{n}, \boldsymbol{z})} \boldsymbol{a}$, where $\boldsymbol{a}$ is an eigenvector of the acoustic tensor and these eigenfunctions do not belong to $\mathcal{S}_{0}$.

The set of functions containing the eigen-modes of the linear operator (3.3) should be sufficient to characterize the spinodal. One possible choice is the set

$$
\mathcal{F}_{\mathfrak{S}}=\left\{\phi((\boldsymbol{z}, \boldsymbol{n})) \boldsymbol{a}: \phi \in H^{1}(\mathbb{R})\right\}
$$

of functions, whose distributional Fourier transform is supported on a single ray $\mathbb{R} \boldsymbol{n}$.

Observe that the functions from (3.5) decay at infinity only in one direction $\boldsymbol{n}$ and the quadratic functional in (3.2) is no longer defined. To fix this problem we can approximate functions (3.5) by a sequence of functions $\boldsymbol{v}_{\eta} \in \mathcal{S}_{0}$ and consider an equivalent functional

$$
I(\boldsymbol{F}, \boldsymbol{v})=\frac{\int_{\mathbb{R}^{d}}\left(W_{\boldsymbol{F} \boldsymbol{F}}(\boldsymbol{F}) \nabla \boldsymbol{v}, \nabla \boldsymbol{v}\right) d \boldsymbol{z}}{\int_{\mathbb{R}^{d}}|\nabla \boldsymbol{v}|^{2} d \boldsymbol{z}}, \quad \boldsymbol{v} \in \mathcal{S}_{0} .
$$

Indeed, it is obvious that $I(\boldsymbol{F}, \boldsymbol{v}) \geq 0$ for all $\boldsymbol{v} \in \mathcal{S}_{0}$ if and only if (3.2) holds. Let $\rho(\boldsymbol{x})$ be a smooth compactly supported function then

$$
\lim _{\eta \rightarrow 0} I(\boldsymbol{F}, \rho(\eta \boldsymbol{z}) \phi((\boldsymbol{z}, \boldsymbol{n})) \boldsymbol{a})=(\boldsymbol{A}(\boldsymbol{n} ; \boldsymbol{F}) \boldsymbol{a}, \boldsymbol{a}) .
$$

This formula follows from the relation

$$
\lim _{\eta \rightarrow 0} \int_{\mathbb{R}^{d}}\left(W_{\boldsymbol{F} F}(\boldsymbol{F}) \nabla \boldsymbol{v}_{\eta}, \nabla \boldsymbol{v}_{\eta}\right) d \boldsymbol{z}=(\boldsymbol{A}(\boldsymbol{n} ; \boldsymbol{F}) \boldsymbol{a}, \boldsymbol{a})\left(\int_{\mathbb{R}} \phi^{\prime}(t)^{2} d t\right)\left(\int_{(\mathbb{R} \boldsymbol{n})^{\perp}} \rho(\boldsymbol{u})^{2} d S(\boldsymbol{u})\right),
$$

where

$$
\boldsymbol{v}_{\eta}(\boldsymbol{z})=\eta^{\frac{d-1}{2}} \rho(\eta \boldsymbol{z}) \phi((\boldsymbol{z}, \boldsymbol{n})) \boldsymbol{a} .
$$

It is now easy to show that the pair $\left(\mathcal{F}_{\mathfrak{S}}, I_{\mathfrak{S}}\right)$ with

$$
I_{\mathfrak{S}}(\boldsymbol{F}, \phi((\boldsymbol{z}, \boldsymbol{n})) \boldsymbol{a})=(\boldsymbol{A}(\boldsymbol{n} ; \boldsymbol{F}) \boldsymbol{a}, \boldsymbol{a}) .
$$

characterizes the spinodal. The Plancherel's identity applied to (3.2) implies

$$
\int_{\mathbb{R}^{d}}(\boldsymbol{A}(\boldsymbol{m} ; \boldsymbol{F}) \widehat{\boldsymbol{v}}(\boldsymbol{m}), \widehat{\boldsymbol{v}}(\boldsymbol{m})) d \boldsymbol{m} \geq 0 .
$$

It is obvious now, that if the acoustic tensor $\boldsymbol{A}(\boldsymbol{n} ; \boldsymbol{F}) \geq 0$ in the sense of quadratic forms for all $\boldsymbol{n} \in \mathbb{S}^{d-1}$, then (3.9) and hence (3.2) holds. Conversely, (3.7) shows that (3.2) implies non-negativity of the acoustic tensor. 
Remark 3.1. Due to the homogeneity of the functional $I_{\mathfrak{S}}$ we can also write

$$
\inf _{\boldsymbol{\phi} \in \mathcal{F}_{\mathfrak{S}}} I_{\mathfrak{S}}(\boldsymbol{F}, \boldsymbol{\phi})=-\operatorname{Ind}_{\mathfrak{S}^{c}}(\boldsymbol{F})
$$

where $\operatorname{Ind}_{\mathfrak{S}^{c}}(\boldsymbol{F})$ is the indicator function ${ }^{1}$ of the complement to the spinodal region $\mathfrak{S}$.

\subsection{Binodal}

Here we proceed in parallel with the analysis for the spinodal. We begin by extending the space of admissible test functions from $C_{0}^{1}\left(B_{1} ; \mathbb{R}^{m}\right)$ to the space

$$
\mathcal{S}=\left\{\boldsymbol{\phi} \in W^{1, \infty}\left(\mathbb{R}^{d} ; \mathbb{R}^{m}\right): \nabla \phi \in L^{2}\left(\mathbb{R}^{d} ; \mathbb{M}\right)\right\},
$$

for which the integral

$$
I^{\circ}(\boldsymbol{F}, \boldsymbol{\phi})=\int_{\mathbb{R}^{d}} W^{\circ}(\boldsymbol{F}, \nabla \phi(\boldsymbol{z})) d \boldsymbol{z}
$$

is convergent. We emphasize the additional assumption of uniform boundedness of $\phi$ and $\nabla \phi$ in the definition of the space $\mathcal{S}$. From a technical standpoint the assumption $\phi \in \mathcal{S}_{0}$ is insufficient to ensure convergence of the integral in (3.12).

Interestingly, the phenomenon of cavitation [11, 109], which is outside the scope of this paper, can be interpreted as existence of unbounded Sobolev test fields $\phi \in W^{1, p}\left(\mathbb{R}^{d} ; \mathbb{R}^{m}\right)$ that in the hard device can lower the energy of a homogeneous state, which is not in the binodal region. Thus, examples in $[88,96]$ feature cavitation for globally polyconvex energies, whose binodal regions are empty sets.

TheOREM 3.2. The pair $\left(\mathcal{S}, I^{\circ}\right)$ characterizes the binodal.

Proof. If the inequality (2.12) fails for some $\phi \in C_{0}^{\infty}\left(B_{1} ; \mathbb{R}^{m}\right)$ then the inequality (3.12) fails, since $\phi \in \mathcal{S}$, if extended by zero outside of $B_{1}$.

First we prove (3.12), assuming that (2.12) holds. For each $R>0$ let $\eta_{R} \in C_{0}^{\infty}\left(B_{2 R}\right)$ be a cut-off function such that $\eta_{R}$ takes values between 0 and $1, \eta_{R}(\boldsymbol{x})=1$ for all $\boldsymbol{x} \in B_{R}$ and $\left|\nabla \eta_{R}(\boldsymbol{x})\right| \leq C / R$ for all $\boldsymbol{x} \in \mathbb{R}^{d}$ with constant $C$ independent of $R$. We extend $\eta_{R}(\boldsymbol{x})$ by zero to the complement of $B_{2 R}$. The theorem will be proved if we show that for each $\phi \in \mathcal{S}$ there exists a constant ${ }^{2} c \in \mathbb{R}^{m}$ and a sequence $R_{k} \rightarrow \infty$, such that

$$
\lim _{k \rightarrow \infty} \int_{\mathbb{R}^{d}} W^{\circ}\left(\boldsymbol{F}, \nabla\left(\eta_{R_{k}}(\boldsymbol{\phi}-\boldsymbol{c})\right) d \boldsymbol{z}=\int_{\mathbb{R}^{d}} W^{\circ}(\boldsymbol{F}, \nabla \boldsymbol{\phi}) d \boldsymbol{z},\right.
$$

Indeed, if $\boldsymbol{F}$ satisfies (2.12) then

$$
\int_{\mathbb{R}^{d}} W^{\circ}\left(\boldsymbol{F}, \nabla\left(\eta_{R_{k}}(\boldsymbol{\phi}-\boldsymbol{c})\right)\right) d \boldsymbol{z}=\int_{B_{2_{R_{k}}}} W^{\circ}\left(\boldsymbol{F}, \nabla\left(\eta_{R_{k}}(\boldsymbol{\phi}-\boldsymbol{c})\right)\right) d \boldsymbol{z} \geq 0
$$

for all $k \in \mathbb{N}$, and hence the relation (3.13) implies (3.12).

To prove (3.13) we use the inequality

$$
\left|W^{\circ}(\boldsymbol{F}, \boldsymbol{G}+\boldsymbol{H})-W^{\circ}(\boldsymbol{F}, \boldsymbol{G})\right| \leq C\left(|\boldsymbol{G}||\boldsymbol{H}|+|\boldsymbol{H}|^{2}\right)
$$

that holds for all $|\boldsymbol{G}| \leq M$ and $|\boldsymbol{H}| \leq M$, where the constant $C$ depends on $M, \boldsymbol{F}$ and $W$. Taking $M=\|\phi\|_{1, \infty}$, we have

$$
\left|W^{\circ}\left(\boldsymbol{F}, \nabla\left(\eta_{R}(\boldsymbol{\phi}-\boldsymbol{c})\right)\right)-W^{\circ}\left(\boldsymbol{F}, \eta_{R} \nabla \boldsymbol{\phi}\right)\right| \leq C\left(\frac{1}{R}|\boldsymbol{\phi}-\boldsymbol{c}||\nabla \boldsymbol{\phi}|+\frac{1}{R^{2}}|\boldsymbol{\phi}-\boldsymbol{c}|^{2}\right),
$$

\footnotetext{
${ }^{1}$ The indicator function of a set equals to zero on the set and $+\infty$ on its complement.

${ }^{2}$ When $d \geq 3$ there is a canonical choice of the constant $c$, such that $\phi-c \in L^{2 d / d-2}\left(\mathbb{R}^{d} ; \mathbb{R}^{m}\right)$ (see Theorem A.1). When $d=1$ the choice of the constant is irrelevant. However, when $d=2$ there is no canonical choice of the constant $\boldsymbol{c}$, which can not be chosen arbitrarily.
} 
where the constant $C$ is independent of $\boldsymbol{x}$ and $R$. Lemma 3.3 below implies there exists a constant $\boldsymbol{c} \in \mathbb{R}^{m}$ and a subsequence $R_{k}$, such that

$$
\lim _{k \rightarrow \infty} \int_{\mathbb{R}^{d}} W^{\circ}\left(\boldsymbol{F}, \nabla\left(\eta_{R_{k}}(\boldsymbol{\phi}-\boldsymbol{c})\right) d \boldsymbol{z}=\lim _{k \rightarrow \infty} \int_{\mathbb{R}^{d}} W^{\circ}\left(\boldsymbol{F}, \eta_{R_{k}} \nabla \boldsymbol{\phi}\right) d \boldsymbol{z} .\right.
$$

The inequality $W^{\circ}(\boldsymbol{F}, \boldsymbol{H}) \leq C|\boldsymbol{H}|^{2}$ holds for all $|\boldsymbol{H}| \leq M$, where the constant $C$ depends $M$. Using this inequality with $M=\|\phi\|_{1, \infty}$ permits the application of the Lebesgue dominated convergence theorem, resulting in (3.13).

Now we formulate the Lemma which we needed in Theorem 3.2.

Lemma 3.3. For any $\phi \in \mathcal{S}$ there exists a constant $\boldsymbol{c} \in \mathbb{R}^{m}$, such that

$$
\varliminf_{R \rightarrow \infty} \int_{A_{R}}\left\{\frac{1}{R}|\boldsymbol{\phi}-\boldsymbol{c}||\nabla \boldsymbol{\phi}|+\frac{1}{R^{2}}|\boldsymbol{\phi}-\boldsymbol{c}|^{2}\right\} d \boldsymbol{x}=0
$$

where $A_{R}=B_{2 R} \backslash B_{R}$.

The proof of the lemma is in Appendix A.

Remark 3.4. In the statement of Lemma 3.3 the liminf can be replaced by limit as $R \rightarrow \infty$ (as we can see from the proof), except when $d=2$. When $d=2$, the use of liminf is essential. Indeed, let $\phi(\boldsymbol{x})=u(|\boldsymbol{x}|)$, where

$$
u(r)=2 \sin (\ln \ln r)+\frac{\cos (\ln \ln r)}{\ln r}, \quad r \geq e .
$$

We compute

$$
\langle\phi\rangle_{A_{R}}=\frac{2}{3 R^{2}} \int_{R}^{2 R} r u(r) d r=\left.\frac{2}{3 R^{2}}\left(r^{2} \sin (\ln \ln r)\right)\right|_{R} ^{2 R}=2 \sin (\ln \ln R)+o(1),
$$

as $R \rightarrow \infty$. We see that $\langle\phi\rangle_{A_{R}}$ has no limit as $R \rightarrow \infty$ and that we can choose any $c \in[-2,2]$ so that there is a sequence $R_{k}$ for which $\langle\phi-c\rangle_{A_{R_{k}}} \rightarrow 0$, as $k \rightarrow \infty$.

\subsubsection{Localized test functions}

While the space $\mathcal{S}$ is adequate for test fields produced by compact precipitates, it does not contain functions of the form (3.5) corresponding to nucleation of slabs. The proof of equivalence in Theorem 3.2, and especially Lemma 3.3 suggests that the test functions $\phi$ must satisfy

$$
\varliminf_{R \rightarrow \infty} \frac{\int_{A_{R}(h(R))}\left\{\frac{1}{h(R)^{2}}|\boldsymbol{\phi}-\boldsymbol{c}|^{2}+|\nabla \boldsymbol{\phi}|^{2}\right\} d \boldsymbol{x}}{\int_{B_{R}}|\nabla \boldsymbol{\phi}|^{2} d \boldsymbol{x}}=0
$$

for some constant $\boldsymbol{c} \in \mathbb{R}^{m}$, where $h(R)$ is a monotone increasing function, such that $h(R) / R \rightarrow 0$, as $R \rightarrow \infty$. Here

$$
A_{R}(h)=\left\{\boldsymbol{x} \in \mathbb{R}^{d}: R-h<|\boldsymbol{x}|<R\right\} .
$$

Without attempting to achieve the maximal extension, we can simplify the foregoing exposition by pointing out that in all of our applications we use only the functions $\phi(x)$ for which

$$
\lim _{R \rightarrow \infty} \frac{\int_{A_{R}(h(R))}|\nabla \phi|^{2} d \boldsymbol{x}}{\int_{B_{R}}|\nabla \phi|^{2} d \boldsymbol{x}}=0
$$


for every monotone increasing function $h(R)=o(R)$. This property can be restated as a relative uniform continuity of the function

$$
K(R)=\int_{B_{R}}|\nabla \phi|^{2} d \boldsymbol{x} .
$$

The notion of relative uniform continuity is the same as the classical notion of uniform continuity, except the absolute errors are replaced with relative errors. More precisely, $K(R)$ is relatively uniformly continuous if for every $\epsilon>0$ there exist $\delta>0$ such that for any $R_{1}>R_{2}>1$ for which $\left(R_{1}-R_{2}\right) / R_{1}<\delta$, we have $\left(K\left(R_{1}\right)-K\left(R_{2}\right)\right) / K\left(R_{1}\right)<\epsilon$. We can also restate this property using classical uniform continuity. Observe that the exponential function converts absolute errors into relative errors. Therefore, the relative uniform continuity of $K(R)$ is equivalent to the classical uniform continuity of $f(x)=\ln K\left(e^{x}\right)$ on $[0,+\infty)$.

Definition 3.5. We say that the test function $\phi \in W^{1, \infty}\left(\mathbb{R}^{d} ; \mathbb{R}^{m}\right)$ is localized if $K(R)$ is relatively uniformly continuous at infinity and there exists a constant $\boldsymbol{c} \in \mathbb{R}^{m}$, and a monotone increasing function $h(R)=o(R)$ such that

$$
\varliminf_{R \rightarrow \infty} \frac{1}{K(R) h(R)^{2}} \int_{A_{R}(h(R))}|\boldsymbol{\phi}-\boldsymbol{c}|^{2} d \boldsymbol{x}=0 .
$$

It is easy to see that any localized test function $\phi$ satisfies (3.16). One can also construct radial test functions $\phi(\boldsymbol{x})=u(|\boldsymbol{x}|)$ that satisfy (3.16) but are not localized in the sense of Definition 3.5.

Remark 3.6. If $d=1$ or 2, then the condition (3.18) is a consequence of (3.17) for any choice of c. If $d \geq 3$, then the condition (3.18) is not a consequence of (3.17).

Indeed, if $d=1$ we have

$$
\frac{1}{h(R)^{2}} \int_{A_{R}(h(R))}|\boldsymbol{\phi}-\boldsymbol{c}|^{2} d \boldsymbol{x} \leq \frac{\|\boldsymbol{\phi}-\boldsymbol{c}\|_{\infty}^{2}}{h(R)},
$$

and (3.18) follows as long as $h(R) \rightarrow \infty$, as $R \rightarrow \infty$. When $d=2$, we consider two cases. If

$$
\lim _{R \rightarrow \infty} K(R)<+\infty
$$

then $\phi \in \mathcal{S}$ and (3.18) follows from Lemma 3.7 below. If $K(R) \rightarrow+\infty$, as $R \rightarrow \infty$, then

$$
\frac{1}{h(R)^{2}} \int_{A_{R}(h(R))}|\boldsymbol{\phi}-\boldsymbol{c}|^{2} d \boldsymbol{x} \leq 3 \pi\|\boldsymbol{\phi}-\boldsymbol{c}\|_{\infty}^{2} \frac{R}{h(R)} .
$$

The relation (3.18) follows from Lemma B.1 proved in Appendix B, where

$$
\alpha(R)=\frac{1}{K(R)} \rightarrow 0, \text { as } R \rightarrow \infty .
$$

If $d \geq 3$, then the functions $\phi(\boldsymbol{x})=|\boldsymbol{x}|^{-\alpha}$ satisfy (3.17) but not (3.18), when $0<\alpha<(d-2) / 2$.

The terminology "localized test function" reflects the fact that these functions retain those features of the original smooth, compactly supported test functions that are essential for defining the binodal via the localization (2.6). The definition suggests that we may regard the test function $\boldsymbol{\phi}$ (or more precisely, $\boldsymbol{\phi}-\boldsymbol{c}$ ) as supported on a compact set $K \subset B_{R}$ for a sufficiently large $R$. This corresponds, via (2.6) to variations supported on a small ball $B_{\eta R}\left(\boldsymbol{x}_{0}\right) \subset \Omega$.

If we now wish to distinguish between the the binodal and the interior of the binodal region we need to further restrict our attention to the functions satisfying "zero volume fraction condition"

$$
\lim _{R \rightarrow \infty} f_{B_{R}}|\nabla \phi|^{2} d \boldsymbol{x}=0
$$

In the blow up problem (3.12), the condition (3.19) represents additional constraint on the behavior of the test function $\phi$ at infinity.

It is now natural to extend the space of admissible test functions from the space $\mathcal{S}$ to

$$
\mathcal{S}_{*}=\{\phi: \phi \text { is localized and satisfy (3.19) }\} .
$$


LEMMA 3.7. $\mathcal{S} \subset \mathcal{S}_{*}$.

The proof is in the Appendix B.

It is clear that for $\phi \in \mathcal{S}_{*}$ the integral in (3.12) does not have to converge. By analogy with (3.6) we replace it with the normalized functional

$$
I_{*}(\boldsymbol{F}, \boldsymbol{\phi})=\varlimsup_{R \rightarrow \infty} \frac{\int_{B_{R}} W^{\circ}(\boldsymbol{F}, \nabla \boldsymbol{\phi}) d \boldsymbol{x}}{\int_{B_{R}}|\nabla \boldsymbol{\phi}|^{2} d \boldsymbol{x}} .
$$

Observe that for any $\phi \in \mathcal{S}_{*}$ the functional $I_{*}(\boldsymbol{F}, \boldsymbol{\phi})$ is finite, since $\nabla \boldsymbol{\phi}(\boldsymbol{x})$ is uniformly bounded.

Theorem 3.8. The pair $\left(\mathcal{S}_{*}, I_{*}\right)$ characterizes the binodal.

Proof. If the inequality (2.12) fails for some $\phi \in C_{0}^{\infty}\left(B_{1} ; \mathbb{R}^{m}\right)$ then $I_{*}(\boldsymbol{F}, \boldsymbol{\phi})<0$, since $\phi \in \mathcal{S}_{*}$. Now assume that (2.12) is satisfied. Our goal is to prove that $I_{*}(\boldsymbol{F}, \boldsymbol{\phi}) \geq 0$ for all $\boldsymbol{\phi} \in \mathcal{S}_{*}$. Let us fix $\boldsymbol{\phi} \in \mathcal{S}_{*}$. Let $h(R)$ and $\boldsymbol{c} \in \mathbb{R}^{m}$ be as in the Definition 3.5. Let $\eta_{R}(\boldsymbol{x})$ be a Lipschitz cut-off function such that $0 \leq \eta_{R}(\boldsymbol{x}) \leq 1$, $\eta_{R}(\boldsymbol{x})=0$, when $|\boldsymbol{x}| \geq R$ and $\eta_{R}(\boldsymbol{x})=1$, when $|\boldsymbol{x}| \leq R-h(R)$. In addition we can choose $\eta_{R}(\boldsymbol{x})$ such that $\left|\nabla \eta_{R}(\boldsymbol{x})\right| \leq 1 / h(R)$. We have due to $(3.14)$

$$
\int_{B_{R}}\left|W^{\circ}\left(\boldsymbol{F}, \nabla\left(\eta_{R}(\boldsymbol{\phi}-\boldsymbol{c})\right)\right)-W^{\circ}(\boldsymbol{F}, \nabla \boldsymbol{\phi})\right| d \boldsymbol{x} \leq C \int_{A_{R}(h(R))}\left\{\frac{|\boldsymbol{\phi}-\boldsymbol{c}|^{2}}{h(R)^{2}}+|\nabla \boldsymbol{\phi}|^{2}\right\} d \boldsymbol{x} .
$$

Therefore,

$$
I_{*}(\boldsymbol{F}, \phi) \geq \varliminf_{R \rightarrow \infty} \frac{\int_{B_{R}} W^{\circ}\left(\boldsymbol{F}, \nabla\left(\eta_{R}(\phi-\boldsymbol{c})\right)\right) d \boldsymbol{x}}{\int_{B_{R}}|\nabla \boldsymbol{\phi}|^{2} d \boldsymbol{x}} \geq 0 .
$$

The theorem is proved.

The next logical step is to write down explicit conditions on $\phi \in \mathcal{S}_{*}$ minimizing (3.20). However, the definition of the functional $I_{*}(\boldsymbol{F}, \boldsymbol{\phi})$ makes it difficult to study its minima by classical variational methods. It is not even clear if the space $\mathcal{S}_{*}$ is a vector space. It is then natural to search for subsets of $\mathcal{S}_{*}$ that are vector spaces on which the functional $I_{*}(\boldsymbol{F}, \phi)$ can be represented by a classical variational integral functional without violating the pair-equivalence condition.

\subsubsection{Periodic-decaying test fields}

The analysis of spinodal in Section 3.1 suggests to consider the test fields that are periodic (or constant) in some directions and decaying in the remaining ones. More precisely, we choose our test functions $\boldsymbol{\phi}(\boldsymbol{x})$ to be in $\mathcal{S}$ "along" a $k$ dimensional subspace $\mathcal{L}$ of $\mathbb{R}^{d}$ and to have $(d-k)$ periods in the orthogonal complement $\mathcal{L}^{\perp}$.

More precisely, we assume that

$$
\phi\left(\boldsymbol{x}+\boldsymbol{u}_{j}\right)=\phi(\boldsymbol{x}), \quad\left\{\boldsymbol{u}_{1}, \ldots, \boldsymbol{u}_{d-k}\right\} \subset \mathcal{L}^{\perp} \text { is a basis of periods. }
$$

Let $\left\{\boldsymbol{e}_{1}, \ldots, \boldsymbol{e}_{k}\right\}$ be an orthonormal basis of $\mathcal{L}$ and $\left\{\boldsymbol{e}_{k+1}, \ldots, \boldsymbol{e}_{d}\right\}$ an orthonormal basis of $\mathcal{L}^{\perp}$. We define

$$
\boldsymbol{\psi}(\boldsymbol{t}, \boldsymbol{p})=\boldsymbol{\phi}\left(\sum_{j=1}^{k} t_{j} \boldsymbol{e}_{j}+\sum_{j=k+1}^{d} p_{j-k} \boldsymbol{e}_{j}\right), \quad \boldsymbol{t}=\left(t_{1}, \ldots, t_{k}\right) \in \mathbb{R}^{k}, \quad \boldsymbol{p}=\left(p_{1}, \ldots, p_{d-k}\right) \in Q_{d-k},
$$

where $Q_{d-k}$ is the period cell in $\boldsymbol{p}$ variables. We assume that $\boldsymbol{\psi} \in \mathcal{S}_{k}\left(Q_{d-k}\right)$, where

$$
\mathcal{S}_{k}\left(Q_{d-k}\right)=\left\{\boldsymbol{\psi} \in W^{1, \infty}\left(Y_{k} ; \mathbb{R}^{m}\right):\left[\boldsymbol{\psi}_{\boldsymbol{t}} \boldsymbol{\psi}_{\boldsymbol{p}}\right] \in L^{2}\left(Y_{k} ; \mathbb{R}^{m \times d}\right)\right\}, \quad Y_{k}=\mathbb{R}^{k} \times Q_{d-k} .
$$


Hence, we introduce the space of "periodic-decaying" test functions

$$
\mathcal{C}_{k}=\left\{\boldsymbol{\phi}(\boldsymbol{x})=\boldsymbol{\psi}(\boldsymbol{R} \boldsymbol{x}, \boldsymbol{Q} \boldsymbol{x}): \boldsymbol{\psi} \in \mathcal{S}_{k}\left(Q_{d-k}\right),\left[\begin{array}{l}
\boldsymbol{R} \\
\boldsymbol{Q}
\end{array}\right] \in S O(d), Q_{d-k}-\text { a parallelepiped }\right\} .
$$

The $k \times d$ matrix $\boldsymbol{R}$ has rows $\boldsymbol{e}_{1}, \ldots, \boldsymbol{e}_{k}$, while $(d-k) \times d$ matrix $\boldsymbol{Q}$ has rows $\boldsymbol{e}_{k+1}, \ldots, \boldsymbol{e}_{d}$. Observe that the sets $\mathcal{C}_{k}$ are the unions of the family of vector spaces smoothly parametrized by a finite dimensional manifold $\mathcal{G}_{d, k} \times G L(d-k, \mathbb{R}) / S L(d-k, \mathbb{Z})$. The first factor is the Grassmannian of $k$-dimensional subspaces $\mathcal{L} \subset \mathbb{R}^{d}$, while the second factor is the set of all distinct oriented Bravais lattices in $\mathbb{R}^{d-k}$. Here $G L(n, \mathbb{R})$ denotes the set of all invertible real $n \times n$ matrices, while $S L(n, \mathbb{Z})$ denotes the set of all $n \times n$ matrices with integer components and determinant equal to 1 . Such matrices map the lattice $\mathbb{Z}^{n}$ onto itself.

We remark that in the case $k=1$ the functions $\phi \in \mathcal{C}_{1}$ correspond to the physical idea of the nucleation of either a homogeneous plate (test field in $\overline{\mathcal{C}}_{1}$ ) $[81,80,24]$ or a composite plate $[97,98,99,27]$ while the case $k=d$ can be viewed as nucleation of a fully localized precipitate $[78,77,60,25,26]$.

We also distinguish special subspaces of $\mathcal{C}_{k}$ generated by functions $\boldsymbol{\psi} \in \mathcal{S}_{k}\left(Q_{d-k}\right)$ that do not depend on the $\boldsymbol{p}$ variables explicitly. We denote these subspaces by $\overline{\mathcal{S}}_{k}$ and $\overline{\mathcal{C}}_{k}$, respectively.

$$
\begin{gathered}
\overline{\mathcal{S}}_{k}=\left\{\boldsymbol{\psi} \in W^{1, \infty}\left(\mathbb{R}^{k} ; \mathbb{R}^{m}\right): \nabla \boldsymbol{\psi} \in L^{2}\left(\mathbb{R}^{k} ; \mathbb{R}^{m \times k}\right)\right\} ; \\
\overline{\mathcal{C}}_{k}=\left\{\boldsymbol{\phi}(\boldsymbol{x})=\boldsymbol{\psi}(\boldsymbol{R} \boldsymbol{x}): \boldsymbol{\psi} \in \overline{\mathcal{S}}_{k}, \boldsymbol{R}: \mathbb{R}^{d} \rightarrow \mathbb{R}^{k}, \boldsymbol{R} \boldsymbol{R}^{T}=\boldsymbol{I}_{k}\right\} .
\end{gathered}
$$

For example, the test functions in $\overline{\mathcal{C}}_{1}$ must have the form

$$
\phi(\boldsymbol{x})=\boldsymbol{f}((\boldsymbol{n}, \boldsymbol{x})), \quad \boldsymbol{f} \in W^{1, \infty}\left(\mathbb{R} ; \mathbb{R}^{m}\right), \quad \boldsymbol{f}^{\prime} \in L^{2}\left(\mathbb{R} ; \mathbb{R}^{m}\right),
$$

where $\boldsymbol{n} \in \mathbb{S}^{d-1}$ is constant but arbitrary. In physical terms these test functions correspond to the nucleation of long and thin platelets.

TheOREM 3.9. For any $1 \leq k \leq d$ we have $\mathcal{C}_{k} \subset \mathcal{S}_{*}$ and

$$
I_{*}(\boldsymbol{F}, \boldsymbol{\phi})=\frac{\int_{Y_{k}} W^{\circ}\left(\boldsymbol{F}, \boldsymbol{\psi}_{\boldsymbol{t}} \boldsymbol{R}+\boldsymbol{\psi}_{\boldsymbol{p}} \boldsymbol{Q}\right) d \boldsymbol{p} d \boldsymbol{t}}{\int_{Y_{k}}\left(\left|\boldsymbol{\psi}_{\boldsymbol{t}}\right|^{2}+\left|\boldsymbol{\psi}_{\boldsymbol{p}}\right|^{2}\right) d \boldsymbol{p} d \boldsymbol{t}}=\frac{\int_{Y} W^{\circ}(\boldsymbol{F}, \nabla \boldsymbol{\phi}) d \boldsymbol{x}}{\int_{Y}|\nabla \boldsymbol{\phi}|^{2} d \boldsymbol{x}},
$$

for any $\phi \in \mathcal{C}_{k}$, where $Y=\left[\boldsymbol{R}^{T} \boldsymbol{Q}^{T}\right] Y_{k}=\mathcal{L} \times \boldsymbol{Q}^{T} Q_{d-k}$.

The proof is in Appendix C.

Suppose now that $\boldsymbol{A} \in G L(d-k, \mathbb{R})$ maps $Q_{d-k}$ onto $[0,1]^{d-k}$. If $\boldsymbol{\psi} \in \mathcal{S}_{k}\left(Q_{d-k}\right)$, then

$$
\psi(t, p)=\psi_{0}(t, A p)
$$

where $\boldsymbol{\psi}_{0} \in \mathcal{S}_{k}\left([0,1]^{d-k}\right) \stackrel{\text { def }}{=} \mathcal{S}_{k}^{0}$. Since the denominator in (3.24) is always non-negative, the conclusion of our analysis is that the functional

$$
J_{k}(\boldsymbol{F}, \boldsymbol{\psi}, \boldsymbol{R}, \boldsymbol{Q}, \boldsymbol{A})=\int_{Y_{k}^{0}} W^{\circ}\left(\boldsymbol{F}, \boldsymbol{\psi}_{\boldsymbol{t}}(\boldsymbol{t}, \boldsymbol{p}) \boldsymbol{R}+\boldsymbol{\psi}_{\boldsymbol{p}}(\boldsymbol{t}, \boldsymbol{p}) \boldsymbol{A} \boldsymbol{Q}\right) d \boldsymbol{p} d \boldsymbol{t}
$$

defined on $\mathcal{S}_{k}^{0}$ is the desired replacement of the functional in (2.12). Here $Y_{k}^{0}=\mathbb{R}^{k} \times[0,1]^{d-k}$. Next we show that the spaces $\mathcal{C}_{k}$ contain enough test functions to characterize the binodal.

TheOREM 3.10. For any $1 \leq k \leq d$, any orthogonal splitting $\left[\boldsymbol{R}^{T} \boldsymbol{Q}^{T}\right] \in S O(d)$ of $\mathbb{R}^{d}$, and any $\boldsymbol{A} \in$ $G L(d-k, \mathbb{R})$ the pairs $\left(\mathcal{S}_{k}^{0}, J_{k}\right)$ characterize the binodal. 
Proof. Theorem 3.9 implies that if for given $\boldsymbol{R}, \boldsymbol{Q}$ and $\boldsymbol{A}$ there exists $\boldsymbol{\psi} \in \mathcal{S}_{k}^{0}$ for which $J_{k}(\boldsymbol{F}, \boldsymbol{\psi}, \boldsymbol{R}, \boldsymbol{Q})<0$, then the corresponding function $\phi(\boldsymbol{x})=\boldsymbol{\psi}(\boldsymbol{R} \boldsymbol{x}, \boldsymbol{A} \boldsymbol{Q} \boldsymbol{x}) \in \mathcal{S}_{*}$ satisfies $I_{*}(\boldsymbol{F}, \boldsymbol{\phi})<0$. By Theorem 3.8 we conclude that $\boldsymbol{F} \in \mathfrak{B}$.

Now assume that $\boldsymbol{F} \in \mathfrak{B}$. Then there exists $\phi \in C_{0}^{1}\left(B_{1} ; \mathbb{R}^{m}\right)$ for which the inequality (2.12) fails. Let us first extend the function $\phi(\boldsymbol{x})$ by zero to all of $\mathbb{R}^{d}$. Let us split the space $\mathbb{R}^{d}$ into an orthogonal sum $\mathbb{R}^{d}=$ $\boldsymbol{R}^{T} \mathbb{R}^{k} \oplus \boldsymbol{Q}^{T} \mathbb{R}^{d-k}$. Let $\boldsymbol{\psi}_{0}(\boldsymbol{t}, \boldsymbol{u})=\boldsymbol{\phi}\left(\boldsymbol{R}^{T} \boldsymbol{t}+\boldsymbol{Q}^{T} \boldsymbol{u}\right)$. Let $Q_{d-k}$ be the period cell mapped by $\boldsymbol{A} \in G L(d-k, \mathbb{R})$ onto $[0,1]^{d-k}$. Let $\boldsymbol{c} \in Q_{d-k}$ be the center of $Q_{d-k}$ and $a>0$ be so large that $\boldsymbol{\psi}_{0}(\boldsymbol{t}, \boldsymbol{u})=\mathbf{0}$, if $\boldsymbol{u} \notin a\left(Q_{d-k}-\boldsymbol{c}\right)$. Let $\widetilde{\boldsymbol{\psi}}(\boldsymbol{t}, \boldsymbol{u})$ be the $a\left(Q_{d-k}-\boldsymbol{c}\right)$-periodic extension of $\boldsymbol{\psi}_{0}(\boldsymbol{t}, \boldsymbol{u})$. Let $\boldsymbol{\psi}(\boldsymbol{t}, \boldsymbol{p})=a^{-1} \widetilde{\boldsymbol{\psi}}\left(a \boldsymbol{t}, a \boldsymbol{A}^{-1} \boldsymbol{p}\right)$. Then $\boldsymbol{\psi} \in \mathcal{S}_{k}^{0}$. Also

$$
J_{k}(\boldsymbol{F}, \boldsymbol{\psi}, \boldsymbol{R}, \boldsymbol{Q}, \boldsymbol{A})=a^{-d}|\operatorname{det} \boldsymbol{A}| \int_{B_{1}} W^{\circ}(\boldsymbol{F}, \nabla \boldsymbol{\phi}) d \boldsymbol{z}<0 .
$$

Remark 3.11. We observe that if $\boldsymbol{\phi} \in \mathcal{C}_{k}$ and $\lambda>0$, then $\phi_{\lambda}=\lambda \boldsymbol{\phi}(\boldsymbol{x} / \lambda) \in \mathcal{C}_{k}$, and

$$
\int_{Y} W^{\circ}\left(\boldsymbol{F}, \nabla \boldsymbol{\phi}_{\lambda}\right) d \boldsymbol{x}=\lambda^{d} \int_{Y} W^{\circ}(\boldsymbol{F}, \nabla \boldsymbol{\phi}) d \boldsymbol{x} .
$$

Therefore,

$$
\inf _{\boldsymbol{\phi} \in \mathcal{C}_{k}} J_{k}(\boldsymbol{F}, \boldsymbol{\phi})=-\operatorname{Ind}_{\mathfrak{B}^{c}}(\boldsymbol{F}),
$$

where $\operatorname{Ind}_{\mathfrak{B} c}(\boldsymbol{F})$ is the indicator function of the complement of the binodal region.

If $\phi \in \overline{\mathcal{C}}_{k}$ then the functional $J_{k}$ reduces to

$$
\bar{J}_{k}(\boldsymbol{F}, \boldsymbol{\psi}, \boldsymbol{R})=\int_{\mathbb{R}^{k}} W^{\circ}(\boldsymbol{F}, \nabla \boldsymbol{\psi}(\boldsymbol{t}) \boldsymbol{R}) d \boldsymbol{t} .
$$

Observe that the pairs $\left(\overline{\mathcal{C}}_{k}, \bar{J}_{k}\right)$ only bound the binodal, while the pairs $\left(\mathcal{S}_{k}^{0}, J_{k}\right)$ characterize it.

\subsubsection{Laminates}

Crossing the binodal may not be detectable by solving the Euler-Lagrange equations in one of the above problems. For instance, one can show that in the example considered in Section 5 with $d=2$ there are parts of the binodal that can only be detected by test functions whose gradient is supported on three specific gradients [49]. To construct such objects we need sequences of test functions in $\mathcal{C}_{1}$ that converge only weakly. The limiting value of the functional $J_{1}$ will then be expressed in terms of the finitely many parameters describing the geometry and piecewise-constant elastic fields in the limiting configuration.

More precisely, we consider elastic fields described by finitely supported probability measures $[28,62]$

$$
\nu=\sum_{j=1}^{r} \lambda_{j} \delta_{\boldsymbol{H}_{j}}, \quad \sum_{j=1}^{r} \lambda_{j}=1, \quad \lambda_{j}>0
$$

Given such a measure it is in general difficult to verify if $\nu$ is a gradient Young measure. However, one may easily construct a large class of such Young measures via the process of lamination [89].

Definition 3.12. Let $1 \leq j_{0} \leq r, s \in(0,1), \theta \in[0,1]$ and $\left\{\boldsymbol{B}_{1}, \boldsymbol{B}_{2}\right\} \subset \mathbb{M}$ are such that $\boldsymbol{B}_{1}-\boldsymbol{B}_{2}$ is rank-1 and $\boldsymbol{H}_{j_{0}}=s \boldsymbol{B}_{1}+(1-s) \boldsymbol{B}_{2}$. We say that the measure

$$
\nu^{\prime}=\nu+\theta \lambda_{j_{0}}\left(s \delta_{\boldsymbol{B}_{1}}+(1-s) \delta_{\boldsymbol{B}_{2}}-\delta_{\boldsymbol{H}_{j_{0}}}\right)
$$

is obtained from $\nu$ by lamination. 
Definition 3.13. A finite rank laminate is a finitely supported probability measure (3.27) for which there exists a sequence of probability measures $\nu_{1}, \ldots, \nu_{m}$, such that $\nu_{1}=\delta_{\boldsymbol{H}}, \nu_{m}=\nu$ and for each $k=1, \ldots, m-1$ the measure $\nu_{k+1}$ is obtained from $\nu_{k}$ by lamination.

For a measure $\nu$ given by (3.27) we define its "center of mass" by

$$
\bar{\nu}=\sum_{j=1}^{r} \lambda_{j} \boldsymbol{H}_{j} .
$$

We observe that if $\nu^{\prime}$ is obtained from $\nu$ by lamination then $\overline{\nu^{\prime}}=\bar{\nu}$. Hence, the matrix $\boldsymbol{H}$ in the definition of a finite rank laminate Young measure is equal to $\bar{\nu}$.

TheOREm 3.14. Suppose $\nu$ is a finite rank laminate with $\bar{\nu}=\boldsymbol{a} \otimes \boldsymbol{n}$. Then there exists a sequence $\left\{\boldsymbol{\phi}_{n}\right\} \subset \mathcal{C}_{1}$, such that $\boldsymbol{\phi}_{n} \rightarrow \phi_{0}$ uniformly, where $\boldsymbol{\phi}_{0}(\boldsymbol{x})$ is given by

$$
\phi_{0}(\boldsymbol{x})= \begin{cases}\boldsymbol{a}, & \text { if }(\boldsymbol{n}, \boldsymbol{x}) \geq 1, \\ \mathbf{0}, & \text { if }(\boldsymbol{n}, \boldsymbol{x}) \leq 0, \\ (\boldsymbol{n}, \boldsymbol{x}) \boldsymbol{a}, & \text { if } 0<(\boldsymbol{n}, \boldsymbol{x})<1,\end{cases}
$$

and such that

$$
\lim _{n \rightarrow \infty} J_{1}\left(\boldsymbol{F}, \phi_{n}, \boldsymbol{R}, \boldsymbol{Q}, \boldsymbol{I}\right)=J(\boldsymbol{F}, \nu)=\int_{\mathbb{M}} W^{\circ}(\boldsymbol{F}, \boldsymbol{H}) d \nu(\boldsymbol{H})=\sum_{j=1}^{r} \lambda_{j} W^{\circ}\left(\boldsymbol{F}, \boldsymbol{H}_{j}\right),
$$

where the $1 \times d$ matrix $\boldsymbol{R}$ can be identified with the unit vector $\boldsymbol{n}$.

The proof of Theorem 3.14 can be found in Appendix D.

We can now define the space

$$
\mathfrak{L}=\{\nu \text { - finite rank laminate Young measure, } \operatorname{rank}(\bar{\nu})=1\} .
$$

Corollary 3.15. The pair $(\mathfrak{L}, J(\boldsymbol{F}, \nu))$ bounds the binodal.

Proof. If $\boldsymbol{F} \notin \mathfrak{B}$ then $J_{1}\left(\boldsymbol{F}, \boldsymbol{\phi}_{n}, \boldsymbol{R}, \boldsymbol{Q}, \boldsymbol{I}\right) \geq 0$ for any $n \geq 1$, where the sequence $\left\{\boldsymbol{\phi}_{n}\right\}$ is as in Theorem 3.14. The formula (3.29) then implies that $J(\boldsymbol{F}, \nu) \geq 0$. Hence, the pair $(\mathfrak{L}, J(\boldsymbol{F}, \nu))$ bounds the binodal.

\section{Characterization}

In this section we use our equivalent formulations to derive explicit necessary conditions characterizing spinodal and bounding the binodal.

\subsection{Spinodal}

We recall that the deformation gradient $\boldsymbol{F}$ is weakly locally stable if and only if

$$
I_{\mathfrak{S}}(\boldsymbol{F}, \phi((\boldsymbol{x}, \boldsymbol{n})) \boldsymbol{a})=(\boldsymbol{A}(\boldsymbol{n} ; \boldsymbol{F}) \boldsymbol{a}, \boldsymbol{a}) \geq 0, \text { for all } \boldsymbol{a} \in \mathbb{R}^{m}, \boldsymbol{n} \in \mathbb{S}^{d-1} .
$$

The conditions of weak marginal stability can then be interpreted as the emergence of $\boldsymbol{a} \neq \mathbf{0}$ and $\boldsymbol{n} \in \mathbb{S}^{d-1}$ such that

$$
I_{\mathfrak{S}}(\boldsymbol{F}, \phi((\boldsymbol{x}, \boldsymbol{n})) \boldsymbol{a})=0 .
$$

If the above equality is satisfied because $\boldsymbol{F}$ crosses into the spinodal region, then the pair $(\boldsymbol{a}, \boldsymbol{n})$ must be minimizing for $I_{\mathfrak{S}}(\boldsymbol{F}, \phi((\boldsymbol{x}, \boldsymbol{n})) \boldsymbol{a})$. Therefore, the equilibrium equations

$$
\begin{gathered}
\nabla_{\boldsymbol{a}} I_{\mathfrak{S}}(\boldsymbol{F}, \phi((\boldsymbol{x}, \boldsymbol{n})) \boldsymbol{a})=\mathbf{0} \\
\nabla_{\boldsymbol{n}} I_{\mathfrak{S}}(\boldsymbol{F}, \phi((\boldsymbol{x}, \boldsymbol{n})) \boldsymbol{a})=\mathbf{0}
\end{gathered}
$$

must hold. 
Remark 4.1. The space $\mathcal{F}_{\mathfrak{S}}$ defined by (3.5) has $m+d-1$ degrees of freedom $\boldsymbol{a} \in \mathbb{R}^{m}$ and $\boldsymbol{n} \in \mathbb{S}^{d-1}$. The equation (4.2) describes equilibrium with respect to the variation of the local values of the field, as in the Euler-Lagrange equation. The equation (4.3) describes equilibrium with respect to "configurational" degrees of freedom $\boldsymbol{n}$ that describe the large-scale structure of the field (directions in which $\boldsymbol{\phi} \in \mathcal{F}_{\mathfrak{S}}$ does not decay at infinity). In this respect it is analogous to the Noether-Eshelby equation dealing with configuration of singularities, [92, 38, 39, 83, 45, 55]. Indeed, the lack of proper decay of the field $\boldsymbol{\phi}(\boldsymbol{x})$ can be regarded as a singularity at infinity.

One can see that equations (4.2)-(4.3) always have a family of trivial solutions $(\boldsymbol{a}, \boldsymbol{n})$, characterized by $\boldsymbol{a}=\mathbf{0}$. Hence, we may regard the problem of finding the non-trivial solutions of (4.2)-(4.3) as a bifurcation problem. Explicitly, equations (4.2)-(4.3) read

$$
\boldsymbol{A}(\boldsymbol{n} ; \boldsymbol{F}) \boldsymbol{a}=\mathbf{0}, \quad \boldsymbol{A}^{*}(\boldsymbol{a} ; \boldsymbol{F}) \boldsymbol{n}=\mathbf{0},
$$

where $\boldsymbol{A}^{*}(\boldsymbol{a} ; \boldsymbol{F})$ is the co-acoustic tensor defined as the linear map on $\mathbb{R}^{d}$ given by

$$
\boldsymbol{m} \mapsto \boldsymbol{A}^{*}(\boldsymbol{a} ; \boldsymbol{F}) \boldsymbol{m}=\left(W_{\boldsymbol{F} \boldsymbol{F}}(\boldsymbol{F})(\boldsymbol{a} \otimes \boldsymbol{m})\right)^{T} \boldsymbol{a} .
$$

Observe that the equations (4.4) are not independent. There is one relation between the left-hand sides in (4.4)

$$
\left(\boldsymbol{A}^{*}(\boldsymbol{a} ; \boldsymbol{F}) \boldsymbol{n}, \boldsymbol{n}\right)=(\boldsymbol{A}(\boldsymbol{n} ; \boldsymbol{F}) \boldsymbol{a}, \boldsymbol{a}) .
$$

Equations in (4.4) are also homogeneous in $\boldsymbol{a}$ and $\boldsymbol{n}$ and therefore, they can be regarded as $m+d-1$ constraints on $m d+(m-1)+(d-1)$ unknowns. As such they describe a co-dimension 1 surface in $\mathbb{M}$, which we can interpret as "an equation of spinodal".

Clearly, the points on the spinodal satisfy (4.4), however, the converse need not be true, i.e. some other points inside the spinodal region may satisfy (4.4) as well. It is possible to reduce the size of the system (4.4) by eliminating $\boldsymbol{a}$ in the case when $\operatorname{rank}(\boldsymbol{A}(\boldsymbol{n}))=m-1$. In that case the vector $\boldsymbol{a}$ spanning its kernel is determined up to a scalar multiple, or, if we normalize it to the unit length, up to a sign. Then

$$
\operatorname{cof}(\boldsymbol{A}(\boldsymbol{n}))=\alpha \boldsymbol{a} \otimes \boldsymbol{a}, \quad \alpha=\operatorname{Tr} \operatorname{cof}(\boldsymbol{A}(\boldsymbol{n})) \neq 0 .
$$

Using Einstein summation convention, the second equation in (4.4) and the first equation in (4.6) can be written as

$$
W_{F_{i \alpha} F_{j \beta}} a_{i} a_{j} n_{\beta}=0, \quad a_{i} a_{j}=\frac{1}{\alpha} \operatorname{cof}(\boldsymbol{A}(\boldsymbol{n}))_{i j},
$$

respectively. Hence, we obtain

$$
W_{F_{i \alpha} F_{j \beta}} \operatorname{cof}(\boldsymbol{A}(\boldsymbol{n}))_{i j} n_{\beta}=0 .
$$

We can write (4.7) in index-free notations

$$
(\operatorname{cof}(\boldsymbol{A}(\boldsymbol{n} ; \boldsymbol{F})), \mathbb{B}(\boldsymbol{n}, \boldsymbol{\eta} ; \boldsymbol{F}))=0, \quad \operatorname{Tr} \operatorname{cof}(\boldsymbol{A}(\boldsymbol{n} ; \boldsymbol{F})) \neq 0 .
$$

for all $\boldsymbol{\eta} \in \mathbb{R}^{d}$, if we introduce a bi-linear matrix-valued form

$$
\mathbb{B}(\boldsymbol{n}, \boldsymbol{\eta} ; \boldsymbol{F})_{i j}=W_{F_{i \alpha} F_{j \beta}}(\boldsymbol{F}) n_{\alpha} \eta_{\beta} .
$$

Conversely, if we take $\boldsymbol{\eta}=\boldsymbol{n}$ in (4.8) we obtain $\operatorname{det} \boldsymbol{A}(\boldsymbol{n})=0$. Hence, there exists $\boldsymbol{a} \neq \mathbf{0}$, such that the first equation in (4.4) is satisfied. The relation (4.6) also holds, since $\operatorname{Tr} \operatorname{cof}(\boldsymbol{A}(\boldsymbol{n})) \neq 0$. Thus, the second equation in (4.4) and the first equation in (4.8) are equivalent. We remark that the side condition $\operatorname{Tr} \operatorname{cof}(\boldsymbol{A}(\boldsymbol{n})) \neq 0$ in (4.8) is important, since for generic fully anisotropic tensors $W_{\boldsymbol{F} \boldsymbol{F}}$ the set $\{\boldsymbol{F} \in \mathbb{M}: \operatorname{rank}(\boldsymbol{A}(\boldsymbol{n} ; \boldsymbol{F})) \leq$ $m-2$ for some $|\boldsymbol{n}|=1\}$ has full dimension, if $d \geq 4$. We regard conditions (4.8) and (4.4) as generically equivalent, since at the spinodal we expect, in the generic case, the single smallest eigenvalue of $\boldsymbol{A}(\boldsymbol{n})$ to attain its minimum value of 0 .

It turns out that there are no other domain-local constraints on $\nabla \boldsymbol{y}(\boldsymbol{x})$ than $\nabla \boldsymbol{y}(\boldsymbol{x}) \notin \mathfrak{S}$ that follow from stability with respect to weak variations under the assumption of non-degeneracy of $\boldsymbol{A}(\boldsymbol{n} ; \boldsymbol{F})$. Indeed, due to [104] and the van Hove's theorem [116], the homogeneous deformation $\boldsymbol{y}_{0}(\boldsymbol{x})=\boldsymbol{F} \boldsymbol{x}$ is a weak local minimizer of (2.1) on the unit ball with Dirichlet boundary conditions, provided $\boldsymbol{A}(\boldsymbol{n} ; \boldsymbol{F})>0$ for all $|\boldsymbol{n}|=1$. 

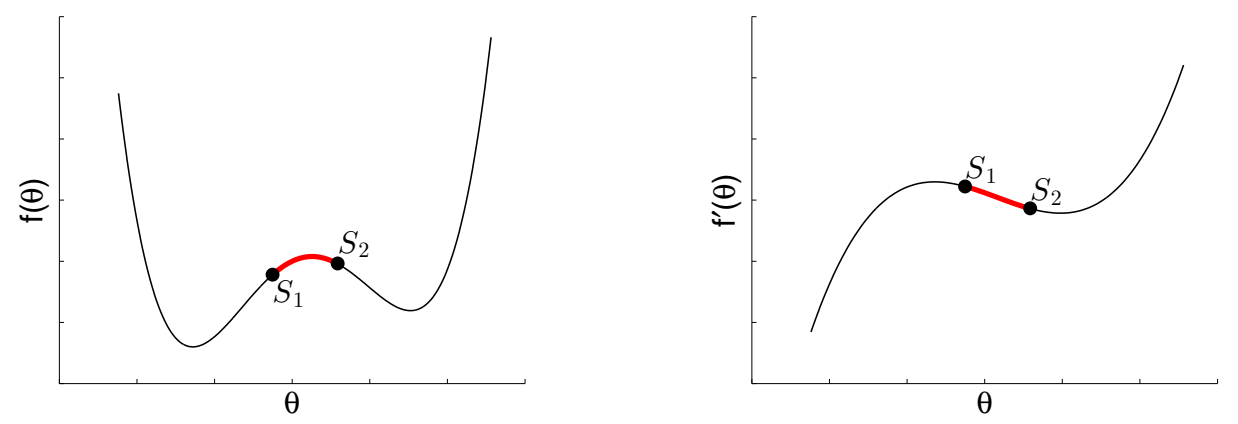

Figure 1: The double-well energy $f(\theta)$ from the example in Remark 4.2. Spinodal region is indicated by the bold line and spinodal points are $S_{1}$ and $S_{2}$.

Remark 4.2. As a simple illustration, consider the energy [21, 1]

$$
W(\boldsymbol{F})=f(\operatorname{Tr} \varepsilon)+\mu\left|\varepsilon-\frac{1}{d}(\operatorname{Tr} \boldsymbol{\varepsilon}) \boldsymbol{I}\right|^{2}, \quad \varepsilon=\frac{1}{2}\left(\boldsymbol{F}+\boldsymbol{F}^{T}\right), \quad \mu>0 .
$$

In this model the acoustic tensor can be written explicitly

$$
\boldsymbol{A}(\boldsymbol{n})=\mu\left(|\boldsymbol{n}|^{2} \boldsymbol{I}-\boldsymbol{n} \otimes \boldsymbol{n}\right)+\left(f^{\prime \prime}(\operatorname{Tr} \boldsymbol{\varepsilon})+2 \mu\left(1-\frac{1}{d}\right)\right) \boldsymbol{n} \otimes \boldsymbol{n} .
$$

From (4.5) we immediately find that $\boldsymbol{A}^{*}(\boldsymbol{a})=\boldsymbol{A}(\boldsymbol{a})$. The system (4.4) then becomes

$$
\left\{\begin{array}{l}
\mu|\boldsymbol{n}|^{2} \boldsymbol{a}+\left(f^{\prime \prime}(\operatorname{Tr} \boldsymbol{\varepsilon})+\mu\left(1-\frac{2}{d}\right)\right)(\boldsymbol{a}, \boldsymbol{n}) \boldsymbol{n}=\mathbf{0}, \\
\mu|\boldsymbol{a}|^{2} \boldsymbol{n}+\left(f^{\prime \prime}(\operatorname{Tr} \boldsymbol{\varepsilon})+\mu\left(1-\frac{2}{d}\right)\right)(\boldsymbol{a}, \boldsymbol{n}) \boldsymbol{a}=\mathbf{0} .
\end{array}\right.
$$

We see that $\boldsymbol{a}$ must be a scalar multiple of $\boldsymbol{n}$ and the system reduces to

$$
f^{\prime \prime}(\operatorname{Tr} \varepsilon)+2 \mu\left(1-\frac{1}{d}\right)=0 .
$$

The equation (4.8) can be written as

$$
d \mu^{d-1}\left(f^{\prime \prime}(\operatorname{Tr} \boldsymbol{\varepsilon})+2 \mu\left(1-\frac{1}{d}\right)\right) \boldsymbol{n}=\mathbf{0} .
$$

and it is clear that this equation is equivalent to (4.10). The actual spinodal in this example is the union of hyperplanes of the form $\operatorname{Tr} \varepsilon=\theta$, where $\theta$ is any zero of $\phi(\theta)=f^{\prime \prime}(\theta)+2 \mu(1-1 / d)$, around which $\phi(\theta)$ changes sign. The latter condition of transversality has to be imposed externally, since it is not captured by the equation (4.10). Our general theorems will feature such external transversality conditions, enabling us to assert the marginal stability of $\boldsymbol{F}$. If the function $f(\theta)$ has a double-well shape and its second derivative is shaped like a parabola, then the spinodal and the spinodal region are shown in Figure 1. We see how according to (4.10) the spinodal lies in the region, where $f^{\prime \prime}<0$.

\subsection{Binodal}

In this section we consider different explicit characterizations of the binodal. In particular we distinguish the PDE problem associated with nucleation of classical inclusions from the algebraic problem associated with nucleation of laminates. 


\subsubsection{Classical nucleation}

To obtain specific constraints on the value of $\boldsymbol{F}$ we need to study necessary conditions of equilibrium for the functionals $J_{k}, k=1, \ldots, d$, defined by (3.25). Before writing these conditions it is necessary to identify independent degrees of freedom associated with the spaces $\mathcal{C}_{k}: \psi \in \mathcal{S}_{k}^{0}$, the subspace $\mathcal{L} \subset \mathbb{R}^{d}$, described by the $k \times d$ matrix $\boldsymbol{R}$ satisfying $\boldsymbol{R R}^{T}=\boldsymbol{I}_{k}$, and the shape and orientation of the period cells $Q_{d-k}$ described by the matrix $\boldsymbol{A} \in G L(d-k, \mathbb{R})$. As in the case of the spinodal we identify the finite dimensional parameters $\boldsymbol{R}$ and $\boldsymbol{A}$ as configurational degrees of freedom associated with "singularities at infinity" The lack of rank-1 convexity of $W(\boldsymbol{F})$ allows the field variable $\boldsymbol{\psi} \in \mathcal{S}_{k}^{0}$ to possess additional configurational degrees of freedom associated with singularities allowed by the Euler-Lagrange equations at finite $\boldsymbol{x} \in \mathbb{R}^{d}$.

Our next two theorems introduce the classical Euler-Lagrange equations and the configurational NoetherEshelby equations $[92,38,39,83,45,55]$.

TheOREM 4.3. Assume that for $\boldsymbol{F} \in \mathfrak{B i n}$ there exists $1 \leq k \leq d$, orientation $\left[\boldsymbol{R}^{T} \boldsymbol{Q}^{T}\right] \in S O(d)$, a period cell shape $Q_{d-k}$ (i.e. $\boldsymbol{A} \in G L(d-k, \mathbb{R})$ ) and a non-zero function $\boldsymbol{\psi} \in \mathcal{S}_{k}^{0}$ such that $J_{k}(\boldsymbol{F}, \boldsymbol{\psi}, \boldsymbol{R}, \boldsymbol{Q}, \boldsymbol{A})=0$, while

$$
J_{k} \geq 0
$$

for all test functions $\phi \in \mathcal{C}_{k}$. Then

$$
\boldsymbol{F}+\nabla \boldsymbol{\phi}(\boldsymbol{x}) \notin \mathfrak{B} \text { for a.e. } \boldsymbol{x} \in \mathbb{R}^{d},
$$

and the test field $\boldsymbol{\phi}(\boldsymbol{x})=\boldsymbol{\psi}(\boldsymbol{R} \boldsymbol{x}, \boldsymbol{A Q \boldsymbol { x }})$ has to satisfy the Euler-Lagrange and the Noether-Eshelby equations

$$
\left\{\begin{array}{l}
\nabla \cdot \boldsymbol{P}(\boldsymbol{F}+\nabla \boldsymbol{\phi})=\mathbf{0}, \\
\nabla \cdot \boldsymbol{P}^{*}(\boldsymbol{F}+\nabla \boldsymbol{\phi})=\mathbf{0},
\end{array}\right.
$$

where ${ }^{4} \boldsymbol{P}(\boldsymbol{F})=W_{\boldsymbol{F}}(\boldsymbol{F})$, and $d^{5} \boldsymbol{P}^{*}(\boldsymbol{F})=W(\boldsymbol{F}) \boldsymbol{I}+\boldsymbol{F}^{T} \boldsymbol{P}(\boldsymbol{F})$. The optimal orientation and the period cell shape are determined by the additional conditions

$$
\int_{Y} \widehat{\boldsymbol{P}}^{*}(\nabla \boldsymbol{\phi}) d \boldsymbol{x}=\mathbf{0}
$$

where $\widehat{\boldsymbol{P}}^{*}(\boldsymbol{H})=W^{\circ}(\boldsymbol{F}, \boldsymbol{H}) \boldsymbol{I}-\boldsymbol{H}^{T} W_{\boldsymbol{H}}^{\circ}(\boldsymbol{F}, \boldsymbol{H})$.

Proof. By assumption, $\phi$ is the minimizer of the functional

$$
\phi \mapsto \int_{Y} \widehat{W}(\nabla \phi) d \boldsymbol{x}
$$

over all $\phi \in \mathcal{C}_{k}$, where

$$
\widehat{W}(\boldsymbol{H})=W^{\circ}(\boldsymbol{F}, \boldsymbol{H}) .
$$

The classical optimality conditions [10] then imply (4.11) and (4.12). Indeed,

$$
\widehat{\boldsymbol{P}}(\boldsymbol{H})=\widehat{W}_{\boldsymbol{H}}(\boldsymbol{H})=\boldsymbol{P}(\boldsymbol{F}+\boldsymbol{H})-\boldsymbol{P}(\boldsymbol{F}),
$$

which means that the Euler-Lagrange for the energy density (4.14) coincides with first equation in (4.12). We also compute

$$
\widehat{\boldsymbol{P}}^{*}(\boldsymbol{H})=\boldsymbol{P}^{*}(\boldsymbol{F}+\boldsymbol{H})+\boldsymbol{F}^{T} \boldsymbol{P}(\boldsymbol{F}+\boldsymbol{H})+\boldsymbol{N}(\boldsymbol{H}),
$$

\footnotetext{
3 The functions in the much larger space $\mathcal{S}_{*}$ would possess infinitely many configurational degrees of freedom at infinity corresponding to the infinite variety of possible asymptotic behaviors of $\phi \in \mathcal{S}_{*}$.

${ }^{4}$ In elasticity theory the tensor $\boldsymbol{P}(\boldsymbol{F})$ is called the Piola Kirchhoff stress tensor.

${ }^{5}$ In elasticity theory the tensor $\boldsymbol{P}^{*}(\boldsymbol{F})$ is called the Eshelby stress tensor.
} 
where

$$
\boldsymbol{N}(\boldsymbol{H})=\boldsymbol{H}^{T} \boldsymbol{P}(\boldsymbol{F})-(\boldsymbol{H}, \boldsymbol{P}(\boldsymbol{F})) \boldsymbol{I}-W(\boldsymbol{F}) \boldsymbol{I} .
$$

Therefore, the Noether-Eshelby equation $\nabla \cdot \widehat{\boldsymbol{P}}^{*}(\nabla \boldsymbol{\phi})=\mathbf{0}$ for the energy density (4.14) is equivalent to the second equation in (4.12), since $\nabla \cdot \boldsymbol{N}(\nabla \boldsymbol{\phi})=\mathbf{0}$ for any smooth vector field $\boldsymbol{\phi}$. Finally, (4.11) follows from a simple observation that $\boldsymbol{H}$ is a point of quasiconvexity for $\widehat{W}$ if and only if $\boldsymbol{F}+\boldsymbol{H}$ is a point of quasiconvexity for $W$.

The additional integral constraint (4.13) comes from variations in $\boldsymbol{R}, \boldsymbol{Q}$ and $\boldsymbol{A}$. If we fix $\boldsymbol{\psi}$ and $\boldsymbol{A}$, and vary $\left[\boldsymbol{R}^{T} \boldsymbol{Q}^{T}\right] \in S O(d)$ we obtain

$$
\int_{Y} \nabla \boldsymbol{\phi}^{T} \widehat{\boldsymbol{P}}(\nabla \boldsymbol{\phi}) d \boldsymbol{x} \in \operatorname{Sym}\left(\mathbb{R}^{d}\right) .
$$

Fixing $\boldsymbol{\psi}, \boldsymbol{R}$ and $\boldsymbol{Q}$ and varying $\boldsymbol{A}$ results in

$$
\int_{Y} \boldsymbol{Q} \nabla \boldsymbol{\phi}^{T} \widehat{\boldsymbol{P}}(\nabla \boldsymbol{\phi}) \boldsymbol{Q}^{T} d \boldsymbol{x}=\mathbf{0}
$$

By assumption

$$
\int_{Y} W^{\circ}(\boldsymbol{F}, \nabla \boldsymbol{\phi}) d \boldsymbol{x}=\frac{1}{|\operatorname{det} \boldsymbol{A}|} J_{k}(\boldsymbol{F}, \boldsymbol{\psi}, \boldsymbol{R}, \boldsymbol{Q}, \boldsymbol{A})=0 .
$$

This, together with (4.17) implies

$$
\int_{Y} \boldsymbol{Q} \widehat{\boldsymbol{P}}^{*}(\nabla \boldsymbol{\phi}) \boldsymbol{Q}^{T} d \boldsymbol{x}=\mathbf{0}
$$

Hence, in order to prove the theorem we need to show that

$$
\int_{Y} \boldsymbol{R} \widehat{\boldsymbol{P}}^{*}(\nabla \phi) \boldsymbol{Q}^{T} d \boldsymbol{x}=\mathbf{0}, \quad \int_{Y} \boldsymbol{R} \widehat{\boldsymbol{P}}^{*}(\nabla \boldsymbol{\phi}) \boldsymbol{R}^{T} d \boldsymbol{x}=\mathbf{0},
$$

since, according to (4.16) $\int_{Y} \widehat{\boldsymbol{P}}^{*} d \boldsymbol{x} \in \operatorname{Sym}\left(\mathbb{R}^{d}\right)$. The relation (4.13) then follows from Lemma 4.4 below.

Lemma 4.4. The equations (4.12) imply that

$$
\int_{Y} \widehat{\boldsymbol{P}}^{*}(\nabla \boldsymbol{\phi}) \boldsymbol{R}^{T} d \boldsymbol{x}=\mathbf{0}
$$

The proof is in the Appendix E.

Several remarks are in order. The first remark concerns the necessary condition (4.11).

Definition 4.5. If $\phi \in \mathcal{C}_{k} \backslash\{0\}$ satisfies (4.12) and (4.13), but fails (4.11) then $\phi$ is called a spurious solution.

The failure of quasiconvexity means that it is possible to modify the function $\phi$ locally, such that the modified function still belongs to $\mathcal{C}_{k}$, but gives a negative value to the functional $J_{k}$. This implies that $\boldsymbol{F} \in \mathfrak{B}$. In other words, spurious solutions do not correspond to points $\boldsymbol{F}$ on the binodal. Conversely, if $\phi$ satisfies (4.11)-(4.13), then obviously $\boldsymbol{F} \notin \mathfrak{B}$.

The Eshelby-Noether equation $(4.12)_{2}$ is the condition of equilibrium with respect to the degrees of freedom associated with the singularities of $\boldsymbol{\psi} \in \mathcal{S}_{k}^{0}$. Indeed, in the absence of singularities a well-know Noether identity $[92]$

$$
\nabla \cdot \boldsymbol{P}^{*}(\nabla \phi)=-(\nabla \phi)^{T} \nabla \cdot \boldsymbol{P}(\nabla \phi)
$$

says that $(4.12)_{2}$ is a consequence of $(4.12)_{1}$. If the singularities of $\boldsymbol{\psi} \in \mathcal{S}_{k}^{0}$ are smooth surfaces of jump discontinuity then the PDE (4.12) 2 can be replaced with an algebraic equation on the singular surface $\Sigma$ $[38,39,83,45,55,18]$

$$
\llbracket P^{*} \rrbracket n=0, \quad \boldsymbol{x} \in \Sigma,
$$


where $\llbracket \boldsymbol{P}^{*} \rrbracket=\boldsymbol{P}_{+}^{*}-\boldsymbol{P}_{-}^{*}$, is the jump of $\boldsymbol{P}^{*}(\boldsymbol{F}+\nabla \boldsymbol{\phi}(\boldsymbol{x}))$ across $\Sigma$. Here $\boldsymbol{n}$ is a unit normal to $\Sigma$. The region into which $\boldsymbol{n}$ points is called the "+" region, while the region from which $\boldsymbol{n}$ points is called the "-" region. It is well-known, the $d$ algebraic equations (4.20) can be reduced to a single scalar Maxwell relation [34, 38]

$$
p^{*}=\llbracket W \rrbracket-(\{\boldsymbol{P}\}, \llbracket \boldsymbol{F} \rrbracket)=0, \quad \boldsymbol{x} \in \Sigma,
$$

where $\{\boldsymbol{P}\}=\left(\boldsymbol{P}_{+}+\boldsymbol{P}_{-}\right) / 2$, interestingly (4.21) survives even in dynamics [114]. Now, if all the singularities of $\boldsymbol{\phi}(\boldsymbol{x})$ are smooth surfaces of jump discontinuity the system (4.12) is equivalent to the system $(4.12)_{1},(4.21)$. However, while the relation (4.21) will be convenient in the analysis of the example in Section 5 , in the general theory we are not making any assumptions on the nature of singularities of $\nabla \boldsymbol{\phi}(\boldsymbol{x})$, and the equation $(4.12)_{2}$ must be retained along with $(4.12)_{1}$.

It is clear that the verification of (4.11) may be difficult without the complete knowledge of the binodal. Yet, even partial knowledge of the binodal region can be used to demonstrate that condition (4.11) fails, thereby ruling out some of the spurious solutions of (4.12)-(4.13). For instance, there are several easyto-evaluate consequences of (4.11), especially on a smooth surface of jump discontinuity $\Sigma$ of $\nabla \phi$. These conditions are discussed in detail in [54]. One important example is the roughening equilibrium equation [51]

$$
\llbracket \boldsymbol{P}(\boldsymbol{F}+\nabla \boldsymbol{\phi}) \rrbracket^{T} \llbracket \nabla \boldsymbol{\phi} \rrbracket \boldsymbol{n}=\mathbf{0}, \quad \boldsymbol{x} \in \Sigma .
$$

This condition and the optimal orientation condition (4.13) are related via a localization argument. Indeed, consider the pair of fields $\boldsymbol{F}_{ \pm}=\boldsymbol{F}+\nabla \phi_{ \pm}\left(\boldsymbol{x}_{0}\right)$ at a point $\boldsymbol{x}_{0}$ on the surface of jump discontinuity. This implies a configuration, where an infinite slab carrying the field is $\boldsymbol{F}_{-}$is embedded in the infinite space where the field is $\boldsymbol{F}_{+}$. Such a configuration solves the $(4.12)_{1}$ if and only if

$$
\left\{\begin{array}{l}
\llbracket \boldsymbol{F} \rrbracket=\boldsymbol{a} \otimes \boldsymbol{n}, \text { for some } \boldsymbol{a} \in \mathbb{R}^{m} \\
\llbracket \boldsymbol{P} \rrbracket \boldsymbol{n}=\mathbf{0},
\end{array}\right.
$$

where $\boldsymbol{n}$ is the normal to the boundary of the slab. It solves $(4.12)_{2}$ if and only if (4.21) is satisfied and it satisfies (4.13) if and only if (4.22) holds.

Another consequence of (4.11) is the roughening stability inequality [51]. It is stated as

$$
\mathbb{C}_{ \pm}(\boldsymbol{a}, \boldsymbol{n})=\left[\begin{array}{cc}
\boldsymbol{A}_{ \pm}(\boldsymbol{n}) & \boldsymbol{B}_{ \pm}(\boldsymbol{a}, \boldsymbol{n})+\llbracket \boldsymbol{P} \rrbracket \\
\boldsymbol{B}_{ \pm}(\boldsymbol{a}, \boldsymbol{n})^{T}+\llbracket \boldsymbol{P} \rrbracket^{T} & \boldsymbol{A}_{ \pm}^{*}(\boldsymbol{a})
\end{array}\right] \geq 0
$$

in the sense of quadratic forms on the orthogonal complement of $\mathbb{R}[\boldsymbol{a},-\boldsymbol{n}]$. Here $\boldsymbol{A}_{ \pm}(\boldsymbol{n})=\boldsymbol{A}\left(\boldsymbol{n} ; \boldsymbol{F}_{ \pm}\right)$, $\boldsymbol{A}_{ \pm}^{*}(\boldsymbol{a})=\boldsymbol{A}^{*}\left(\boldsymbol{a} ; \boldsymbol{F}_{ \pm}\right)$and

$$
\boldsymbol{B}_{ \pm}(\boldsymbol{a}, \boldsymbol{n}) \boldsymbol{m}=\boldsymbol{A}\left(\boldsymbol{n}, \boldsymbol{m} ; \boldsymbol{F}_{ \pm}\right) \boldsymbol{a}=\left(W_{\boldsymbol{F} \boldsymbol{F}}\left(\boldsymbol{F}_{ \pm}\right)(\boldsymbol{a} \otimes \boldsymbol{m})\right) \boldsymbol{n}
$$

is the bilinear form satisfying $\boldsymbol{B}_{ \pm}(\boldsymbol{a}, \boldsymbol{n}) \boldsymbol{n}=\boldsymbol{A}_{ \pm}(\boldsymbol{n}) \boldsymbol{a}$ and $\boldsymbol{B}_{ \pm}^{T}(\boldsymbol{a}, \boldsymbol{n}) \boldsymbol{a}=\boldsymbol{A}_{ \pm}^{*}(\boldsymbol{a}) \boldsymbol{n}$.

The second remark concerns condition (4.13). Its compact general form comprises two relations: (4.18), which is a consequence of (4.12), and

$$
\int_{Y} \widehat{\boldsymbol{P}}^{*}(\nabla \boldsymbol{\phi}) \boldsymbol{Q}^{T} d \boldsymbol{x}=\mathbf{0}
$$

which is an algebraic condition of optimality with respect to orientation and period cell shape. We observe that there is an analogy between equations (4.12), (4.17) and the first equation in (4.4), and between (4.16) and the second equation in (4.4). Observe that if we dot the first equation in (4.4) with $\boldsymbol{a}$ we obtain that $I_{\mathfrak{S}}(\boldsymbol{F}, \phi((\boldsymbol{x}, \boldsymbol{n})) \boldsymbol{a})=0$ implying that generically $\boldsymbol{F}$ must lie in $\overline{\mathfrak{S}}$ - the closure of the spinodal region. However, it cannot describe the spinodal alone. Indeed, if $\boldsymbol{F}$ in the spinodal region is such that the function $\mathbb{S}^{d-1} \ni \boldsymbol{n} \mapsto \operatorname{det} \boldsymbol{A}(\boldsymbol{n} ; \boldsymbol{F})$ changes sign then there will be an entire neighborhood of $\boldsymbol{F}$ where this is true. Therefore, for each $\boldsymbol{F}$ with this property we can find $\boldsymbol{n} \neq \mathbf{0}$ with $\operatorname{det} \boldsymbol{A}(\boldsymbol{n} ; \boldsymbol{F})=0$. This shows that existence of non-trivial solutions of the first equation in (4.4) describes entire subregions of the spinodal region. The 
second equation in (4.4) eliminates most of these solutions and describes a co-dimension 1 surface containing the spinodal.

Our next theorem relates the existence of non-zero solutions to the system (4.12)-(4.13), i.e. the generalized bifurcation, with the marginal stability of $\boldsymbol{F}$. More precisely, we show that generically, the existence of nontrivial solutions implies that $\boldsymbol{F}$ must be either on the binodal or inside the binodal region. The solutions corresponding to the latter possibility are "spurious", and one must use both the partial knowledge of $\mathfrak{B}$ and computable consequences of (4.11), (see [54]), in order to eliminate them.

Theorem 4.6. Suppose $1 \leq k \leq d$ and $\boldsymbol{\phi} \in \mathcal{C}_{k}$ solves (4.12) and satisfies (4.13). Then $J_{k}(\boldsymbol{F}, \boldsymbol{\psi}, \boldsymbol{R}, \boldsymbol{Q}, \boldsymbol{A})=0$.

The proof is given in the Appendix F

Corollary 4.7. If (4.12) has solution $(\boldsymbol{\psi}, \boldsymbol{R}, \boldsymbol{Q}, \boldsymbol{A})$ such that $\boldsymbol{\psi} \neq \mathbf{0}$, and

$$
\frac{\partial J_{k}(\boldsymbol{F}, \boldsymbol{\psi}, \boldsymbol{R}, \boldsymbol{Q}, \boldsymbol{A})}{\partial \boldsymbol{F}} \neq \mathbf{0}
$$

then $\boldsymbol{F}$ must lie in the closure of $\mathfrak{B}$.

The Corollary 4.7 implies that $\boldsymbol{F}$ can not lie in the interior of the complement of $\mathfrak{B}$, so $\boldsymbol{F} \in \mathfrak{B i n}$. Therefore non-trivial solution of (4.12) corresponds to $\boldsymbol{F} \in \mathfrak{B i n}$ if and only if it is not spurious.

We give the following definition in order to distinguish parts of the binodal that could be identified by the test functions from $\mathcal{C}_{k}$.

Definition 4.8. We say that $\boldsymbol{F}$ belongs to the nucleation set $\mathfrak{N}_{k}$ if there exists a fundamental domain $Y=\mathcal{L} \times \boldsymbol{Q}^{T} Q_{d-k}$ such that the system (4.12) has a non-zero solution $\boldsymbol{\phi} \in \mathcal{C}_{k}$ satisfying (4.13).

In Section 5 we show that sometimes the sets $\mathfrak{N}_{k}$ can be characterized without a complete knowledge of the binodal set $\mathfrak{B}$.

Remark 4.9. As a simple illustration of a case where binodal can be fully characterized by our method, consider again the energy (4.9) The Euler-Lagrange equation from (4.12) becomes

$$
\mu \Delta \phi+\nabla\left(f^{\prime}(\operatorname{Tr} \boldsymbol{F}+\nabla \cdot \boldsymbol{\phi})+\frac{\mu(d-2)}{d} \nabla \cdot \boldsymbol{\phi}\right)=0 .
$$

Taking divergence of this equation we obtain

$$
\Delta \Phi^{\prime}(\operatorname{Tr} \boldsymbol{F}+\nabla \cdot \boldsymbol{\phi})=0, \quad \Phi(\theta)=f(\theta)+\frac{\mu(d-1)}{d} \theta^{2} .
$$

By assumption, $\nabla \cdot \boldsymbol{\phi}(\boldsymbol{x})$, and hence $\Phi^{\prime}(\operatorname{Tr} \boldsymbol{F}+\nabla \cdot \boldsymbol{\phi}(\boldsymbol{x}))$ is bounded on $\mathbb{R}^{d}$. Therefore, $\Phi^{\prime}(\operatorname{Tr} \boldsymbol{F}+\nabla \cdot \boldsymbol{\phi}(\boldsymbol{x}))=$ const. Taking the curl of (4.27) we obtain

$$
\Delta\left(\nabla \boldsymbol{\phi}-(\nabla \boldsymbol{\phi})^{T}\right)=\mathbf{0}
$$

in the sense of distributions. Hence, the boundedness of $\nabla \boldsymbol{\phi}(\boldsymbol{x})$ implies that $\nabla \boldsymbol{\phi}-(\nabla \boldsymbol{\phi})^{T}=2 \boldsymbol{M}$, where $\boldsymbol{M}$ is a constant anti-symmetric matrix. Therefore, $\boldsymbol{\phi}(\boldsymbol{x})=\boldsymbol{M} \boldsymbol{x}+\nabla h(\boldsymbol{x})$ for some locally integrable function $h(\boldsymbol{x})$. The boundedness of $\boldsymbol{\phi}(\boldsymbol{x})$ implies that $\boldsymbol{M}=\mathbf{0}$. Indeed, if $\boldsymbol{M} \neq \mathbf{0}$, there exists a unit vector $\boldsymbol{e}_{1}$ such that $\boldsymbol{M} \boldsymbol{e}_{1} \neq \mathbf{0}$. Let $\boldsymbol{e}_{2}=\boldsymbol{M} \boldsymbol{e}_{1} /\left|\boldsymbol{M} \boldsymbol{e}_{1}\right|$. Then, the unit vector $\boldsymbol{e}_{2}$ is orthogonal to $\boldsymbol{e}_{1}$, by anti-symmetry of $\boldsymbol{M}$, and $\left(\boldsymbol{M} \boldsymbol{e}_{1}, \boldsymbol{e}_{2}\right)=\left|\boldsymbol{M} \boldsymbol{e}_{1}\right|>0$. For any $R>0$ let $\boldsymbol{x}_{R}(t)=R \boldsymbol{e}_{1} \cos t+\operatorname{Re}_{2} \sin t$ be a closed loop. We conclude that

At the same time we have

$$
\left|\int_{0}^{2 \pi}\left(\boldsymbol{\phi}\left(\boldsymbol{x}_{R}(t)\right), \dot{\boldsymbol{x}}_{R}(t)\right) d t\right| \leq 2 \pi R\|\phi\|_{\infty}
$$

$$
\int_{0}^{2 \pi}\left(\nabla h\left(\boldsymbol{x}_{R}(t)\right), \dot{\boldsymbol{x}}_{R}(t)\right) d t=0, \quad \int_{0}^{2 \pi}\left(\boldsymbol{M} \boldsymbol{x}_{R}(t), \dot{\boldsymbol{x}}_{R}(t)\right) d t=2 \pi R^{2}\left(\boldsymbol{M} \boldsymbol{e}_{1}, \boldsymbol{e}_{2}\right)
$$



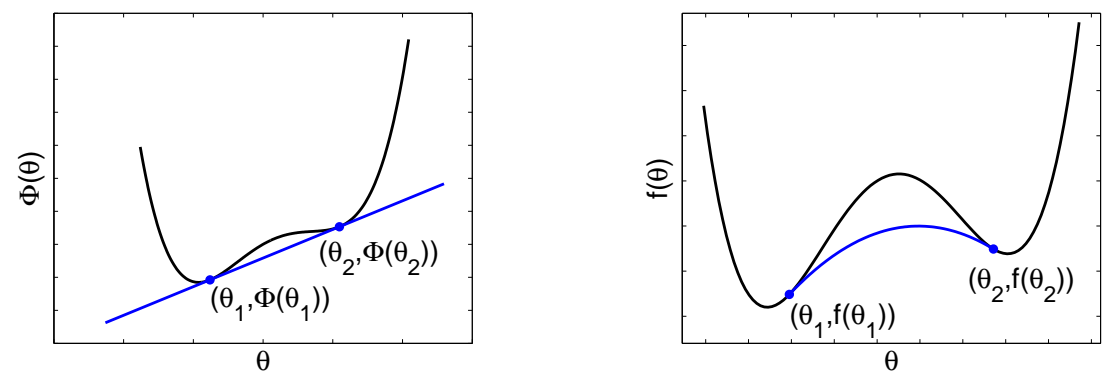

Figure 2: Common tangent to the graph of the function $\Phi(\theta)$ from Remark 4.9 and its image on the graph of the function $f(\theta)$.

Thus, $\phi(\boldsymbol{x})=\nabla h(\boldsymbol{x})$, while $\Phi^{\prime}(\operatorname{Tr} \boldsymbol{F}+\Delta h)=C=$ const. We claim that $C=\Phi^{\prime}(\operatorname{Tr} \boldsymbol{F})$. Indeed, if $C \neq \operatorname{Tr} \boldsymbol{F}$, then for every $\boldsymbol{x} \in \mathbb{R}^{d}$ the number $\nabla \cdot \boldsymbol{\phi}(\boldsymbol{x})=\Delta h(\boldsymbol{x})$ must belong to a finite set of solutions of the equation $\Phi^{\prime}(\operatorname{Tr} \boldsymbol{F}+\eta)=C$, none of which is zero. Hence, $|\nabla \boldsymbol{\phi}(\boldsymbol{x})|>\delta>0$ for all $\boldsymbol{x} \in \mathbb{R}^{d}$ for some positive number $\delta$. But then, condition (3.19) will not be satisfied. If the graph of function $f(\theta)$ has the shape shown in Figure 1 then the equation

$$
\Phi^{\prime}(\operatorname{Tr} \boldsymbol{F}+\eta)=\Phi^{\prime}(\operatorname{Tr} \boldsymbol{F})
$$

will either have a unique solution $\eta=0$, or three solutions, two of which $\eta_{1}$ and $\eta_{2}$ are non-zero. In the former case the Euler-Lagrange PDE (4.12) 1 has only trivial solutions in $\mathcal{S}_{*}$. In the latter case condition (4.11) helps us to rule out some of the spurious solutions. Observe that one of the three solutions of (4.29) is always inside the spinodal region. Hence, assuming that $\operatorname{Tr} \boldsymbol{F}$ is not in the spinodal region we only need to consider solutions of the form

$$
\Delta h=\eta \chi_{\Omega}(\boldsymbol{x}),
$$

where $\eta$ is the unique non-zero solution of (4.29) for which $\operatorname{Tr} \boldsymbol{F}+\eta$ is not in the spinodal region, and $\Omega$ is an arbitrary measurable subset of $\mathbb{R}^{d}$, satisfying "zero volume fraction condition"

$$
\lim _{R \rightarrow \infty} \frac{\left|\Omega \cap B_{R}\right|}{\left|B_{R}\right|}=0
$$

so that the corresponding solution $\phi$ satisfy (3.19). In this case any choice of an open and bounded subset $\Omega \subset \mathbb{R}^{d}$ provides a solution $\phi \in \mathcal{S}$ to (4.12) 1 via the solution $h \in H^{2}\left(\mathbb{R}^{d}\right)$ of (4.30).

The equation (4.12) $)_{2}$ is difficult to use directly in this example. Instead we restrict the class of solutions of the bifurcation system (4.12) only to those, where the set $\Omega$ has smooth boundary. In this case the equation (4.12) 2 can be replaced by the Maxwell relation (4.21)

$$
f(\operatorname{Tr} \boldsymbol{F}+\eta)-f(\operatorname{Tr} \boldsymbol{F})-f^{\prime}(\operatorname{Tr} \boldsymbol{F}) \eta+\mu\left|\llbracket \nabla \nabla h \rrbracket-\frac{1}{d} \llbracket \Delta h \rrbracket \boldsymbol{I}\right|^{2}=0 .
$$

Observe that due to the assumed smoothness of the boundary of $\partial \Omega$ and elliptic regularity we conclude that $\llbracket \nabla \nabla h \rrbracket$ must be a rank-1 matrix on $\partial \Omega$. Hence, $\llbracket \nabla \nabla h \rrbracket=\eta \boldsymbol{n}(\boldsymbol{x}) \otimes \boldsymbol{n}(\boldsymbol{x}), \boldsymbol{x} \in \partial \Omega$. Thus, we obtain

$$
f(\operatorname{Tr} \boldsymbol{F}+\eta)-f(\operatorname{Tr} \boldsymbol{F})-f^{\prime}(\operatorname{Tr} \boldsymbol{F}) \eta+\mu \eta^{2}\left(1-\frac{1}{d}\right)=0 .
$$

Rewriting this in terms of the function $\Phi(\theta)$ we obtain

$$
\Phi(\operatorname{Tr} \boldsymbol{F}+\eta)-\Phi(\operatorname{Tr} \boldsymbol{F})-\Phi^{\prime}(\operatorname{Tr} \boldsymbol{F}) \eta=0 .
$$


Equations (4.29) and (4.31) have a geometric interpretation. They say that the straight line connecting the points $(\operatorname{Tr} \boldsymbol{F}, \Phi(\operatorname{Tr} \boldsymbol{F}))$ and $(\operatorname{Tr} \boldsymbol{F}+\eta, \Phi(\operatorname{Tr} \boldsymbol{F}+\eta))$ on the graph of $\Phi(\theta)$ must be a common tangent at both points. Figure 2 shows that if $f(\theta)$ is as shown on Figure 1 then there is a unique common tangent to the graph of $\Phi(\theta)$, touching it at the points $\theta=\theta_{1}$ and $\theta=\theta_{2}$. Thus, either $\operatorname{Tr} \boldsymbol{F}=\theta_{1}$ and $\Delta h(\boldsymbol{x})=\left(\theta_{2}-\theta_{1}\right) \chi_{\Omega}(\boldsymbol{x})$, or $\operatorname{Tr} \boldsymbol{F}=\theta_{2}$ and $\Delta h(\boldsymbol{x})=\left(\theta_{1}-\theta_{2}\right) \chi_{\Omega}(\boldsymbol{x})$.

Suppose now that $\Omega$ is an arbitrary $(d-k)$-periodic array of arbitrary smooth inclusions where

$$
\Delta h(\boldsymbol{x})=\llbracket \theta \rrbracket \chi_{\Omega}(\boldsymbol{x}) .
$$

Computing Fourier transform in $\boldsymbol{t}$ variables and Fourier coefficients in $\boldsymbol{p}$ variables in (4.32) we can easily verify that $\phi \in \mathcal{C}_{k}$. It remains to verify condition (4.13). We have, after straightforward calculations, taking into account (4.29) and (4.31)

$$
\frac{1}{\mu} \widehat{\boldsymbol{P}}^{*}=\left(|\nabla \nabla h|^{2}-(\Delta h)^{2}\right) \boldsymbol{I}+2\left(\Delta h \nabla \nabla h-(\nabla \nabla h)^{2}\right) .
$$

Integration by parts gives

$$
\int_{Y}|\nabla \nabla h|^{2} d \boldsymbol{x}=\int_{Y}(\Delta h)^{2} d \boldsymbol{x}, \quad \int_{Y}(\nabla \nabla h)^{2} d \boldsymbol{x}=\int_{Y} \Delta h \nabla \nabla h d \boldsymbol{x} .
$$

Therefore, (4.13) is satisfied. Hence, the sets $\mathfrak{N}_{k}$ are all the same for all $k$ and are given by

$$
\mathfrak{N}_{k}=\left\{\boldsymbol{F}: \operatorname{Tr} \boldsymbol{F} \in\left\{\theta_{1}, \theta_{2}\right\}\right\},
$$

where $\theta_{1}, \theta_{2}$ are determined as $\theta$-coordinates of the two points of common tangency, as shown in Figure 2. In [53] we show that in this example $\mathfrak{B i n}=\mathfrak{N}_{k}$. It is also clear that if the graph of $\Phi(\theta)$ admits more than one common tangent, then the system (4.12)-(4.13) will also have spurious solutions corresponding to the interior points of the binodal region.

\subsubsection{Microstructure nucleation}

The main difference between the Legendre-Hadamard condition and the quasiconvexity condition is that in the former case the finite-dimensional set of test functions (3.5) "exhausts" possible localized instabilities. This leads to algebraic system (4.8), whose nontrivial solutions signal instability whenever $\boldsymbol{F}$ crosses the spinodal. Similarly, it may be intuitively appealing to think that crossing the binodal always manifests itself through the bifurcation in (4.12)-(4.13) in the class of decaying-periodic fields. However, we know that some points $\boldsymbol{F}$ on the binodal can be revealed only by studying nucleation of finite rank laminates. In this case the functional

$$
J(\boldsymbol{F}, \nu)=\sum_{i=1}^{r} \lambda_{j} W^{\circ}\left(\boldsymbol{F}, \boldsymbol{H}_{j}\right)
$$

is non-negative for any finite rank laminate $\nu$, with $\bar{\nu}=\boldsymbol{a} \otimes \boldsymbol{n}$, while achieving its minimum value of zero at a specific finite rank laminate, $\boldsymbol{a} \neq \mathbf{0}$ and $\boldsymbol{n} \in \mathbb{S}^{d-1}$. Finding the corresponding bounds for the binodal leads to an algebraic problem formulated below.

We recall that on each step of the construction of the measure $\nu$ in Definition 3.13 by means of lamination we introduced free parameters that can be varied in order to minimize $J(\boldsymbol{F}, \nu)$ given by (4.33). The equilibrium equations obtained from such a minimization are constraints on the matrices $\boldsymbol{H}_{j}$ and weights $\lambda_{j}$ in (3.27) get more and more complicated with the growth of the rank of the laminate. Below, we exhibit the recursive structure of the ensuing algebraic system by examining the passage from rank-1 to rank-two laminates.

The rank-1 laminate corresponds to $\nu_{1}=\delta_{\boldsymbol{a} \otimes \boldsymbol{n}}$. This Young measure is attained on the special test field $\phi_{0} \in \overline{\mathcal{C}}_{1}$ given by (3.28). In that case

$$
J\left(\boldsymbol{F}, \nu_{1}\right)=\bar{J}_{1}\left(\boldsymbol{F}, \phi_{0}, \boldsymbol{n}\right)=W^{\circ}(\boldsymbol{F}, \boldsymbol{a} \otimes \boldsymbol{n}) .
$$


The field value $\boldsymbol{F}$ is marginally stable if the following equations are satisfied

$$
\left\{\begin{array}{l}
W^{\circ}(\boldsymbol{F}, \boldsymbol{a} \otimes \boldsymbol{n})=0, \\
\nabla_{\boldsymbol{a}} W^{\circ}(\boldsymbol{F}, \boldsymbol{a} \otimes \boldsymbol{n})=\mathbf{0}, \\
\nabla_{\boldsymbol{n}} W^{\circ}(\boldsymbol{F}, \boldsymbol{a} \otimes \boldsymbol{n})=\mathbf{0} .
\end{array}\right.
$$

This system places $\boldsymbol{F}$ on the jump set $\mathfrak{J}$ (see [51]). The second rank laminate $\nu_{2}$ is obtained from $\nu_{1}$ by means of lamination in the sense of Definition 3.12.

$$
\nu_{2}=(1-\theta) \delta_{\boldsymbol{a} \otimes \boldsymbol{n}}+\theta s \delta_{\boldsymbol{H}_{1}}+\theta(1-s) \delta_{\boldsymbol{H}_{2}}, \quad s \boldsymbol{H}_{1}+(1-s) \boldsymbol{H}_{2}=\boldsymbol{a} \otimes \boldsymbol{n}, \quad \boldsymbol{H}_{2}-\boldsymbol{H}_{1}=\boldsymbol{b} \otimes \boldsymbol{m} .
$$

Observe that $J\left(\boldsymbol{F}, \nu_{2}\right)$ is affine in $\theta$. Hence, it is minimized either at $\theta=0$ corresponding to a rank- 1 laminate or at $\theta=1$. The goal of using rank- $r$ laminates is to capture points on the binodal that cannot be captured using rank- $(r-1)$ laminates. Therefore, we only need to consider the case $\theta=1$. Then

$$
J\left(\boldsymbol{F}, \nu_{2}\right)=s W^{\circ}(\boldsymbol{F}, \boldsymbol{a} \otimes \boldsymbol{n}-(1-s) \boldsymbol{b} \otimes \boldsymbol{m})+(1-s) W^{\circ}(\boldsymbol{F}, \boldsymbol{a} \otimes \boldsymbol{n}+s \boldsymbol{b} \otimes \boldsymbol{m}) .
$$

The field value $\boldsymbol{F}$ is marginally stable when the laminate with $s \in[0,1],\{\boldsymbol{a}, \boldsymbol{b}\} \subset \mathbb{R}^{m} \backslash\{\mathbf{0}\}$, and $\{\boldsymbol{n}, \boldsymbol{m}\} \subset \mathbb{S}^{d-1}$ delivers the global minimum to $J\left(\boldsymbol{F}, \nu_{2}\right)$, which is equal to 0 . Observe that both $s=0$ and $s=1$ correspond to rank-1 laminates, and are therefore excluded from the analysis of rank-2 laminates. Hence, we are interested only in the case when $s \in(0,1)$. If the minimum of $J\left(\boldsymbol{F}, \nu_{2}\right)$ is attained at a rank-2 laminate, then the following system of equations must hold

$$
\left\{\begin{array}{l}
s W\left(\boldsymbol{F}_{1}\right)+(1-s) W\left(\boldsymbol{F}_{2}\right)-W(\boldsymbol{F})-(\boldsymbol{P}(\boldsymbol{F}), \boldsymbol{a} \otimes \boldsymbol{n})=0 \\
W\left(\boldsymbol{F}_{2}\right)-W\left(\boldsymbol{F}_{1}\right)-\left(s \boldsymbol{P}\left(\boldsymbol{F}_{1}\right)+(1-s) \boldsymbol{P}\left(\boldsymbol{F}_{2}\right), \boldsymbol{F}_{2}-\boldsymbol{F}_{1}\right)=0 \\
\left(s \boldsymbol{P}\left(\boldsymbol{F}_{1}\right)+(1-s) \boldsymbol{P}\left(\boldsymbol{F}_{2}\right)-\boldsymbol{P}(\boldsymbol{F})\right) \boldsymbol{n}=\mathbf{0} \\
\left(s \boldsymbol{P}\left(\boldsymbol{F}_{1}\right)+(1-s) \boldsymbol{P}\left(\boldsymbol{F}_{2}\right)-\boldsymbol{P}(\boldsymbol{F})\right)^{T} \boldsymbol{a}=\mathbf{0} \\
\left(\boldsymbol{P}\left(\boldsymbol{F}_{2}\right)-\boldsymbol{P}\left(\boldsymbol{F}_{1}\right)\right) \boldsymbol{m}=\mathbf{0} \\
\left(\boldsymbol{P}\left(\boldsymbol{F}_{2}\right)-\boldsymbol{P}\left(\boldsymbol{F}_{1}\right)\right)^{T} \boldsymbol{b}=\mathbf{0}
\end{array}\right.
$$

where

$$
\boldsymbol{F}_{1}=\boldsymbol{F}+\boldsymbol{a} \otimes \boldsymbol{n}-(1-s) \boldsymbol{b} \otimes \boldsymbol{m}, \quad \boldsymbol{F}_{2}=\boldsymbol{F}+\boldsymbol{a} \otimes \boldsymbol{n}+s \boldsymbol{b} \otimes \boldsymbol{m}
$$

are the values of the deformation gradient in the internal laminate. There are $2 m+2 d$ independent equations in (4.35) with $2 m+2 d-1$ unknowns $s, \boldsymbol{a}, \boldsymbol{b}, \boldsymbol{m}$ and $\boldsymbol{n}$, where $\boldsymbol{n}$ and $\boldsymbol{m}$ are constrained to be unit vectors. We conclude that the system (4.35) restricts $\boldsymbol{F}$ to a co-dimension 1 surface corresponding to nucleation of second rank laminates.

Next we observe that

$$
\boldsymbol{F}_{2}-\boldsymbol{F}_{1}=\boldsymbol{b} \otimes \boldsymbol{n} .
$$

Therefore, in view of either the 5 th or the 6th equation in (4.35), we can rewrite the second equation in (4.35) as the Maxwell relation

$$
W\left(\boldsymbol{F}_{2}\right)-W\left(\boldsymbol{F}_{1}\right)-\left(\boldsymbol{P}\left(\boldsymbol{F}_{2}\right), \boldsymbol{F}_{2}-\boldsymbol{F}_{1}\right)=0,
$$

The system (4.35) can then be decomposed into two systems: the micro-level system and the macro-level system. The micro-level system consists of the 5th and the 6th equation in (4.35), as well as (4.36) and (4.37):

$$
\left\{\begin{array}{l}
W\left(\boldsymbol{F}_{2}\right)-W\left(\boldsymbol{F}_{1}\right)-\left(\boldsymbol{P}\left(\boldsymbol{F}_{2}\right), \boldsymbol{F}_{2}-\boldsymbol{F}_{1}\right)=0 \\
\left(\boldsymbol{P}\left(\boldsymbol{F}_{2}\right)-\boldsymbol{P}\left(\boldsymbol{F}_{1}\right)\right) \boldsymbol{m}=\mathbf{0} \\
\left(\boldsymbol{P}\left(\boldsymbol{F}_{2}\right)-\boldsymbol{P}\left(\boldsymbol{F}_{1}\right)\right)^{T} \boldsymbol{b}=\mathbf{0} \\
\boldsymbol{F}_{2}-\boldsymbol{F}_{1}=\boldsymbol{b} \otimes \boldsymbol{n} .
\end{array}\right.
$$


This is exactly the same system as in (4.34) defining the jump set $\mathfrak{J}$. In particular, $\left\{\boldsymbol{F}_{1}, \boldsymbol{F}_{2}\right\} \subset \mathfrak{J}$. The structure of the macro-level system becomes clear if we introduce the notations

$$
\overline{\boldsymbol{F}}=s \boldsymbol{F}_{1}+(1-s) \boldsymbol{F}_{2}, \quad \overline{\boldsymbol{P}}=s \boldsymbol{P}\left(\boldsymbol{F}_{1}\right)+(1-s) \boldsymbol{P}\left(\boldsymbol{F}_{2}\right), \quad \bar{W}=s W\left(\boldsymbol{F}_{1}\right)+(1-s) W\left(\boldsymbol{F}_{2}\right) .
$$

Then the macro-level system can be written as follows

$$
\left\{\begin{array}{l}
\bar{W}-W(\boldsymbol{F})-(\boldsymbol{P}(\boldsymbol{F}), \overline{\boldsymbol{F}}-\boldsymbol{F})=0 \\
(\overline{\boldsymbol{P}}-\boldsymbol{P}(\boldsymbol{F})) \boldsymbol{n}=\mathbf{0} \\
(\overline{\boldsymbol{P}}-\boldsymbol{P}(\boldsymbol{F}))^{T} \boldsymbol{a}=\mathbf{0} \\
\overline{\boldsymbol{F}}-\boldsymbol{F}=\boldsymbol{a} \otimes \boldsymbol{n} .
\end{array}\right.
$$

Observe that the system (4.40) has a structure very similar to structure of the system defining the jump set $\mathfrak{J}$, except the energy density $W(\boldsymbol{F})$ is replaced by a modified function $\bar{W}(\boldsymbol{F})$. To define this function we first introduce the set

$$
\widehat{\mathfrak{J}}=\left\{s \boldsymbol{F}_{1}+(1-s) \boldsymbol{F}_{2}: s \in[0,1], \boldsymbol{F}_{1}, \boldsymbol{F}_{2} \text { solve }(4.38)\right\} .
$$

Next, for $\overline{\boldsymbol{F}} \notin \widehat{\mathfrak{J}}$ we assume that $\bar{W}(\overline{\boldsymbol{F}})=W(\overline{\boldsymbol{F}})$, while for $\overline{\boldsymbol{F}} \in \widehat{\mathfrak{J}}$ we define

$$
\bar{W}(\overline{\boldsymbol{F}})=\min _{\overline{\boldsymbol{F}}=s \boldsymbol{F}_{1}+(1-s) \boldsymbol{F}_{2}}\left\{s W\left(\boldsymbol{F}_{1}\right)+(1-s) W\left(\boldsymbol{F}_{2}\right): \boldsymbol{F}_{1}, \boldsymbol{F}_{2} \text { solve }(4.38)\right\} .
$$

One can see that $\bar{W}(\boldsymbol{F})$ is a Lipschitz continuous function that agrees with $W(\boldsymbol{F})$ on the complement of $\widehat{\mathfrak{J}}$.

We claim now that $\boldsymbol{F}$ is located on the jump set of $\bar{W}(\boldsymbol{F})$, i.e.

$$
\bar{W}(\overline{\boldsymbol{F}})=\bar{W}, \quad \bar{W}_{\boldsymbol{F}}(\overline{\boldsymbol{F}})=\overline{\boldsymbol{P}},
$$

where $\overline{\boldsymbol{F}}, \overline{\boldsymbol{P}}$ and $\bar{W}$ are given by (4.39). We say that the point $\overline{\boldsymbol{F}} \in \widehat{\mathfrak{J}}$ is regular if the minimum in the definition of $\bar{W}(\overline{\boldsymbol{F}})$ is achieved at a unique pair $\boldsymbol{F}_{1}, \boldsymbol{F}_{2}$.

THEOREM 4.10. Assume that $\boldsymbol{F} \notin \widehat{\mathfrak{J}}$ and $\overline{\boldsymbol{F}} \in \widehat{\mathfrak{J}}$ is regular. Then, there are unique values $\boldsymbol{F}_{1}, \boldsymbol{F}_{2}$ and $s$ minimizing (4.41). Then, $\boldsymbol{F}_{1}$ and $\boldsymbol{F}_{2}$ are on the jump set $\mathfrak{J}$ of the energy $W(\boldsymbol{F})$, and $\boldsymbol{F}$ and $\overline{\boldsymbol{F}}$ are on the jump set $\overline{\mathfrak{J}}$ of the energy $\bar{W}(\boldsymbol{F})$ if and only if $\boldsymbol{F}, \boldsymbol{F}_{1}, \boldsymbol{F}_{2}$ and s solve (4.35).

Proof. By definition of $\boldsymbol{F}_{1}, \boldsymbol{F}_{2}$ the system (4.38) is satisfied. The system (4.40) places $\boldsymbol{F}$ and $\overline{\boldsymbol{F}}$ are on the jump set $\overline{\mathfrak{J}}$ of the energy $\bar{W}(\boldsymbol{F})$ if and only if (4.42) holds. To prove (4.42) we perturb a regular point $\overline{\boldsymbol{F}}$ within $\widehat{\mathfrak{J}}$. Then, the values $\boldsymbol{F}_{1}, \boldsymbol{F}_{2}$ and, hence, $\boldsymbol{b}$ and $\boldsymbol{m}$ will also be smoothly perturbed. Therefore,

$$
\delta \overline{\boldsymbol{F}}=\delta \boldsymbol{F}_{2}-s \delta \llbracket \boldsymbol{F} \rrbracket-(\delta s) \llbracket \boldsymbol{F} \rrbracket,
$$

where $\llbracket \boldsymbol{F} \rrbracket=\boldsymbol{F}_{2}-\boldsymbol{F}_{1}$. We also get

$$
\delta \bar{W}=\left(\boldsymbol{P}\left(\boldsymbol{F}_{2}\right), \delta \boldsymbol{F}_{2}\right)-(\delta s) \llbracket W \rrbracket-s\left(\left(\boldsymbol{P}\left(\boldsymbol{F}_{2}\right), \delta \boldsymbol{F}_{2}\right)-\left(\boldsymbol{P}\left(\boldsymbol{F}_{1}\right), \delta \boldsymbol{F}_{1}\right)\right) .
$$

Replacing $\llbracket W \rrbracket=W\left(\boldsymbol{F}_{2}\right)-W\left(\boldsymbol{F}_{1}\right)$ with $\left(\boldsymbol{P}\left(\boldsymbol{F}_{1}\right), \llbracket \boldsymbol{F} \rrbracket\right)$ and $\delta \boldsymbol{F}_{1}$ with $\delta \boldsymbol{F}_{2}-\delta \llbracket \boldsymbol{F} \rrbracket$ we obtain

$$
\delta \bar{W}=\left(\boldsymbol{P}\left(\boldsymbol{F}_{1}\right), \delta \overline{\boldsymbol{F}}\right)+(1-s)\left(\llbracket \boldsymbol{P} \rrbracket, \delta \boldsymbol{F}_{2}\right)=\left(\boldsymbol{P}\left(\boldsymbol{F}_{1}\right), \delta \overline{\boldsymbol{F}}\right)+(1-s)(\llbracket \boldsymbol{P} \rrbracket, \delta \overline{\boldsymbol{F}}),
$$

since

$$
(\llbracket \boldsymbol{P} \rrbracket, \llbracket \boldsymbol{F} \rrbracket)=(\llbracket \boldsymbol{P} \rrbracket, \delta \llbracket \boldsymbol{F} \rrbracket)=0,
$$

due to (4.38). Thus,

$$
\bar{W}_{\boldsymbol{F}}(\overline{\boldsymbol{F}})=s \boldsymbol{P}\left(\boldsymbol{F}_{1}\right)+(1-s) \boldsymbol{P}\left(\boldsymbol{F}_{2}\right)=\overline{\boldsymbol{P}} .
$$


We conclude that the set of field values $\boldsymbol{F}$ for which the system (4.35) has a non-trivial solutions can be interpreted as the jump set $\overline{\mathfrak{J}}$ for $\bar{W}(\boldsymbol{F})$ defined by (4.41). By replacing the function $W(\boldsymbol{F})$ with $\bar{W}(\boldsymbol{F})$, and by iterating this process, we can continue to define higher order jump sets for laminates of any rank. By analogy with (4.26) we also have a simple non-degeneracy condition.

THEOREM 4.11. If a finite rank laminate $\nu$ given (3.27) minimizes $J(\boldsymbol{F}, \nu)$ with the minimal value of zero then $\boldsymbol{F}$ must lie in the closure of $\mathfrak{B}$, provided

$$
\boldsymbol{A}(\boldsymbol{n} ; \boldsymbol{F}) \boldsymbol{a} \neq \mathbf{0}
$$

Proof. To prove the theorem it is enough to show that (4.43) guarantees that $\frac{\partial J(\boldsymbol{F}, \nu)}{\partial \boldsymbol{F}} \neq \mathbf{0}$. Indeed, we compute

$$
\frac{\partial J(\boldsymbol{F}, \nu)}{\partial \boldsymbol{F}}=\overline{\boldsymbol{P}}-\boldsymbol{P}(\boldsymbol{F})-W_{\boldsymbol{F} \boldsymbol{F}}(\boldsymbol{a} \otimes \boldsymbol{n}) \neq \mathbf{0}, \quad \overline{\boldsymbol{P}}=\int_{\mathbb{M}} \boldsymbol{P}(\boldsymbol{F}+\boldsymbol{H}) d \nu(\boldsymbol{H}) .
$$

It is clear that if

$$
\nu^{\prime}=\sum_{j=1}^{r} \lambda_{j} \delta_{\boldsymbol{H}_{j}^{\prime}}
$$

is a finite rank laminate with $\bar{\nu}^{\prime}=\mathbf{0}$, then the measure

$$
\nu=\sum_{j=1}^{r} \lambda_{j} \delta_{\boldsymbol{H}_{j}^{\prime}+\boldsymbol{a} \otimes \boldsymbol{n}}
$$

is also a finite rank laminate with $\bar{\nu}=\boldsymbol{a} \otimes \boldsymbol{n}$. Hence, if $\boldsymbol{H}_{j}^{\prime}$ are fixed, then the function

$$
j\left(\boldsymbol{a}, \boldsymbol{n}, \nu^{\prime}\right)=\sum_{j=1}^{r} \lambda_{j} W^{\circ}\left(\boldsymbol{F}, \boldsymbol{H}_{j}^{\prime}+\boldsymbol{a} \otimes \boldsymbol{n}\right)
$$

must be minimized in $\boldsymbol{a}$. Hence,

$$
\mathbf{0}=\nabla_{\boldsymbol{a}} j\left(\boldsymbol{a}, \boldsymbol{n}, \nu^{\prime}\right)=(\overline{\boldsymbol{P}}-\boldsymbol{P}(\boldsymbol{F})) \boldsymbol{n} .
$$

Therefore, according to (4.44),

$$
\frac{\partial J(\boldsymbol{F}, \nu)}{\partial \boldsymbol{F}} \boldsymbol{n}=\boldsymbol{A}(\boldsymbol{n} ; \boldsymbol{F}) \boldsymbol{a} .
$$

The theorem is now proved.

Observe that by minimizing $j\left(\boldsymbol{a}, \boldsymbol{n}, \nu^{\prime}\right)$ in $\boldsymbol{n}$ we also obtain

$$
(\overline{\boldsymbol{P}}-\boldsymbol{P}(\boldsymbol{F}))^{T} \boldsymbol{a}=\mathbf{0},
$$

which is the macro-level roughening equilibrium condition $(4.40)_{3}$. For an example where a part of the binodal can be captured only via the equations (4.35) we refer to [49].

The fact that the binodal of $\bar{W}(\boldsymbol{F})$ must lie in the closure of the binodal region of $W(\boldsymbol{F})$ is illustrated in Figures 3. The original energy $W(\boldsymbol{F})$ is shown by a solid line, the quasiconvexification - by a dashed line and $\bar{W}(\boldsymbol{F})$ - by a dotted line. The left figure illustrates the case, where the jump set (points $B_{1}$ and $B_{2}$ in the figure) captures the binodal, without $\bar{W}(\boldsymbol{F})$ necessarily capturing the values of $Q W(\boldsymbol{F})$. In the vicinity of points $B_{1}$ and $B_{2}$ the dashed and dotted lines may or may not coincide. The right figure shows a different situation, where the jump set is strictly inside the binodal region, while the binodal (points $B_{1}$ and $B_{2}$ in the figure) can only be delivered by studying other nucleation patterns, for instance, precipitates of a more general shape or higher rank laminates. 

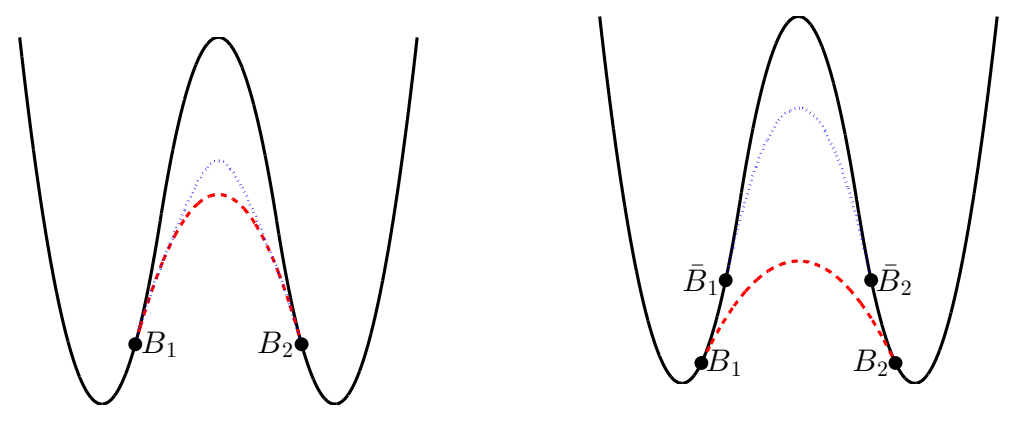

Figure 3: Possible relations between the envelopes $\bar{W}(\boldsymbol{F})$ and $Q W(\boldsymbol{F})$.

Remark 4.12. By the rank-one convexity of the quasiconvex envelope we have $Q W(\boldsymbol{F}) \leq \bar{W}(\boldsymbol{F})$ and $Q \bar{W}(\boldsymbol{F})=Q W(\boldsymbol{F})$. The points $\boldsymbol{F}$ corresponding to the non-trivial solutions of (4.12)-(4.13) with $W(\boldsymbol{F})$ replaced by $\bar{W}(\boldsymbol{F})$ can be regarded as unstable to the nucleation of composite precipitates represented by a continuously varying first rank laminate. The iteration process $W(\boldsymbol{F}) \rightarrow \bar{W}(\boldsymbol{F}) \rightarrow \overline{\bar{W}} \rightarrow \cdots$ brings additional flexibility to the binodal detection by allowing composite precipitates represented by a continuously parametrized rank-r laminates. Examples of such composite precipitates have been studied in [97, 98, 99, 126, 66, 65].

Remark 4.13. As a simple illustration of a complete characterization of the binodal by studying nucleation of simple laminates, we consider again our test case (4.9). Let us begin with the system of equations (4.34) describing the jump set. Straightforward calculations show that the third equation in (4.34) follows from the other two, and that the system can be reduced to the following one

$$
\operatorname{Tr} \boldsymbol{F}=\theta_{-}, \quad \boldsymbol{a}=\llbracket \theta \rrbracket \boldsymbol{n}, \quad \llbracket \Phi^{\prime}(\theta) \rrbracket=0, \quad \llbracket \Phi(\theta) \rrbracket-\llbracket \theta \rrbracket\left\{\Phi^{\prime}(\theta)\right\}=0,
$$

where $\Phi(\theta)$ is given in (4.28). We see that the jump set is characterized in terms of $\theta=\operatorname{Tr} \boldsymbol{F}$, where $\theta$ is a point where the tangent line to the graph of $\Phi(\theta)$ touches the graph at some other point, see Figure 2. For the double-well shape of $\Phi(\theta)$ shown in Figure 2. The jump set consists of the surfaces $\operatorname{Tr} \boldsymbol{F}=\theta_{1}$ and $\operatorname{Tr} \boldsymbol{F}=\theta_{2}$. This is the same set of points identified by the nucleation conditions obtained in Section 4.2.1. This is not surprising, since the analysis in Section 4.2.1 showed that the shape of the precipitate in this case can be arbitrary, including a slab used for computing the jump set. We also observe that each $\boldsymbol{F}=\boldsymbol{F}_{-} \in \mathfrak{J}$ can be paired with $\boldsymbol{F}_{+}=\boldsymbol{F}_{-}+\llbracket \theta \rrbracket \boldsymbol{n} \otimes \boldsymbol{n}$ for any unit vector $\boldsymbol{n}$.

Now a straightforward calculation gives the formula for $\bar{W}(\boldsymbol{F})$

$$
\bar{W}(\boldsymbol{F})= \begin{cases}W(\boldsymbol{F}), & \operatorname{Tr} \boldsymbol{F} \notin\left(\theta_{1}, \theta_{2}\right) \\ \bar{f}(\operatorname{Tr} \boldsymbol{\varepsilon})+\mu\left|\boldsymbol{\varepsilon}-\frac{1}{d}(\operatorname{Tr} \boldsymbol{\varepsilon}) \boldsymbol{I}\right|^{2}, & \operatorname{Tr} \boldsymbol{F} \in\left(\theta_{1}, \theta_{2}\right),\end{cases}
$$

where

$$
\bar{f}(\operatorname{Tr} \varepsilon)=\frac{\theta_{2}-\operatorname{Tr} \varepsilon}{\llbracket \theta \rrbracket} \Phi\left(\theta_{1}\right)+\frac{\operatorname{Tr} \varepsilon-\theta_{1}}{\llbracket \theta \rrbracket} \Phi\left(\theta_{2}\right)-\frac{\mu(d-1)}{d}(\operatorname{Tr} \varepsilon)^{2} .
$$

One can show that in fact $\bar{W}(\boldsymbol{F})=Q W(\boldsymbol{F})$ [53], which means that, in this case, studying simple laminates is sufficient for a complete characterization of the binodal.

\section{Bi-quadratic energy}

In this section we apply our approach to a nontrivial example where a rather complete picture of the binodal can be obtained by studying several specific families of test functions. The spinodal in this example is degenerate and will not be the focus of our analysis. 
More specifically we consider the bi-quadratic energy density of the form

$$
W(\boldsymbol{F})=\min \left\{f_{+}(\varepsilon), f_{-}(\varepsilon)\right\}, \quad \varepsilon=\frac{1}{2}\left(\boldsymbol{F}+\boldsymbol{F}^{T}\right), \quad f_{ \pm}(\varepsilon)=\frac{1}{2}\left(\mathrm{C}_{ \pm} \varepsilon, \varepsilon\right)+w_{ \pm},
$$

We assume that the elastic tensors $C_{ \pm}$are isotropic:

$$
\mathrm{C}_{ \pm} \boldsymbol{\xi}=\lambda_{ \pm}(\operatorname{Tr} \boldsymbol{\xi}) \boldsymbol{I}+2 \mu_{ \pm} \boldsymbol{\xi}, \quad \text { for any } \boldsymbol{\xi} \in \operatorname{Sym}\left(\mathbb{R}^{d}\right),
$$

and elliptic

$$
\lambda_{+}+2 \mu_{+}>0, \quad \lambda_{-}+2 \mu_{-}>0 .
$$

Additionally we assume that

$$
\lambda_{ \pm}+\mu_{ \pm} \neq 0, \quad \llbracket \mu \rrbracket \neq 0, \quad k \llbracket \lambda \rrbracket+2 \llbracket \mu \rrbracket \neq 0 .
$$

This energy plays an important role both in the mathematical theory of composite materials $[79,46,25,26,22]$ and in the modeling of martensitic phase transitions [70, 41, 42].

Even in this piece wise linear example we can not find all solutions to the system (4.12)-(4.13). However, we can obtain bounds on the binodal by computing in Section 5.1 nucleating solutions in $\overline{\mathcal{C}}_{k}, 1 \leq k \leq d$ that have ellipsoidal $k$-dimensional cross-section. In Section 5.2 we also present an example of a solution in $\mathcal{C}_{1} \backslash \overline{\mathcal{C}}_{1}$ in $2 \mathrm{D}$.

\section{$5.1 \quad$ Isolated cylindrical inclusions}

The goal of this section is to obtain bounds on the binodal in arbitrary dimensions using elliptical cylinders as test functions. Expanding on prior work $[78,77,36,17,60,70]$ we can compute the solutions of (4.12) for the bi-quadratic energy (5.1) corresponding to infinite elliptical cylinders explicitly. The explicit representation of these test functions in $[72,71,73]$ allows us to estimate their decay at infinity and prove that they are in $\overline{\mathcal{C}}_{k}$, so that our general theory applies. In $2 \mathrm{D}$ these solutions can be viewed as limiting cases of composite strips computed in Section 5.2, as the period $p$ goes to infinity.

For each $k \geq 1$ we will look for a solution of the system (4.12) in the form of an elliptical $k$-cylinder. We therefore define the sets $\mathfrak{N}_{k}^{\text {ell }}$ as in Definition 4.8.

Definition 5.1. We say that $\varepsilon$ belongs to the elliptical $k$-cylinder nucleation set $\mathfrak{N}_{k}^{\text {ell }}, k=1, \ldots, d$, if there exists an elliptical $k$-cylinder inclusion satisfying (4.12) and (4.13).

Observe that for $k=1$ such a cylinder is a plate, while for $k=d$, it is an ellipsoid. Moreover, general elliptical $k$-cylinders can be regarded as ellipsoids with some of the aspect ratios going to infinity [70, 40] This suggests that in order to map the entire binodal it is enough to consider only ellipsoids. However, the parts of the binodal identified by the elliptical cylinders are of the same dimension $(m d-1)$ as the parts of the binodal identified by the ellipsoids. Thus, for the strategy of passing to the limits in the space of ellipsoid parameters to succeed one needs to test against arbitrary ellipsoids and then optimize the explicit results over orientations and aspect ratios, allowing infinite values of the parameters to capture plates and cylinders. It is a challenging technical problem. Our approach, that treats elliptical $k$ and $r$-cylinders as distinct, when $k \neq r$, has the advantage of identifying singular optimal shapes directly.

If we align our coordinate system with the cylindrical inclusion in such a way that vectors $\boldsymbol{e}_{1}, \ldots, \boldsymbol{e}_{d}$ are directed along the coordinate axes, then we can write $\boldsymbol{x}=\left(t_{1}, \ldots, t_{k}, x_{k+1}, \ldots, x_{d}\right)$. The test field $\boldsymbol{\phi}(\boldsymbol{x})=\boldsymbol{\psi}(\boldsymbol{t})$ and we write the field $\varepsilon$ at infinity in the block form

$$
\varepsilon=\left[\begin{array}{cc}
\varepsilon_{0} & \boldsymbol{E}^{T} \\
\boldsymbol{E} & \boldsymbol{\varepsilon}^{\prime}
\end{array}\right],
$$

where $\varepsilon_{0}$ is a $k \times k$ matrix, $\boldsymbol{E}$ is a $(d-k) \times k$ matrix and $\varepsilon^{\prime}$ is a $(d-k) \times(d-k)$ matrix. We also use the notation

$$
\lambda(\boldsymbol{t})=\lambda_{-}(1-\chi(\boldsymbol{t}))+\lambda_{+} \chi(\boldsymbol{t}), \quad \mu(\boldsymbol{t})=\mu_{-}(1-\chi(\boldsymbol{t}))+\mu_{+} \chi(\boldsymbol{t}),
$$


where $\chi(\boldsymbol{t})$ is the characteristic function of the elliptical cylinder. One can see that the label "+" refers to the materials and fields inside the inclusion, while the label "-" refers to the materials and fields outside the inclusion. Finally, let $\mathrm{C}(\boldsymbol{t})$ be the local elasticity tensor defined by its action on an arbitrary strain $\boldsymbol{\xi}$ by

$$
\mathrm{C}(\boldsymbol{t}) \boldsymbol{\xi}=\lambda(\boldsymbol{t})(\operatorname{Tr} \boldsymbol{\xi}) \boldsymbol{I}+2 \mu(\boldsymbol{t}) \boldsymbol{\xi}, \quad \text { for any } \boldsymbol{\xi} \in \operatorname{Sym}\left(\mathbb{R}^{d}\right) .
$$

We observe that the elastic tensor $\mathrm{C}(\boldsymbol{t})$ and the elastic constants $\lambda(\boldsymbol{t})$ and $\mu(\boldsymbol{t})$ are piecewise constant with a jump discontinuity across the boundary of the elliptical $k$-cylinder.

\subsubsection{Euler-Lagrange equations}

The isotropy of the materials cause Euler-Lagrange equation in (4.12) to decouple into separate PDEs for $\boldsymbol{\psi}_{0}(\boldsymbol{t})=\left(\psi_{1}(\boldsymbol{t}), \ldots, \psi_{k}(\boldsymbol{t})\right)$ and $\boldsymbol{\psi}^{\prime}(\boldsymbol{t})=\left(\boldsymbol{\psi}_{k+1}(\boldsymbol{t}), \ldots, \psi_{d}(\boldsymbol{t})\right)$ :

$$
\left\{\begin{array}{l}
\nabla \cdot \mathrm{C}(\boldsymbol{t})\left(e\left(\boldsymbol{\psi}_{0}\right)+\varepsilon_{0}+\frac{\llbracket \lambda \rrbracket \operatorname{Tr} \boldsymbol{\varepsilon}^{\prime}}{k \llbracket \lambda \rrbracket+2 \llbracket \mu \rrbracket} \boldsymbol{I}_{k}\right)=\mathbf{0}, \\
\nabla \cdot \mu(\boldsymbol{t})\left(\nabla \boldsymbol{\psi}^{\prime}+2 \boldsymbol{E}\right)=0 .
\end{array}\right.
$$

The equations (5.3) decouple into equations of elasticity in $\mathbb{R}^{k}$ and an additional generalized anti-plane shear. The elastic strain field

$$
e(\boldsymbol{\phi})=\left[\begin{array}{cc}
e\left(\boldsymbol{\psi}_{0}\right) & \frac{1}{2}\left(\nabla \boldsymbol{\psi}^{\prime}\right)^{T} \\
\frac{1}{2} \nabla \boldsymbol{\psi}^{\prime} & \mathbf{0}
\end{array}\right]
$$

is smooth inside and outside of the elliptical $k$-cylinder

$$
\sum_{i=1}^{k} \frac{t_{k}^{2}}{a_{k}^{2}}=1
$$

but has a jump discontinuity across its boundary. Thus, in the application of the general theory we may replace the Noether-Eshelby equation $(4.12)_{2}$ with the Maxwell relation (4.21) on the boundary of the cylinder.

We know that both $e\left(\boldsymbol{\psi}_{0}\right)$ and $\nabla \boldsymbol{\psi}^{\prime}(\boldsymbol{t})$ are uniform inside the ellipsoid [90]. The values of these fields are determined uniquely by the fields at infinity and the shape of the ellipsoid described by the $k \times k$ matrix $\boldsymbol{a}=\operatorname{diag}\left(a_{1}, \ldots, a_{k}\right)$. Eshelby [36, 37] has presented the solution for 3D isotropic ellipsoidal inclusions in the isotropic external medium. We will use the elegant formulas that are valid in any dimensions and for general anisotropic media due to Kunin and Sosnina [73].

Recall the definition of the fourth order tensor $\mathrm{K}_{\mathrm{C}}(\boldsymbol{n})$, which is a Fourier space representation of the fundamental solution for the equations of linear elasticity in the general anisotropic medium C. Suppose $\boldsymbol{u}(\boldsymbol{t})$ solves

$$
\nabla \cdot \operatorname{Ce}(\boldsymbol{u})=\nabla \cdot \boldsymbol{\tau}, \quad \boldsymbol{t} \in \mathbb{R}^{k}
$$

where the symmetric external stress field $\boldsymbol{\tau}(\boldsymbol{t})$ is smooth and compactly supported. Then the Fourier transform of the strain will satisfy

$$
\widehat{e(\boldsymbol{u})}(\boldsymbol{\omega})=\mathrm{K}_{\mathrm{C}}(\boldsymbol{\omega}) \widehat{\boldsymbol{\tau}}(\boldsymbol{\omega})
$$

Explicitly,

$$
\mathrm{K}_{\mathrm{C}}(\boldsymbol{n}) \boldsymbol{\xi}=\boldsymbol{A}_{\mathrm{C}}(\boldsymbol{n})^{-1} \boldsymbol{\xi} \boldsymbol{n} \odot \boldsymbol{n},
$$

where $\boldsymbol{A}_{\mathrm{C}}(\boldsymbol{n})$ is the acoustic tensor of $\mathrm{C}$.

Theorem 5.2 (Kunin and Sosnina). 
(a) Suppose that $\boldsymbol{u}: \mathbb{R}^{k} \rightarrow \mathbb{R}^{k}$ satisfies

$$
\nabla \cdot \mathrm{C}(\boldsymbol{t})\left(e(\boldsymbol{u})+\boldsymbol{\varepsilon}^{\infty}\right)=\mathbf{0}
$$

where $\mathrm{C}(\boldsymbol{t})=(1-\chi(\boldsymbol{t})) \mathrm{C}_{-}+\chi(\boldsymbol{t}) \mathrm{C}_{+}$and $\chi(\boldsymbol{t})$ is the characteristic function of the ellipsoid (5.4). Then

$$
\varepsilon^{\infty}=\varepsilon^{+}+\left\langle\mathrm{K}_{\mathrm{C}_{-}}(\boldsymbol{n})\right\rangle_{a} \llbracket \mathrm{C} \rrbracket \varepsilon^{+}
$$

where $\varepsilon^{+}=e\left(\boldsymbol{u}_{+}\right)+\varepsilon^{\infty}$ is the strain field in the inclusion, and

$$
\left\langle\mathrm{K}_{\mathrm{C}_{-}}(\boldsymbol{n})\right\rangle_{\boldsymbol{a}}=f_{\mathbb{S}^{k-1}} \mathrm{~K}_{\mathrm{C}_{-}}\left(\boldsymbol{a}^{-1} \boldsymbol{n}\right) d S(\boldsymbol{n}), \quad \boldsymbol{a}=\operatorname{diag}\left(a_{1}, \ldots, a_{k}\right) .
$$

(b) Suppose that $\boldsymbol{v}: \mathbb{R}^{k} \rightarrow \mathbb{R}^{p}$ satisfies

$$
\nabla \cdot \mu(\boldsymbol{t})\left(\nabla \boldsymbol{v}+\boldsymbol{e}^{\infty}\right)=\mathbf{0}
$$

where $\mu(\boldsymbol{t})=(1-\chi(\boldsymbol{t})) \mu_{-}+\chi(\boldsymbol{t}) \mu_{+}$. Then

$$
e^{\infty}=e^{+}+\frac{\llbracket \mu \rrbracket}{\mu_{-}} e^{+}\langle\Gamma(\boldsymbol{n})\rangle_{a}
$$

where $\boldsymbol{e}^{+}=\nabla \boldsymbol{v}_{+}+\boldsymbol{e}^{\infty}$ is the strain field in the inclusion. Here

$$
\langle\Gamma(\boldsymbol{n})\rangle_{\boldsymbol{a}}=f_{\mathbb{S}^{k-1}} \Gamma\left(\boldsymbol{a}^{-1} \boldsymbol{n}\right) d S(\boldsymbol{n}), \quad \Gamma(\boldsymbol{\omega})=\frac{\boldsymbol{\omega} \otimes \boldsymbol{\omega}}{|\boldsymbol{\omega}|^{2}} .
$$

The proof of part (a) can be found in [73]. Part (b) is proved in exactly the same way (with simpler calculations). In particular, the explicit formulas for the solution shows that both $\boldsymbol{\psi}_{0}(\boldsymbol{t})$ and $\boldsymbol{\psi}^{\prime}(\boldsymbol{t})$ are bounded and $\nabla \boldsymbol{\psi}_{0} \in L^{2}\left(\mathbb{R}^{k} ; \mathbb{R}^{k \times k}\right)$ and $\nabla \boldsymbol{\psi}^{\prime} \in L^{2}\left(\mathbb{R}^{k} ; \mathbb{R}^{d-k \times k}\right)$. Hence, the corresponding test function $\phi \in \overline{\mathcal{C}}_{k}$, and our general theory applies.

Remark 5.3. The tensor $\left\langle\mathrm{K}_{\mathrm{C}_{-}}(\boldsymbol{n})\right\rangle_{\boldsymbol{a}}$ has the property that

$$
\mathrm{S}=\left\langle\mathrm{K}_{\mathrm{C}_{-}}(\boldsymbol{n})\right\rangle_{\boldsymbol{a}} \mathrm{C}_{-}
$$

is the Eshelby tensor [36] for the ellipsoidal inhomogeneity relating the eigenstrain $\varepsilon^{*}=\llbracket \mathrm{C}^{-1} \rrbracket \boldsymbol{\sigma}^{+}$and the inhomogeneity strain $\varepsilon^{d}=\varepsilon^{+}-\varepsilon^{\infty}$.

Theorem 5.2 provides a relation between the strain at infinity and the uniform field in the inclusion. For instance, the explicit Fourier space representation of the field in the exterior of the inclusion can be written as

$$
\widehat{e(\boldsymbol{u})}(\boldsymbol{\omega})=-\widehat{\chi}(\boldsymbol{\omega}) \mathrm{K}_{\mathrm{C}_{-}}(\boldsymbol{\omega}) \llbracket C \rrbracket \varepsilon^{+}, \quad \widehat{\nabla \boldsymbol{v}}(\boldsymbol{\omega})=-\frac{\llbracket \mu \rrbracket}{\mu_{-}} \widehat{\chi}(\boldsymbol{\omega}) e^{+} \Gamma(\boldsymbol{\omega}) .
$$

For our purposes, however, we would only need the relations (5.5) and (5.6).

\subsubsection{Noether-Eshelby equations}

In this problem the Noether-Eshelby equation provides additional conditions only at the discontinuities of $\nabla \phi$ and reduces to the Maxwell relation (4.21). In [70] Kublanov and Freidin studied the ellipsoidal inclusions in $3 D$ space that satisfy the Maxwell condition, where they also computed the Eshelby tensor explicitly for such ellipsoids. Here we generalize some of their results to elliptical cylinders with arbitrary dimension $k$ of cross-section in $\mathbb{R}^{d}$.

In [49] we have shown that the Maxwell relation for the energy (5.1) takes the form

$$
\llbracket w \rrbracket+\frac{1}{2}\left(\llbracket C \rrbracket \varepsilon_{+}, \varepsilon_{+}\right)+\frac{1}{2}\left(\mathrm{~K}_{\mathrm{C}_{-}}(\boldsymbol{n}) \boldsymbol{q}_{+}, \boldsymbol{q}_{+}\right)=0,
$$


where $\boldsymbol{q}_{+}=-\llbracket C \rrbracket \varepsilon_{+}$and $\boldsymbol{n}$ is the outward unit normal on the boundary of the inclusion. For isotropic materials $C_{ \pm}$we can choose the coordinate axes aligned with the ellipsoid's principal directions and the generators of the elliptical cylinder. In that case the normal $\boldsymbol{n}$ has the last $d-k$ components equal to zero, while the first $k$ components form an element of the unit sphere $\mathbb{S}^{k-1}$. We will denote this $\boldsymbol{n} \in \mathbb{S}^{k-1}$ for short. It will be convenient to write the matrix $\boldsymbol{q}_{+}$in the block form

$$
\boldsymbol{q}_{+}=\left[\begin{array}{cc}
\boldsymbol{q}_{0} & \boldsymbol{p}^{T} \\
\boldsymbol{p} & \boldsymbol{q}^{\prime}
\end{array}\right]
$$

where $\boldsymbol{q}_{0}$ is $k \times k, \boldsymbol{p}$ is $(d-k) \times k$ and $\boldsymbol{q}^{\prime}$ is $(d-k) \times(d-k)$. Hence, the Maxwell relation (5.7) becomes

$$
\frac{\left|\boldsymbol{q}_{0} \boldsymbol{n}\right|^{2}+|\boldsymbol{p} \boldsymbol{n}|^{2}}{\mu_{-}}-\frac{\left(\lambda_{-}+\mu_{-}\right)\left(\boldsymbol{q}_{0} \boldsymbol{n}, \boldsymbol{n}\right)^{2}}{\mu_{-}\left(\lambda_{-}+2 \mu_{-}\right)}+\llbracket \lambda \rrbracket\left(\operatorname{Tr} \varepsilon_{+}\right)^{2}+2 \llbracket \mu \rrbracket\left|\varepsilon_{+}\right|^{2}+2 \llbracket w \rrbracket=0
$$

for all $\boldsymbol{n} \in \mathbb{S}^{k-1}$.

LEMMA 5.4. Let $\alpha \neq 0$. The function

$$
f(\boldsymbol{n})=\left|\boldsymbol{q}_{0} \boldsymbol{n}\right|^{2}+|\boldsymbol{p} \boldsymbol{n}|^{2}-\alpha\left(\boldsymbol{q}_{0} \boldsymbol{n}, \boldsymbol{n}\right)^{2}
$$

is constant on $\mathbb{S}^{k-1}$ if and only if $\boldsymbol{q}_{0}=q_{0} \boldsymbol{I}_{k}$ and $\boldsymbol{p}^{T} \boldsymbol{p}=p_{0}^{2} \boldsymbol{I}_{k}$. In particular, this implies that $\boldsymbol{p}=\mathbf{0}$, if $k>d / 2$.

Proof. Let

$$
f(\boldsymbol{n})=f_{1}(\boldsymbol{n})+f_{2}(\boldsymbol{n})-\alpha f_{3}(\boldsymbol{n}),
$$

where

$$
f_{1}(\boldsymbol{n})=\left(\boldsymbol{q}_{0}^{2} \boldsymbol{n}, \boldsymbol{n}\right), \quad f_{2}(\boldsymbol{n})=\left(\boldsymbol{p}^{T} \boldsymbol{p} \boldsymbol{n}, \boldsymbol{n}\right), \quad f_{3}(\boldsymbol{n})=\left(\boldsymbol{q}_{0} \boldsymbol{n}, \boldsymbol{n}\right)^{2} .
$$

The function $f(\boldsymbol{n})$ is constant on the sphere $\mathbb{S}^{k-1}$ if and only if its differential is zero on at any $\boldsymbol{n} \in \mathbb{S}^{k-1}$. Let $\boldsymbol{n}_{0}$ be an eigenvector of $\boldsymbol{q}_{0}$ (and therefore of $\boldsymbol{q}_{0}^{2}$ ). Then $d f_{1}\left(\boldsymbol{n}_{0}\right)=d f_{3}\left(\boldsymbol{n}_{0}\right)=0$. Hence, we must have $d f_{3}\left(\boldsymbol{n}_{0}\right)=0$. Therefore, $\boldsymbol{n}_{0}$ must be an eigenvector of $\boldsymbol{p}^{T} \boldsymbol{p}$. Hence, $\boldsymbol{q}_{0}$ and $\boldsymbol{p}^{T} \boldsymbol{p}$ have a common orthonormal eigen-basis. Suppose, $\boldsymbol{n}_{1}$ and $\boldsymbol{n}_{2}$ are orthogonal unit eigenvectors corresponding to the eigenvalues $\nu_{1}$ and $\nu_{2}$ of $\boldsymbol{q}_{0}$ and eigenvalues $\tau_{1}$ and $\tau_{2}$ of $\boldsymbol{p}^{T} \boldsymbol{p}$. Let $\phi(\theta)=f\left(\boldsymbol{n}_{1} \cos \theta+\boldsymbol{n}_{2} \sin \theta\right)$. We compute

$$
\phi(\theta)=\left(\nu_{1}^{2}+\tau_{1}\right) \cos ^{2} \theta+\left(\nu_{2}+\tau_{2}\right) \sin ^{2} \theta-\alpha\left(\nu_{1} \cos ^{2} \theta+\nu_{2} \sin ^{2} \theta\right)^{2} .
$$

Now it is easy to see that $\phi(\theta)$ is constant if and only if $\nu_{1}=\nu_{2}$ and $\tau_{1}=\tau_{2}$. We conclude that both $\boldsymbol{q}_{0}$ and $\boldsymbol{p}^{T} \boldsymbol{p}$ must be multiples of the identity, since the pair of eigenvectors was chosen arbitrarily. Conversely, if $\boldsymbol{q}_{0}=q_{0} \boldsymbol{I}_{k}$ and $\boldsymbol{p}^{T} \boldsymbol{p}=p_{0}^{2} \boldsymbol{I}_{k}$ then $f(\boldsymbol{n})=(1-\alpha) q_{0}^{2}+p_{0}^{2}$.

The assumption (5.2) ensures applicability of the lemma to (5.8). We conclude that

$$
\boldsymbol{\varepsilon}_{+}=\left[\begin{array}{cc}
e\left(\boldsymbol{\psi}_{0}^{+}\right)+\boldsymbol{\varepsilon}_{0} & \frac{1}{2}\left(\nabla \boldsymbol{\psi}_{+}^{\prime}\right)^{T}+\boldsymbol{E}^{T} \\
\frac{1}{2} \nabla \boldsymbol{\psi}_{+}^{\prime}+\boldsymbol{E} & \boldsymbol{\varepsilon}^{\prime}
\end{array}\right]=\left[\begin{array}{cc}
\varepsilon_{0}^{+} \boldsymbol{I}_{k} & \boldsymbol{E}_{+}^{T} \\
\boldsymbol{E}_{+} & \boldsymbol{\varepsilon}_{+}^{\prime}
\end{array}\right], \quad \boldsymbol{E}_{+}^{T} \boldsymbol{E}_{+}=E_{0}^{2} \boldsymbol{I}_{k},
$$

where the scalars $\varepsilon_{0}^{+}$and $E_{0}$ satisfy the Maxwell relation

$$
\begin{aligned}
\frac{\left(\llbracket k \lambda+2 \mu \rrbracket \varepsilon_{0}^{+}+\llbracket \lambda \rrbracket \operatorname{Tr} \varepsilon^{\prime}\right)^{2}}{\lambda_{-}+2 \mu_{-}}+\llbracket \lambda \rrbracket\left(\varepsilon_{0}^{+} k+\operatorname{Tr} \varepsilon^{\prime}\right)^{2}+2 k \llbracket \mu \rrbracket\left(\varepsilon_{0}^{+}\right)^{2} & +\frac{4 \llbracket \mu \rrbracket\left(k \mu_{-}+\llbracket \mu \rrbracket\right)}{\mu_{-}} E_{0}^{2}+2 \llbracket \mu \rrbracket\left|\varepsilon^{\prime}\right|^{2}+2 \llbracket w \rrbracket=0 .
\end{aligned}
$$


Applying Theorem 5.2 to (5.3) and using (5.9) we obtain

$$
\left\{\begin{array}{l}
\varepsilon_{0}=\varepsilon_{0}^{+} \boldsymbol{I}_{k}+\frac{\varepsilon_{0}^{+} \llbracket k \lambda+2 \mu \rrbracket+\llbracket \lambda \rrbracket \operatorname{Tr} \boldsymbol{\varepsilon}^{\prime}}{\lambda_{-}+2 \mu_{-}}\langle\Gamma(\boldsymbol{n})\rangle_{\boldsymbol{a}}, \\
\boldsymbol{E}=\boldsymbol{E}_{+}\left(\boldsymbol{I}_{k}+\frac{\llbracket \mu \rrbracket}{\mu_{-}}\langle\Gamma(\boldsymbol{n})\rangle_{\boldsymbol{a}}\right) .
\end{array}\right.
$$

We note that the explicit expressions for $\langle\Gamma(\boldsymbol{n})\rangle_{\boldsymbol{a}}$ is available for $k=1\left(\langle\Gamma(\boldsymbol{n})\rangle_{\boldsymbol{a}}=1\right), k=2\left(\langle\Gamma(\boldsymbol{n})\rangle_{\boldsymbol{a}}=\right.$ $\operatorname{cof}(\boldsymbol{a}) / \operatorname{Tr} \boldsymbol{a})$ and $k=3$, when it can be expressed in terms of the elliptic integrals. However, we do not need to know $\langle\Gamma(\boldsymbol{n})\rangle_{\boldsymbol{a}}$ explicitly, we only need the set of diagonal matrices

$$
\mathcal{G}=\left\{\langle\Gamma(\boldsymbol{n})\rangle_{\boldsymbol{a}}: \boldsymbol{a}=\operatorname{diag}\left(a_{1}, \ldots, a_{k}\right), a_{i}>0, i=1, \ldots, k\right\} .
$$

Lemma 5.5.

$$
\mathcal{G}=\left\{\boldsymbol{A}=\operatorname{diag}\left(A_{1}, \ldots, A_{k}\right), A_{i}>0, i=1, \ldots, k, \operatorname{Tr} \boldsymbol{A}=1\right\} .
$$

The proof of the lemma is in the Appendix G.

We now apply Lemma 5.5 by taking the trace of the first equation in (5.11). We obtain

$$
\varepsilon_{0}^{+}=\frac{\left(\lambda_{-}+2 \mu_{-}\right) \operatorname{Tr} \varepsilon_{0}-\llbracket \lambda \rrbracket \operatorname{Tr} \varepsilon^{\prime}}{k \lambda_{+}+2 k \mu_{-}+2 \llbracket \mu \rrbracket} .
$$

The denominator in (5.12) is positive if either $\llbracket \lambda \rrbracket>0$ or $\llbracket \mu \rrbracket<0$. It could change sign if $\llbracket \lambda \rrbracket<0$ and $\llbracket \mu \rrbracket>0$. We therefore place the material with larger $\lambda$ or smaller $\mu$ inside the inclusion. In the well-ordered case $\left(\lambda_{+}>\lambda_{-}, \mu_{+}>\mu_{-}\right)$, either material can be placed inside the inclusion, while in the non well-ordered case only the material with larger $\lambda$ and smaller $\mu$ can be placed inside.

\subsubsection{Optimal orientation}

In our example the optimal orientation equation (4.13) becomes

$$
\boldsymbol{Q} \int_{\mathbb{R}^{k}}\left(\mathrm{C}(\boldsymbol{t})\left(\varepsilon^{\infty}+e(\phi)\right)-\mathrm{C}_{-} \varepsilon^{\infty}\right) \nabla \boldsymbol{\psi} d \boldsymbol{t}=\mathbf{0}
$$

We can rewrite the left-hand side in (5.13) as the sum of two terms $T_{1}$ and $T_{2}$

$$
T_{1}=\boldsymbol{Q} \int_{\mathbb{R}^{k}} \chi(\boldsymbol{t})\left(\llbracket C \rrbracket \varepsilon^{\infty}\right) \nabla \boldsymbol{\psi} d \boldsymbol{t}, \quad T_{2}=\boldsymbol{Q} \int_{\mathbb{R}^{k}}(\mathrm{C}(\boldsymbol{t}) e(\boldsymbol{\phi})) \nabla \boldsymbol{\psi} d \boldsymbol{t} .
$$

We compute

$$
\begin{gathered}
T_{1}=\left(2 \llbracket \mu \rrbracket \boldsymbol{E} \nabla \boldsymbol{\psi}_{0}^{+}+\llbracket \lambda \rrbracket\left(\operatorname{Tr} \boldsymbol{\varepsilon}_{0}+\operatorname{Tr} \boldsymbol{\varepsilon}^{\prime}\right) \nabla \boldsymbol{\psi}_{+}^{\prime}+2 \llbracket \mu \rrbracket \boldsymbol{\varepsilon}^{\prime} \nabla \boldsymbol{\psi}_{+}^{\prime}\right) \int_{\mathbb{R}^{k}} \chi(\boldsymbol{t}) d \boldsymbol{t} . \\
T_{2}=\int_{\mathbb{R}^{k}}\left\{\mu(\boldsymbol{t}) \nabla \boldsymbol{\psi}^{\prime} \nabla \boldsymbol{\psi}_{0}+\lambda(\boldsymbol{t})\left(\nabla \cdot \boldsymbol{\psi}_{0}\right) \nabla \boldsymbol{\psi}^{\prime}\right\} d \boldsymbol{t} .
\end{gathered}
$$

Using integration by parts we can rewrite $T_{2}$ as

$$
T_{2}=-\int_{\mathbb{R}^{k}} \boldsymbol{\psi}^{\prime} \otimes \nabla \cdot\left(\mathrm{C}(\boldsymbol{t}) e\left(\boldsymbol{\psi}_{0}\right)\right) d \boldsymbol{t}+\int_{\mathbb{R}^{k}} \nabla \cdot\left(\mu(\boldsymbol{t}) \nabla \boldsymbol{\psi}^{\prime}\right) \otimes \boldsymbol{\psi}_{0} d \boldsymbol{t} .
$$

Using equations (5.3) we get

$$
\nabla \cdot\left(\mathrm{C}(\boldsymbol{t}) e\left(\boldsymbol{\psi}_{0}\right)\right)=-\nabla \cdot\left(\chi(\boldsymbol{t}) \llbracket C \rrbracket \widehat{\varepsilon}_{0}\right), \quad \nabla \cdot\left(\mu(\boldsymbol{t}) \nabla \boldsymbol{\psi}^{\prime}\right)=-2 \llbracket \mu \rrbracket \nabla \cdot(\chi(\boldsymbol{t}) \boldsymbol{E}),
$$

where

$$
\widehat{\varepsilon}_{0}=\varepsilon_{0}+\frac{\llbracket \lambda \rrbracket \operatorname{Tr} \varepsilon^{\prime}}{k \llbracket \lambda \rrbracket+2 \llbracket \mu \rrbracket} \boldsymbol{I}_{k}
$$


Thus, we obtain

$$
T_{2}=\int_{\mathbb{R}^{k}} \chi(\boldsymbol{t})\left\{-\nabla \boldsymbol{\psi}^{\prime}\left(\llbracket \mathbb{C} \rrbracket \widehat{\boldsymbol{\varepsilon}}_{0}\right)+2 \llbracket \mu \rrbracket \boldsymbol{E}\left(\nabla \boldsymbol{\psi}_{0}\right)^{T}\right\} d \boldsymbol{t} .
$$

Computing $\llbracket C \rrbracket \widehat{\varepsilon}_{0}$ and combining with $T_{1}$ we write (5.13) as

$$
\boldsymbol{E} e\left(\boldsymbol{\psi}_{0}^{+}\right)+\frac{1}{2} \varepsilon^{\prime} \nabla \boldsymbol{\psi}_{+}^{\prime}-\frac{1}{2} \nabla \boldsymbol{\psi}_{+}^{\prime} \varepsilon_{0}=\mathbf{0} .
$$

Substituting the values

$$
e\left(\boldsymbol{\psi}_{0}^{+}\right)=\varepsilon_{0}^{+} \boldsymbol{I}_{k}-\varepsilon_{0}, \quad \frac{1}{2} \nabla \boldsymbol{\psi}_{+}^{\prime}=\boldsymbol{E}_{+}-\boldsymbol{E},
$$

obtained from (5.9), into (5.14) we get

$$
\left(\varepsilon_{0}^{+} \boldsymbol{I}_{k}-\varepsilon^{\prime}\right) \boldsymbol{E}=\boldsymbol{E}_{+} \varepsilon_{0}-\boldsymbol{\varepsilon}^{\prime} \boldsymbol{E}_{+} .
$$

Substituting the second equation in (5.11) into (5.15) we obtain

$$
\frac{\llbracket \mu \rrbracket}{\mu_{-}}\left(\varepsilon_{0}^{+} \boldsymbol{I}_{k}-\boldsymbol{\varepsilon}^{\prime}\right) \boldsymbol{E}_{+}\langle\Gamma(\boldsymbol{n})\rangle_{\boldsymbol{a}}=\boldsymbol{E}_{+}\left(\boldsymbol{\varepsilon}_{0}-\varepsilon_{0}^{+} \boldsymbol{I}_{k}\right) .
$$

Applying the first equation in (5.11) and the invertibility of $\langle\Gamma(\boldsymbol{n})\rangle_{\boldsymbol{a}}$ we finally conclude

$$
\left[\left(\lambda_{-} \llbracket \mu \rrbracket-k \mu_{-} \llbracket \lambda \rrbracket\right) \varepsilon_{0}^{+}-\mu_{-} \llbracket \lambda \rrbracket \operatorname{Tr} \boldsymbol{\varepsilon}^{\prime}\right] \boldsymbol{E}_{+}=\left(\lambda_{-}+2 \mu_{-}\right) \llbracket \mu \rrbracket \varepsilon^{\prime} \boldsymbol{E}_{+} .
$$

The equations (5.9) and (5.16) say that, provided $E_{0} \neq 0$, the $k$ columns of the $(d-k) \times k$ matrix $\boldsymbol{E}_{+} / E_{0}$ are orthonormal eigenvectors of the $(d-k) \times(d-k)$ matrix $\varepsilon^{\prime}$. All of them correspond to the same eigenvalue

$$
\nu=\frac{\left(\lambda_{-} \llbracket \mu \rrbracket-k \mu_{-} \llbracket \lambda \rrbracket\right) \varepsilon_{0}^{+}-\mu_{-} \llbracket \lambda \rrbracket \operatorname{Tr} \varepsilon^{\prime}}{\left(\lambda_{-}+2 \mu_{-}\right) \llbracket \mu \rrbracket} .
$$

\subsubsection{Explicit bounds}

If $E_{0}=0$ then $\boldsymbol{E}_{+}=\mathbf{0}$ and the relation (5.16) is identically satisfied. In that case the equation for the sets $\mathfrak{N}_{k}^{\text {ell }}$ introduced in Definition 5.1 is provided by the relation (5.10), which becomes

$$
\frac{\left(\lambda_{-}+2 \mu_{-}\right)\left(\llbracket k \lambda+2 \mu \rrbracket \operatorname{Tr} \varepsilon_{0}+k \llbracket \lambda \rrbracket \operatorname{Tr} \varepsilon^{\prime}\right)^{2}}{\llbracket k \lambda+2 \mu \rrbracket\left(k \lambda_{+}+2 k \mu_{-}+2 \llbracket \mu \rrbracket\right)}+\frac{2 \llbracket \lambda \rrbracket \llbracket \mu \rrbracket\left(\operatorname{Tr} \varepsilon^{\prime}\right)^{2}}{\llbracket k \lambda+2 \mu \rrbracket}+2 \llbracket \mu \rrbracket\left|\varepsilon^{\prime}\right|^{2}+2 \llbracket w \rrbracket=0 .
$$

Equation (5.18) provides a characterization of the union of $\left(\begin{array}{l}d \\ k\end{array}\right)$ surfaces in the space of eigenvalues of $\varepsilon$. Different surfaces in this union are obtained by choosing $k$ of the $d$ eigenvalues of $\varepsilon$ forming the diagonal of the $k \times k$ diagonal matrix $\varepsilon_{0}$. Another union of $\left(\begin{array}{l}d \\ k\end{array}\right)$ surfaces are obtained by exchanging "+" and "-" subscripts in (5.18). The entire collection of $2\left(\begin{array}{l}d \\ k\end{array}\right)$ surfaces comprises the part of the set $\mathfrak{N}_{k}^{\text {ell }}$ corresponding to $E_{0}=0$.

If $E_{0} \neq 0$, then the optimality of orientation condition (5.16) requires $\varepsilon^{\prime}$ to have $k$ equal eigenvalues. Together with the relation (5.10) this places $\varepsilon$ on a co-dimension $k$ surface in $\operatorname{Sym}\left(\mathbb{R}^{d}\right)$. Such surfaces cannot be candidates for the binodal when $k>1$ and are therefore discarded, leaving only the case $k=1$. In this case $\langle\Gamma(\boldsymbol{n})\rangle_{\boldsymbol{a}}=1$ and the matrices $\boldsymbol{E}$ and $\boldsymbol{E}_{+}$are vectors in $\mathbb{R}^{d-1}$, related via (5.11)

$$
\boldsymbol{E}=\frac{\mu_{+}}{\mu_{-}} \boldsymbol{E}_{+} .
$$

If we choose one of the coordinate axes to be aligned with $\boldsymbol{E}$ then, according to (5.16), $\varepsilon$ must have the following structure

$$
\boldsymbol{\varepsilon}=\left[\begin{array}{ccc}
\varepsilon_{0} & \frac{\mu_{+}}{\mu_{-}} E_{0} & \mathbf{0} \\
\frac{\mu_{+}}{\mu_{-}} E_{0} & \nu & \mathbf{0} \\
\mathbf{0} & \mathbf{0} & \boldsymbol{\varepsilon}^{\prime \prime}
\end{array}\right]
$$


where $\nu$ is given by (5.17) with $k=1$. Writing it in terms of $\varepsilon_{0}$ and $\varepsilon^{\prime \prime}$, using (5.12) we obtain

$$
\nu=\frac{\left(\lambda_{-} \llbracket \mu \rrbracket-\mu_{-} \llbracket \lambda \rrbracket\right) \varepsilon_{0}-\mu_{+} \llbracket \lambda \rrbracket \operatorname{Tr} \varepsilon^{\prime \prime}}{\mu_{+} \llbracket \lambda+2 \mu \rrbracket+\lambda_{+} \llbracket \mu \rrbracket} .
$$

In order to write the equation for $\mathfrak{N}_{1}^{\text {ell }}$ in terms of the eigenvalues of $\varepsilon$ we introduce the notation

$$
\varepsilon_{1}=\left[\begin{array}{cc}
\varepsilon_{0} & \frac{\mu_{+}}{\mu_{-}} E_{0} \\
\frac{\mu_{+}}{\mu_{-}} E_{0} & \nu
\end{array}\right]
$$

The eigenvalues of $\varepsilon$ are split into two groups: the group of $d-2$ eigenvalues, comprising the diagonal of $\varepsilon^{\prime \prime}$, and the group containing the two eigenvalues of $\varepsilon_{1}$. It will be convenient to introduce variables

$$
X=\frac{\operatorname{Tr} \varepsilon_{1}}{\sqrt{2}}, \quad Y^{2}=\frac{1}{2}\left(\left(\operatorname{Tr} \varepsilon_{1}\right)^{2}-4 \operatorname{det} \varepsilon_{1}\right), \quad Z=\frac{\operatorname{Tr} \varepsilon^{\prime \prime}}{\sqrt{2}},
$$

which are well-known functions of the eigenvalues. Then the formula (5.12) becomes

$$
\varepsilon_{0}^{+}=\frac{\left(\mu_{-} \llbracket \lambda+2 \mu \rrbracket+\lambda_{-} \llbracket \mu \rrbracket\right) \varepsilon_{0}-\sqrt{2} \llbracket \lambda \rrbracket \llbracket \mu \rrbracket Z}{\mu_{+} \llbracket \lambda+2 \mu \rrbracket+\lambda_{+} \llbracket \mu \rrbracket} .
$$

From the equation $\varepsilon_{0}+\nu=\operatorname{Tr} \varepsilon_{1}$ we find

$$
\varepsilon_{0}=\frac{\left(\mu_{+} \llbracket \lambda+2 \mu \rrbracket+\lambda_{+} \llbracket \mu \rrbracket\right) X+\mu_{+} \llbracket \lambda \rrbracket Z}{\sqrt{2} \llbracket \mu \rrbracket\left(\lambda_{+}+\mu_{+}\right)} .
$$

We also have

$$
2 E_{0}^{2}=\left(\frac{\mu_{-}}{\mu_{+}}\right)^{2}\left(Y^{2}-\left(\mu_{+} \frac{\llbracket \lambda+\mu \rrbracket X+\llbracket \lambda \rrbracket Z}{\llbracket \mu \rrbracket\left(\lambda_{+}+\mu_{+}\right)}\right)^{2}\right) .
$$

If we now substitute (5.21), (5.22) and (5.24) into (5.10), taking into account (5.23), we obtain a representation for the $E_{0} \neq 0$ part of $\mathfrak{N}_{1}^{\text {ell }}$ in terms of the eigenvalues of $\varepsilon$ :

$$
\frac{\left(\lambda_{-}+\mu_{-}\right) \llbracket \lambda+\mu \rrbracket}{\lambda_{+}+\mu_{+}} X^{2}+2 \frac{\left(\lambda_{-}+\mu_{-}\right) \llbracket \lambda \rrbracket}{\lambda_{+}+\mu_{+}} X Z+\frac{\llbracket \lambda \rrbracket\left(\lambda_{-}+\mu_{+}\right)}{\lambda_{+}+\mu_{+}} Z^{2}+\frac{\llbracket \mu \rrbracket \mu_{-}}{\mu_{+}} Y^{2}+\llbracket \mu \rrbracket\left|\varepsilon^{\prime \prime}\right|^{2}=-\llbracket w \rrbracket .
$$

We interpret (5.25) as the union of $\left(\begin{array}{l}d \\ 2\end{array}\right)$ surfaces. Each of these surfaces is characterized by two (out of $d$ ) eigenvalues corresponding to $\varepsilon_{1}$. Another union of $\left(\begin{array}{l}d \\ 2\end{array}\right)$ surfaces is obtained from (5.25) by interchanging "+" and "-" subscripts in the well-ordered case. The entire collection of $2\left(\begin{array}{l}d \\ 2\end{array}\right)$ surfaces comprises the part of the set $\mathfrak{N}_{1}^{\text {ell }}$ corresponding to $E_{0} \neq 0$.

When $k>1$ (and hence $E_{0}=0$ ), we have only used the fact that $\operatorname{Tr}\langle\Gamma(\boldsymbol{n})\rangle_{\boldsymbol{a}}=1$. The positive definiteness of $\langle\Gamma(\boldsymbol{n})\rangle_{\boldsymbol{a}}$ gives us the validity domain for the equation (5.18). Substituting (5.12) into (5.11) and solving for $\langle\Gamma(\boldsymbol{n})\rangle_{\boldsymbol{a}}$ we obtain

$$
\frac{\left(\lambda_{-}+2 \mu_{-}\right) \boldsymbol{I}_{k}}{k \lambda_{+}+2 k \mu_{-}+2 \llbracket \mu \rrbracket} \leq \frac{\llbracket k \lambda+2 \mu \rrbracket \varepsilon_{0}+\llbracket \lambda \rrbracket\left(\operatorname{Tr} \varepsilon^{\prime}\right) \boldsymbol{I}_{k}}{\llbracket k \lambda+2 \mu \rrbracket \operatorname{Tr} \varepsilon_{0}+k \llbracket \lambda \rrbracket \operatorname{Tr} \boldsymbol{\varepsilon}^{\prime}} \leq \frac{\left(\lambda_{-}+2 \mu_{+}+k \llbracket \lambda \rrbracket\right) \boldsymbol{I}_{k}}{k \lambda_{+}+2 k \mu_{-}+2 \llbracket \mu \rrbracket} .
$$

This statement is equivalent to the inequalities $\mathbf{0} \leq\langle\Gamma(\boldsymbol{n})\rangle_{\boldsymbol{a}} \leq \boldsymbol{I}_{k}$ understood in the sense of quadratic forms.

It is easy to check that the upper bound in (5.26) is a consequence of the lower bound, due to the fact that

$$
\frac{\lambda_{-}+2 \mu_{-}}{k \lambda_{+}+2 k \mu_{-}+2 \llbracket \mu \rrbracket}>0,
$$


and we conclude that for $k>1(5.18)$ is the equation of $\mathfrak{N}_{k}^{\text {ell }}$, provided

$$
\frac{\llbracket k \lambda+2 \mu \rrbracket \varepsilon_{0}+\llbracket \lambda \rrbracket\left(\operatorname{Tr} \boldsymbol{\varepsilon}^{\prime}\right) \boldsymbol{I}_{k}}{\llbracket k \lambda+2 \mu \rrbracket \operatorname{Tr} \varepsilon_{0}+k \llbracket \lambda \rrbracket \operatorname{Tr} \boldsymbol{\varepsilon}^{\prime}} \geq \frac{\left(\lambda_{-}+2 \mu_{-}\right) \boldsymbol{I}_{k}}{k \lambda_{+}+2 k \mu_{-}+2 \llbracket \mu \rrbracket}
$$

in the sense of quadratic forms. Equation (5.18) and inequality (5.27) reduce to the results of Kublanov and Freidin [70], when $k=d=3$.

If the materials are well-ordered, we may interchange the materials (i.e. consider an inclusion of phase "-" in the matrix of phase "+"). In that case the inequalities in (5.26) and (5.27) are reversed, while the subscripts "+" and "-" are interchanged.

Notice that when $k=1$ we have $\langle\Gamma(\boldsymbol{n})\rangle_{\boldsymbol{a}}=1$ and there are no extra inequalities in the case $E_{0}=0$. However, when $E_{0} \neq 0$, equation (5.24) implies that the values of the variables $(X, Y, Z)$ must satisfy

$$
|Y| \geq \mu_{+}\left|\frac{\llbracket \lambda+\mu \rrbracket X+\llbracket \lambda \rrbracket Z}{\llbracket \mu \rrbracket\left(\lambda_{+}+\mu_{+}\right)}\right|
$$

This is the range of the validity of equation (5.25).

Let us verify that all solutions to (4.12) satisfy the non-degeneracy condition (4.26) of Corollary 4.7. An easy calculation shows that

$$
W_{\varepsilon}^{\circ}(\varepsilon, e(\boldsymbol{\psi}))=\llbracket C \rrbracket \varepsilon^{+} \chi(\boldsymbol{t}) .
$$

Hence, $\partial J_{k}(\boldsymbol{F}, \boldsymbol{\phi}) / \partial \boldsymbol{F}=\mathbf{0}$ if and only if $\boldsymbol{\varepsilon}^{+}=\mathbf{0}$. However, $\boldsymbol{\varepsilon}^{+}=\mathbf{0}$ contradicts (5.10).

Remark 5.6. It will be shown in [49] that the surface patch $\mathfrak{N}_{2}^{\text {ell }}$ given by (5.18), (5.26) is indeed a part of the binodal when $d=2$. If we choose $\boldsymbol{F} \in \mathfrak{N}_{2}^{\text {ell }}$ and the corresponding elliptical inclusion, then the field $\varepsilon^{\infty}+e(\phi)$ will stay strictly away from the singular boundaries of the quadratic energy wells. Hence, if we choose $\widetilde{\boldsymbol{F}} \notin \overline{\mathfrak{B}}$ sufficiently close to $\boldsymbol{F}$ and solve (5.30) then the solution will also be a nontrivial solution of (4.12) $)_{1}$, with $W(\boldsymbol{F})$ given by (5.1). Therefore, the "bifurcation" in (4.12)1 alone is not sufficient to obtain any bounds on the binodal region.

\subsection{Interacting cylindrical inclusions}

In this section we give an example of the test field $\phi \in \mathcal{C}_{1} \backslash \overline{\mathcal{C}}_{1}$ satisfying (4.12)-(4.13). More specifically, we construct a 1-parameter family of energetically equivalent $\mathcal{C}_{1}$ test fields interpolating between the $\mathcal{C}_{2}$ test fields (corresponding to elliptical inclusions) and the rank-two laminates discussed in Section 3.2.3.

\subsubsection{Euler-Lagrange equations}

We are looking for equilibrium configurations where the materials "+" and "-" occupy complementary subdomains $\Omega_{+}$and $\Omega_{-}$that are periodic with period 1 in $y$-direction. We further assume that the material "+" occupies a compact subset in the fundamental region $Y_{1}=\mathbb{R} \times[-1 / 2,1 / 2]$ with smooth boundary $\Sigma$. The first equation in (4.12) is

$$
\nabla \cdot(\mathrm{C}(\boldsymbol{x}) e(\boldsymbol{u}))=\mathbf{0}, \quad \boldsymbol{x} \in \mathbb{R}^{2},
$$

understood in the sense of distributions. Here

$$
e(\boldsymbol{u})_{i j}=\frac{1}{2}\left(\frac{\partial u_{i}}{\partial x_{j}}+\frac{\partial u_{j}}{\partial x_{i}}\right)
$$

The equation (5.30) can be written as the standard Lamé system in $\Omega_{ \pm}$together with the interface conditions

$$
\llbracket \boldsymbol{u} \rrbracket=\mathbf{0}, \quad \llbracket C e(\boldsymbol{u}) \rrbracket \boldsymbol{n}=\mathbf{0}, \quad \boldsymbol{x} \in \Sigma .
$$

The method of complex potentials [91] allows one to characterize the set of solutions to (5.30)-(5.31) completely in $2 \mathrm{D}$. Accordingly, the vector $\boldsymbol{u}=\left(u_{1}, u_{2}\right) \in \mathbb{R}^{2}$ is written as a complex number $u=u_{1}+i u_{2}$. If $\boldsymbol{u}$ solves the Lamé system then

$$
u(z)=A \phi(z)-B(\bar{\psi}(z)+z \bar{\Phi}(z))
$$


where

$$
\Phi(z)=\phi^{\prime}(z), \quad A=\frac{1}{\kappa}+\frac{1}{2 \mu}, \quad B=\frac{1}{2 \mu} .
$$

Also, any $2 \times 2$ matrix $\boldsymbol{M}$ can be written as a pair of complex numbers $\boldsymbol{M}=[p, q]$, according to the rule

$$
\boldsymbol{M}=\left[\begin{array}{cc}
p_{1} & -p_{2} \\
p_{2} & p_{1}
\end{array}\right]+\left[\begin{array}{cc}
q_{1} & q_{2} \\
q_{2} & -q_{1}
\end{array}\right],
$$

where $p=p_{1}+i p_{2}$ and $q=q_{1}+i q_{2}$. Then, the complex representation of the vector $\boldsymbol{M} \boldsymbol{v}, \boldsymbol{v} \in \mathbb{R}^{2}$, is $p v+q \bar{v}$, and

$$
\nabla \boldsymbol{u}=\left[\frac{\partial u}{\partial z}, \frac{\partial u}{\partial \bar{z}}\right]
$$

Applying (5.33) to (5.32) we obtain $\nabla \boldsymbol{u}=[A \Phi-B \bar{\Phi},-2 B \bar{\Pi}]$, where

$$
\Pi(z)=\frac{1}{2}\left(\Psi(z)+\bar{z} \Phi^{\prime}(z)\right), \quad \Psi(z)=\psi^{\prime}(z) .
$$

We also obtain

$$
e(\boldsymbol{u})=\left[\frac{\Re \mathfrak{e} \Phi(z)}{\kappa},-\frac{\bar{\Pi}(z)}{\mu}\right], \quad \boldsymbol{\sigma}=[2 \Re \mathfrak{e} \Phi(z),-2 \bar{\Pi}(z)] .
$$

The continuity of displacements $\llbracket \boldsymbol{u} \rrbracket=\mathbf{0}$ can be conveniently written in differential form, via the representation (5.32):

$$
\llbracket A \Phi-B \bar{\Phi} \rrbracket \dot{z}-2 \llbracket B \bar{\Pi} \rrbracket \dot{\bar{z}}=0,
$$

where $\dot{z}$ is the derivative of the parametrization $z(t)$ of the interface $\Gamma$. The continuity of tractions reads

$$
\llbracket \Re \mathfrak{e} \Phi \rrbracket \dot{z}+\llbracket \bar{\Pi} \rrbracket \dot{\bar{z}}=0,
$$

since the complex representation of the unit normal $\boldsymbol{n}$ is $-i \dot{z} /|\dot{z}|$. In terms of the variables $\dot{z}=|\dot{z}| e^{i \alpha}$, $\Phi(z)=X$ and $\Pi(z) e^{2 i \alpha}=Y$, the system (5.34)-(5.35) can be written as

$$
\left\{\begin{array}{l}
\llbracket A X-B \bar{X} \rrbracket-2 \llbracket B \bar{Y} \rrbracket=0, \\
\llbracket \Re \mathfrak{e} X \rrbracket+\llbracket \bar{Y} \rrbracket=0 .
\end{array}\right.
$$

\subsubsection{Noether-Eshelby equations}

Under the assumptions of the smoothness of the interfaces the second equation in (4.12) can be replaced by the Maxwell relation (4.21), as discussed in Section 4.2.1.

$$
\llbracket W \rrbracket-(\{\mathrm{C} e(\boldsymbol{u})\}, \llbracket e(\boldsymbol{u}) \rrbracket)=0, \quad \boldsymbol{x} \in \Sigma,
$$

which in the $X$ and $Y$ variables we can be written as

$$
\llbracket \frac{2}{\kappa} \rrbracket \Re \mathfrak{e}\left(X_{+}\right) \Re \mathfrak{e}\left(X_{-}\right)+\llbracket \frac{2}{\mu} \rrbracket \Re \mathfrak{e}\left(Y_{+} \bar{Y}_{-}\right)=\llbracket w \rrbracket .
$$

\subsubsection{Optimal orientation}

In addition, the necessary condition (4.11), though not computable by itself, implies an easily verifiable additional condition (4.22), as discussed in Section 4.2.1. It can be written in terms of $X, Y$ as

$$
\llbracket \Re \mathfrak{e} X \rrbracket \llbracket A X-B \bar{X} \rrbracket+2 \llbracket \Re \mathfrak{e} X \rrbracket \llbracket B \bar{Y} \rrbracket+\llbracket \bar{Y} \rrbracket \llbracket A \bar{X}-B X \rrbracket+2 \llbracket \bar{Y} \rrbracket \llbracket B \bar{Y} \rrbracket=0 .
$$


If we eliminate $\llbracket \bar{Y} \rrbracket$ and $\llbracket B \bar{Y} \rrbracket$ by means of (5.36) we obtain

$$
\llbracket \Re \mathfrak{e} X \rrbracket \llbracket(A+B) \mathfrak{I m} X \rrbracket=0 .
$$

Hence, there are two possibilities. Either $\llbracket \Re \mathfrak{e} X \rrbracket=0$, corresponding to $\llbracket \boldsymbol{\sigma} \rrbracket=\mathbf{0}$ or $\llbracket(A+B) \mathfrak{I m} X \rrbracket=0$, corresponding to $\boldsymbol{a}=\lambda \boldsymbol{n}$ and $\llbracket \boldsymbol{\sigma} \rrbracket=\beta \boldsymbol{n}^{\perp} \otimes \boldsymbol{n}^{\perp}$ for some scalars $\lambda$ and $\beta$.

Case $\llbracket \Re \mathfrak{e} X \rrbracket=0$. In this case we get

$$
\left\{\begin{array}{l}
\Re \mathfrak{e} X_{+}=\Re \mathfrak{e} X X_{-}=\Re \mathfrak{e} X, \\
Y_{+}=Y_{-}=\llbracket \frac{1}{\mu} \rrbracket^{-1}\left(\llbracket \frac{1}{\kappa} \rrbracket \Re \mathfrak{e} X-i \llbracket\left(\frac{1}{\kappa}+\frac{1}{\mu}\right) \mathfrak{I m} X \rrbracket\right), \\
\llbracket \frac{1}{\kappa} \rrbracket \llbracket \frac{1}{\kappa}+\frac{1}{\mu} \rrbracket(\Re \mathfrak{e} X)^{2}+\llbracket\left(\frac{1}{\kappa}+\frac{1}{\mu}\right) \mathfrak{I m} X \rrbracket^{2}=\frac{1}{2} \llbracket \frac{1}{\mu} \rrbracket \llbracket w \rrbracket .
\end{array}\right.
$$

Then, the function

$$
f(z)= \begin{cases}\Re \mathfrak{e} \Phi_{+}(z), & z \text { in }+ \text { region } \\ \Re \mathfrak{e} \Phi_{-}(z), & z \text { in }- \text { region }\end{cases}
$$

is bounded and harmonic on $\mathbb{R}^{2}$. Hence, it is a constant. We conclude that $\Phi_{ \pm}^{\prime}(z)=0$ and hence the functions $\mathfrak{I m} X_{ \pm}$, and therefore $Y_{ \pm}=\frac{1}{2} \Psi_{ \pm}(z) \dot{z} / \dot{\bar{z}}$ are constants on $\Sigma$. Hence, we obtain $\psi_{+}(z)=2 Y_{+} \bar{z}+\gamma_{+}$on $\Sigma$. By assumption region "+" contains a compact inclusion $D$ with smooth boundary. Then we must have $Y_{+}=0$, since

$$
0=\int_{\partial D} \psi_{+}(z) d z=4 i Y_{+}|D| .
$$

This contradicts (5.40).

Case $\llbracket(A+B) \mathfrak{I m} X \rrbracket=0$. Then the function

$$
f(z)= \begin{cases}\left(\frac{1}{\kappa_{+}}+\frac{1}{\mu_{+}}\right) \mathfrak{I m} \Phi_{+}(z), \quad z \text { in }+ \text { region } \\ \left(\frac{1}{\kappa_{-}}+\frac{1}{\mu_{-}}\right) \mathfrak{I m} \Phi_{-}(z), \quad z \text { in }- \text { region }\end{cases}
$$

must be both bounded and harmonic. Therefore, it is constant. Hence, $\Phi_{ \pm}(z)$ is constant in the " \pm " region. We can assume without loss of generality, that $\Phi_{-}(z)$ is a real constant. Hence, $\Phi_{+}(z)$ is also a real constant. It follows from (5.36) that $Y_{ \pm}$are constants on $\Sigma$. By assumption, region "+" contains a compact inclusion $D$, then we must have $Y_{+}=0$ due to (5.41). Hence, from (5.36) we get

$$
\Re \mathfrak{e} X_{+}=\frac{\kappa_{+}\left(\kappa_{-}+\mu_{-}\right)}{\kappa_{-}\left(\kappa_{+}+\mu_{-}\right)} \Re \mathfrak{e} X_{-},
$$

while

$$
X_{-}=\Re \mathfrak{e} \Phi_{-}(z)=\frac{1}{4} \operatorname{Tr} \boldsymbol{\sigma}_{\infty}=\frac{1}{2} \kappa_{-} \operatorname{Tr} \varepsilon_{\infty} .
$$

Substituting these relations into (5.38) we get

$$
\left(\operatorname{Tr} \varepsilon_{\infty}\right)^{2}=\frac{-2 \llbracket w \rrbracket\left(\mu_{-}+\kappa_{+}\right)}{\llbracket \kappa \rrbracket\left(\kappa_{-}+\mu_{-}\right)} .
$$

Hence, in terms of $\operatorname{Tr} \varepsilon_{\infty}$ we obtain

$$
\psi_{+}(z)=0, \quad \phi_{+}(z)=\frac{\kappa_{+}\left(\kappa_{-}+\mu_{-}\right) \operatorname{Tr} \varepsilon_{\infty}}{2\left(\kappa_{+}+\mu_{-}\right)} z, \quad \phi_{-}(z)=\frac{1}{2}\left(\operatorname{Tr} \varepsilon_{\infty}\right) \kappa_{-} z .
$$



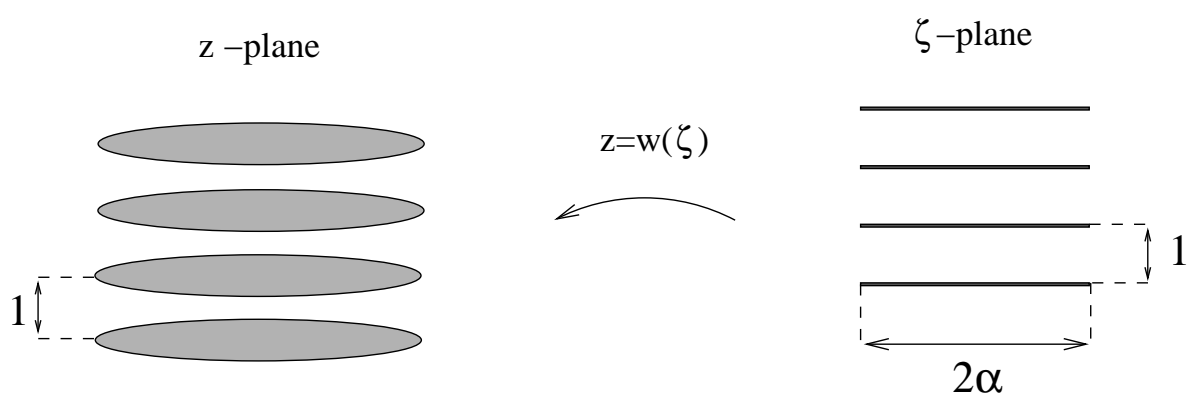

Figure 4: Conformal mapping of the exterior of the periodic array of slits onto the exterior of the periodic array of inclusions.

We also have

$$
\psi_{-}(z)=c \bar{z}+\gamma, \quad z \in \Sigma, \quad c=\frac{\mu_{-} \llbracket k \rrbracket \operatorname{Tr} \varepsilon_{\infty}}{\kappa_{+}+\mu_{-}} .
$$

The parameter $\gamma$ is locally constant on $\Sigma$ and can be chosen to be zero if $\Sigma$ is connected. Observe that the trivial solution $\boldsymbol{u}=\boldsymbol{\varepsilon}_{\infty} \boldsymbol{x}$ corresponds to the complex potentials

$$
\phi(z)=\frac{1}{2}\left(\operatorname{Tr} \varepsilon_{\infty}\right) \kappa_{-} z, \quad \psi(z)=b z, \quad b=\mu_{-}\left(\varepsilon_{\infty}^{(22)}-\varepsilon_{\infty}^{(11)}-2 i \varepsilon_{\infty}^{(12)}\right) .
$$

Hence, the function $p(z)=\psi_{-}(z)-b z$ must be $i$-periodic. Thus,

$$
\psi_{-}(z+i)=\psi_{-}(z)+b i
$$

It is now easy to verify that the as yet unused condition (4.13) holds automatically for any solution of the Lamé system satisfying (5.43). In fact, we have $\widehat{\boldsymbol{P}}^{*}(z)=0$ for all $z$.

\subsubsection{Optimal shapes}

We are now in a position to look for $i$-periodic structure of inclusions satisfying (5.43) and (5.44). The analysis here is an adaptation of the analysis in $[117,118,119,120,48]$ for the case of simply-periodic array of inclusions. Following [23, 48], we map the exterior of a periodic array of slits in the $\zeta$ plane conformally onto the region "-" in the $z$ plane (see Figure 4). More precisely, let $z=w(\zeta)$ map the $i$-periodic array of slits

$$
M_{n}=\{z=x+i n: x \in[-\alpha, \alpha]\}, \quad n \in \mathbb{Z}
$$

of length $2 \alpha$ (to be determined) in the $\zeta$ plane to the $i$-periodic array of inclusions with smooth boundary in the $z$ plane. The map $w$ must satisfy the following conditions:

$$
w(\zeta+i)=w(\zeta)+i
$$

At the endpoints of the slits $w(\zeta)=O\left(\sqrt{\zeta-\zeta_{n}}\right)$ as $\zeta \rightarrow \zeta_{n}$, where $\zeta_{n}= \pm \alpha+i n$ is an endpoint of the slit $M_{n}$, on account of the smoothness of the boundary of the inclusion in the $z$ plane.

Let us now substitute $z=w(\zeta)$ in $(5.44)$ and differentiate along the slit. Using notation $\Psi(\zeta)=\Psi-(w(\zeta))$ we obtain:

$$
\Psi(\zeta) w^{\prime}(\zeta)=c \overline{w^{\prime}}(\zeta), \quad \zeta \in M
$$

We can represent (5.47) using the following trick of Cherepanov [23]: Consider two analytic functions $F$ and $G$ chosen such that

$$
\begin{aligned}
& F^{\prime}(\zeta)=-\Psi(\zeta) w^{\prime}(\zeta)+c w^{\prime}(\zeta), \\
& G^{\prime}(\zeta)=-\Psi(\zeta) w^{\prime}(\zeta)-c w^{\prime}(\zeta) .
\end{aligned}
$$


Then (5.47) becomes

$$
\left.\begin{array}{l}
\Re \mathrm{e} F^{\prime}(\zeta)=0, \quad \zeta \in M_{n} \\
\Im \mathrm{m} G^{\prime}(\zeta)=0, \quad \zeta \in M_{n}
\end{array}\right\}
$$

Besides (5.50) the analytic functions $F$ and $G$ have the following properties: they are $i$-periodic, since both $\Psi(\zeta)$ and $w(\zeta)$ are, and at the endpoints $\zeta_{n}$ of the slits $M_{n}$

$$
F^{\prime}(\zeta)=O\left(\frac{1}{\sqrt{\zeta-\zeta_{n}}}\right) \text { and } G^{\prime}(\zeta)=O\left(\frac{1}{\sqrt{\zeta-\zeta_{n}}}\right) \text { as } \zeta \rightarrow \zeta_{n}
$$

also $F^{\prime}$ and $G^{\prime}$ are single valued and have no other singularities. Once such functions are found, using (5.48), (5.49) we can easily reconstruct $w(\zeta)$ and $\Psi(\zeta)$. The result is

$$
w(\zeta)=\frac{1}{2 c}(F(\zeta)-G(\zeta))+C_{0},
$$

where $C_{0}$ is an arbitrary constant of integration, and

$$
\Psi(\zeta)=-c \frac{F^{\prime}(\zeta)+G^{\prime}(\zeta)}{F^{\prime}(\zeta)-G^{\prime}(\zeta)}
$$

Now let's construct the functions $F$ and $G$. Consider the function [23]

$$
v(\zeta)=\sqrt{\frac{\cosh (2 \pi \zeta)-1}{\cosh (2 \pi \zeta)-\lambda}}, \quad \lambda=\cosh (2 \pi \alpha) .
$$

We claim that $v(\zeta)$ has the following properties:

1. $v(\zeta)$ is single valued analytic function in the exterior of the periodic array of slits $\left\{M_{n}: n \in \mathbb{Z}\right\}$;

2. $v(\zeta)$ is $i$-periodic;

3. $v(\zeta)=O\left(\frac{1}{\sqrt{\zeta-\zeta_{n}}}\right)$ as $\zeta \rightarrow \zeta_{n}$, and $v$ is bounded everywhere else;

4. $\Re \mathfrak{e}(v(\zeta))=0$ on $M_{n}$.

To justify the claim we choose the branch of the square root such that $\sqrt{1}=1$, with the branch cut along the negative real axis. Then the function $v(\zeta)$ has a branch cut wherever

$$
\frac{\cosh (2 \pi \zeta)-1}{\cosh (2 \pi \zeta)-\lambda}<0
$$

This is equivalent to the condition that $\cosh (2 \pi \zeta) \in(1, \lambda)$, which is satisfied only along the cuts $M_{n}$ (this is how the function (5.54) was constructed). Thus, properties 1 and 4 are proved. Property 2 follows from the $i$-periodicity of $\cosh (2 \pi \zeta)$. And property 3 follows from the fact that points $\zeta_{n}$ are simple points for $\cosh (2 \pi \zeta)$ (the derivative $2 \pi \sinh (2 \pi \alpha) \neq 0$ ).

We look for the functions $F^{\prime}$ and $G^{\prime}$ in the form

$$
\left\{\begin{array}{l}
F^{\prime}=r_{1} v(\zeta)+i d_{1} \\
G^{\prime}=i r_{2} v(\zeta)+d_{2}
\end{array}\right.
$$

where $r_{j}, d_{j} \in R$ are constants to be determined. It is easy to see that equations (5.50) are satisfied, as is the condition (5.51). In order to recover $F$ and $G$ from the above formulas we have to use the function

$$
V(\zeta)=\int_{i / 2}^{\zeta} v(z) d z .
$$


This function is single valued in the exterior of the periodic system of the slits because $\oint_{\Gamma_{R}} v(\zeta) d \zeta=0$, where $\Gamma_{R}$ is a rectangle with vertexes $\pm R \pm i / 2$. Indeed, the function $v(\zeta)$ is even and $i$-periodic. Therefore,

$$
\int_{-R}^{R} v(x-i / 2) d x=-\int_{R}^{-R} v(x+i / 2) d x, \quad i \int_{-1 / 2}^{1 / 2} v(R+i y) d y=-i \int_{1 / 2}^{-1 / 2} v(-R+i y) d y .
$$

The $i$-periodicity of $v(\zeta)$ implies that $V(\zeta+i)-V(\zeta)$ is independent of $\zeta$. Therefore,

$$
V(\zeta+i)-V(\zeta)=\lim _{R \rightarrow \infty} i \int_{-1 / 2}^{1 / 2} v(R+i y) d y=i .
$$

The periodicity condition (5.46) together with formulas (5.52), (5.56) and (5.58) implies

$$
d_{1}=r_{2}, \quad r_{1}-d_{2}=2 c .
$$

So that

Thus by $(5.53)$

$$
w(\zeta)=r V(\zeta)+(1-r) \zeta, \quad r=\frac{r_{1}-i r_{2}}{2 c} .
$$

$$
\psi_{2}(w(\zeta))=-c(\bar{r} V(\zeta)+(\bar{r}-1) \zeta)+\text { const. }
$$

Now using the translation law (5.45) for the potential $\psi_{-}$we obtain:

$$
r=\frac{1}{2}(1-\bar{q}), \quad q=\frac{b}{c} .
$$

We need to place further restriction on the value of parameter $r$ (i.e. on $\varepsilon_{\infty}$ ) so that the map $w(\zeta)$ given by (5.59) maps the exterior of the $i$-periodic array of slits $\left\{M_{n}: n \in \mathbb{Z}\right\}$ one-to-one and onto the exterior of the $i$-periodic array of inclusions $D_{n}=w\left(M_{n}\right)$. A necessary condition for univalence of $w(\zeta)$ is that $w^{\prime}(\zeta) \neq 0$. In other words $v(\zeta) \neq(r-1) / r$. The principal branch of the square root in (5.54) can take any value in the right half-plane $\Re \mathfrak{e} v \geq 0$. Hence, we require that $\Re \mathfrak{e}((r-1) / r)<0$. In other words $|r-1 / 2|<1 / 2$, or equivalently, $|q|<1$, i.e.

$$
\left|\varepsilon_{\infty}^{(22)}-\varepsilon_{\infty}^{(11)}-2 i \varepsilon_{\infty}^{(12)}\right|^{2}<-\frac{2 \llbracket w \rrbracket \llbracket \kappa \rrbracket}{\left(\kappa_{+}+\mu_{-}\right)\left(\kappa_{-}+\mu_{-}\right)} .
$$

It is easy to show that $|q|<1$ is also sufficient for univalence. Indeed, we only need to prove that $w\left(\zeta_{1}\right) \neq w\left(\zeta_{2}\right)$ for any $\zeta_{1} \neq \zeta_{2}$, such that $\mathfrak{I m}\left(\zeta_{j}\right) \in(0,1), j=1,2$. Observe that we can connect the points $\zeta_{1}$ and $\zeta_{2}$ by straight line without crossing any slits. Thus, we can write

$$
\frac{w\left(\zeta_{1}\right)-w\left(\zeta_{2}\right)}{\zeta_{1}-\zeta_{2}}=1-r+r \int_{0}^{1} v\left(t \zeta_{1}+(1-t) \zeta_{2}\right) d t
$$

If $w\left(\zeta_{1}\right)=w\left(\zeta_{2}\right)$ then we must have

$$
\int_{0}^{1} v\left(t \zeta_{1}+(1-t) \zeta_{2}\right) d t=\frac{r-1}{r} .
$$

However, the left-hand side is in the right half-plane $\Re \mathfrak{e}(v)>0$, while the right-hand side is in the left half-plane, when $|q|<1$. Thus, the map $w(\zeta)$ is univalent if and only if (5.62) holds.

The inequality (5.62) together with (5.42) describes a surface known to be in $\overline{\mathfrak{B}}$, since the non-degeneracy condition (4.26), that has the form (5.29) in our example, is obviously satisfied. The surface (5.42), (5.62) coincides with (5.18), (5.27) for $d=k=2$.

We compute that for any $\xi \in[-\alpha, \alpha]$

$$
V(\xi+0 i)=\frac{i}{\pi} \arccos \left(\frac{\cosh (\pi \xi)}{\cosh (\pi \alpha)}\right) .
$$




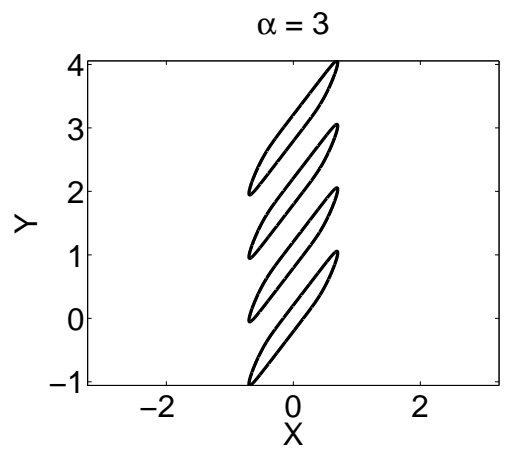

Figure 5: Periodic array of inclusions for $\alpha=3$ and $q=-0.4 \sqrt{2}(1+i)$.
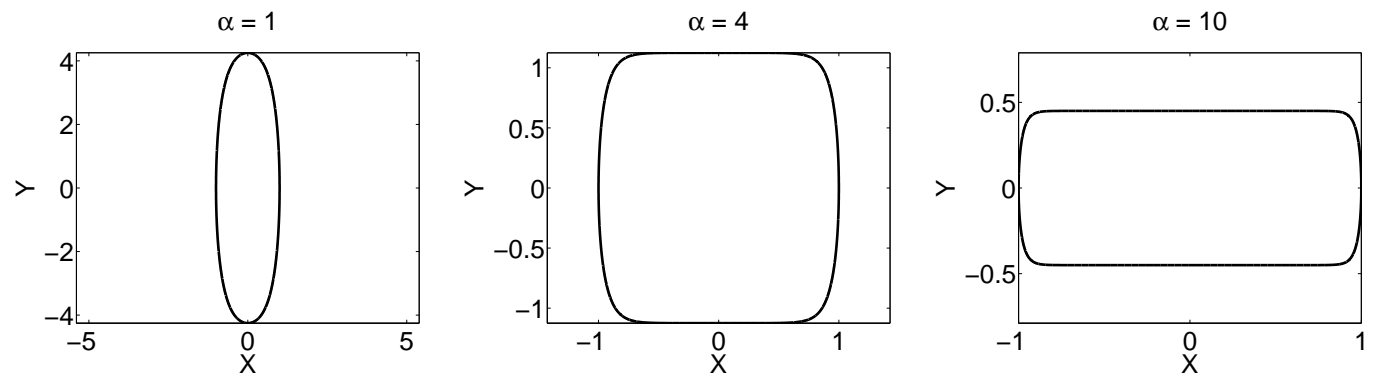

Figure 6: The shape of the component of a periodic array of inclusions for $q=-0.8$ and $\alpha=1,4$ and 10 .

Therefore the parametric equations of the upper half of the inclusion are

$$
x=\frac{1+q_{1}}{2} \xi-\frac{q_{2}}{2 i} V(\xi), \quad y=\frac{1-q_{1}}{2 i} V(\xi)-\frac{q_{2}}{2} \xi, \quad \xi \in[-\alpha, \alpha] .
$$

where $q=q_{1}+i q_{2}$. The parameter $\alpha$ is arbitrary. The structure for $\alpha=3$ and $q=-0.4 \sqrt{2}(1+i)$ is pictured in Figure 5.

Now, for simplicity let us examine in more detail the case when the periodic direction is chosen to be the eigendirection of $\varepsilon_{\infty}$. Then $b \in \mathbb{R}$, and hence $r \in(0,1)$. The parameter $\alpha>0$ can be chosen arbitrarily. The resulting shapes are different for different values of $\alpha$, yet all have the same energy. The equation of the upper half of the inclusion centered at the origin is

$$
y=\frac{1-q}{2 \pi} \arccos \left(\frac{\cosh \left(\frac{2 \pi x}{1+q}\right)}{\cosh (\pi \alpha)}\right), \quad x \in\left[-\frac{(1+q) \alpha}{2}, \frac{(1+q) \alpha}{2}\right] .
$$

When $\alpha \rightarrow 0$ the inclusions degenerate into the $i$-periodic array of small ellipses

$$
\frac{x^{2}}{(1+q)^{2}}+\frac{y^{2}}{(1-q)^{2}}=\frac{\alpha^{2}}{4}
$$

When $\alpha \rightarrow \infty$ the structure becomes a periodic array of horizontal layers of thickness $r$. However, for each large value $\alpha$ one needs to rescale the structure to keep the width of the inclusions constant, i.e. we change variables

$$
X=\frac{2 x}{(1+q) \alpha}, \quad Y=\frac{2 y}{(1+q) \alpha} .
$$


In the new variables the array of inclusions is $p$-periodic, where

$$
p=\frac{2 i}{(1+q) \alpha}
$$

is large, while the upper half of the inclusion centered at the origin has the equation

$$
Y=\frac{1}{\pi \alpha} \frac{1-q}{1+q} \arccos \left(\frac{\cosh (\pi \alpha X)}{\cosh (\pi \alpha)}\right), \quad X \in[-1,1] .
$$

When $\alpha \rightarrow \infty$ both the period $p$ and the vertical dimensions of the inclusions will go to zero and the structure will converge to a second rank laminate with inner volume fraction $r$, which is also known to permit detection of this part of the binodal.

Figure 6 shows the shapes of single inclusions given by (5.63) for $q=-0.8$ and $\alpha=1,4$ and 10. For each fixed value $\alpha$ the decay of the elastic fields along the $x$-direction is exponential. Therefore, the corresponding test function $\boldsymbol{\phi}=\boldsymbol{u}-\boldsymbol{\varepsilon}_{\infty} \boldsymbol{x}$ is in the space $\mathcal{C}_{1}$.

In summary, for each fixed value $\varepsilon_{\infty}$ satisfying (5.42) and (5.62), that are identical to (5.18), (5.27) for $d=k=2$, we found a 1-parameter family of $\mathcal{C}_{1}$ test fields satisfying (4.12)-(4.13) degenerating into $\mathcal{C}_{2}$ test fields (corresponding to elliptical inclusions) when $\alpha \rightarrow 0$ and to rank-two laminates when $\alpha \rightarrow \infty$. In other words, each member of the solution family identifies exactly the same marginally stable value of $\varepsilon_{\infty}$ as the simple elliptical inclusions, confirming previously obtained bounds. The isotropy and high nonconvexity of this example contributes to the abundance of rank-1 connected pairs $\boldsymbol{F}_{+}, \boldsymbol{F}_{-}$on the jump set [51], described in Section 4.2.2. This in turn provides sufficient flexibility for multiple structures to identify same marginally stable values of deformation gradients. For more general energies we expect fewer binodal points to be detectable through classical nucleation. For example in [47], essentially the same model with anisotropic tensors $C_{ \pm}$was considered in $2 d$. It was shown there that the regime analogous to (5.42), (5.62) can be detected only by second rank laminates, since the support of the optimal Young measure in (2.18) consists of specific three points which are inconsistent with classical nucleation.

\subsection{Laminates}

We have the following equations for $\varepsilon$ on a second rank lamination set. The "micro-level" system is

$$
\left\{\begin{array}{l}
\llbracket \varepsilon \rrbracket=b \odot \boldsymbol{m} \\
\llbracket C \varepsilon \rrbracket \boldsymbol{m}=\mathbf{0} \\
\llbracket C \varepsilon \rrbracket \boldsymbol{b}=\mathbf{0} \\
\llbracket w \rrbracket+\frac{1}{2}\left(\llbracket C \rrbracket \varepsilon_{ \pm}, \varepsilon_{ \pm}\right)=\mp \frac{1}{2}\left(C_{\mp} \llbracket \varepsilon \rrbracket, \llbracket \varepsilon \rrbracket\right) .
\end{array}\right.
$$

while the macro-level system is

$$
\left\{\begin{array}{l}
s \boldsymbol{\varepsilon}_{-}+(1-s) \boldsymbol{\varepsilon}_{+}-\boldsymbol{\varepsilon}=\boldsymbol{a} \odot \boldsymbol{n} \\
\left(s \mathrm{C}_{-} \boldsymbol{\varepsilon}_{-}+(1-s) \mathrm{C}_{+} \varepsilon_{+}-\mathrm{C}_{ \pm} \varepsilon\right) \boldsymbol{n}=\mathbf{0} \\
\left(s \mathrm{C}_{-} \boldsymbol{\varepsilon}_{-}+(1-s) \mathrm{C}_{+} \varepsilon_{+}-\mathrm{C}_{ \pm} \varepsilon\right) \boldsymbol{a}=\mathbf{0} \\
\llbracket w \rrbracket+\frac{1}{2}(\llbracket \mathrm{C} \rrbracket \varepsilon, \boldsymbol{\varepsilon})=\mp \frac{1}{2}\left(\mathrm{C}_{\mp} \boldsymbol{a} \odot \boldsymbol{n}, \boldsymbol{a} \odot \boldsymbol{n}\right),
\end{array}\right.
$$

where the upper sign corresponds to the situation when $\varepsilon$ lies in the "+" well, while the lower sign corresponds to the situation when $\varepsilon$ lies in the "-" well. The detailed analysis of this system of equations in 2D shows that by studying laminates one can confirm the bounds obtained in the analysis of classical nuclei and obtain new bounds inaccessible by the methods based exclusively on solving the associated PDE problem [49]. 


\section{Conclusions}

In this paper we systematically juxtaposed the conditions of marginal stability for weak and strong local minimizers in nonlinear elasticity. While the case of weak marginal stability, allowing one to determine the spinodal, can be studied in full detail, the case of strong marginal stability, bringing about the crucial notion of the binodal, is much less transparent. The reason is that binodal coincides with the boundary of the typically inscrutable quasiconvexity set.

It is essential that in order to locate the binodal one does not have to solve the difficult minimization problem for a non-convex integral functional of non-linear elasticity. Instead, one needs to deal with an equivalence class of parametric variational inequalities with a possibility that a particular formulation delivers a tractable characterization of the binodal. We used this freedom to obtain several characterizations of the binodal in terms of either PDEs or algebraic equations. In the former case the test functions are "well-behaved" members of a function space, in the latter they are weakly convergent sequences of gradients-generating laminate Young measures described by finitely many parameters.

We observe that our examples illustrate two types of nucleation patterns: isolated and interacting inclusions. Characterization of isolated inclusions reduces to solving a PDE problem in an infinite domain. To characterize interacting inclusions one needs to solve the same problem with periodic boundary conditions imposed at least in some directions. The most dramatic example of collective nucleation is provided by multi-rank laminates. In physical terms the periodicity of the boundary conditions means that the elastic interaction between individual inclusions is necessary for optimality and that we are dealing here with a cooperative phenomenon.

The variety of optimal patterns considered in this paper is in no way exhaustive which is exemplified by the concept of partially relaxed energy density introduced in this paper. This energy density is obtained by minimization with respect to a particular subclass of Young measures and can be used in the secondary nucleation PDE-based problem. The generalized bifurcations in the resulting PD can be interpreted as nucleation of composite precipitates that were left outside the scope of this paper (see, though, [97, 98, 99, $126,66,65])$.

Although we succeeded in building some conceptual links between the notions of spinodal and binodal, the ensuing thresholds remain fundamentally unconnected in the framework of classical nonlinear elasticity. The situation changes fundamentally if one considers regularized theory where the jumps of deformation gradients are replaced by smooth transition layers. In such settings (e.g. gradient theory, phase field theory, etc.) binodal and spinodal become parts of a single bifurcational diagram where the (regularized) spinodal indicates the actual bifurcation of a homogeneous configuration while the (regularized) binodal marks the transition between the trivial and the nontrivial branches of the global minimization path (see $[16,113]$ for 1D examples).

Several important issues have not been addressed in this paper. For instance, we did not attempt the differential characterization of the binodal (Clausius-Clapeyron type relations) and did not specifically study the nucleation conditions at the vertexes of the binodal associated with activation of essentially distinct nucleation mechanisms. Likewise, we had to exclude from our analysis material instabilities associated with internal and external surfaces. In the case of weak local minima the corresponding theory is rather well developed $[105,102,85]$ and the associated concept of surface spinodal is straightforward. For strong local minimizers, one needs to find the limits of the mysterious quasiconvexification-on-the-boundary set [14, 104, 102], making an explicit characterization of the surface binodal a formidable challenge. We leave all these issues for future studies.

\section{Acknowledgement}

This material is based upon work supported by the National Science Foundation under Grant No. 1008092 and the French ANR grant EVOCRIT (2008) . 


\section{A Proof of Lemma 3.3}

We note that $\mathcal{S}=W^{1, \infty}\left(\mathbb{R}^{d} ; \mathbb{R}^{m}\right) \cap \mathcal{S}_{0}$. It will be important to use the following embedding theorem for the space $\mathcal{S}_{0}$

Theorem A.1. Assume that $\phi \in \mathcal{S}_{0}$ and $d \geq 3$. Then there exists a unique constant $\boldsymbol{c} \in \mathbb{R}^{m}$ such that $\phi-c \in L^{\frac{2 d}{d-2}}\left(\mathbb{R}^{d} ; \mathbb{R}^{m}\right)$.

Proof. First, we remark that without loss of generality we may take $m=1$. Now recall the well-known potential theory operators. The Riesz transforms $R_{j}$ are defined by

$$
\mathcal{F}\left(R_{j} f\right)(\boldsymbol{\xi})=i \frac{\xi_{j}}{|\boldsymbol{\xi}|} \widehat{f}(\boldsymbol{\xi})
$$

where

$$
\mathcal{F}(f)(\boldsymbol{\xi})=\widehat{f}(\boldsymbol{\xi})=\int_{\mathbb{R}^{d}} f(\boldsymbol{x}) e^{2 \pi i(\boldsymbol{x}, \boldsymbol{\xi})} d \boldsymbol{x}
$$

is the Fourier transform. The operators $R_{j}$ map $L^{2}\left(\mathbb{R}^{d}\right)$ into $L^{2}\left(\mathbb{R}^{d}\right)$. The Riesz potential $I_{1}$ is defined by

$$
\mathcal{F}\left(I_{1} f\right)(\boldsymbol{\xi})=\frac{\widehat{f}(\boldsymbol{\xi})}{2 \pi|\boldsymbol{\xi}|} .
$$

It maps $L^{2}\left(\mathbb{R}^{d}\right)$ into $L^{\frac{2 d}{d-2}}\left(\mathbb{R}^{d}\right)$, [108].

If $\phi$ were smooth and compactly supported, we would have

$$
\phi=I_{1}\left(\sum_{j=1}^{d} R_{j}\left(\frac{\partial \phi}{\partial x_{j}}\right)\right) .
$$

Hence, we define

$$
\psi(\boldsymbol{x})=I_{1}\left(\sum_{j=1}^{d} R_{j}\left(g_{j}\right)\right),
$$

where $\boldsymbol{g}=\nabla \phi \in L^{2}\left(\mathbb{R}^{d} ; \mathbb{R}^{d}\right)$.

Let $\eta(\boldsymbol{x})$ be an arbitrary smooth compactly supported function. By definition of the distributional derivative we have

$$
\int_{\mathbb{R}^{d}}\left\{g_{k} \frac{\partial \eta}{\partial x_{j}}-g_{j} \frac{\partial \eta}{\partial x_{k}}\right\} d \boldsymbol{x}=-\left\langle\phi, \frac{\partial^{2} \eta}{\partial x_{k} \partial x_{j}}\right\rangle+\left\langle\phi, \frac{\partial^{2} \eta}{\partial x_{j} \partial x_{k}}\right\rangle=0 .
$$

By Plancherel's identity

$$
\int_{\mathbb{R}^{d}}\left(\widehat{g}_{k} \xi_{j}-\widehat{g}_{j} \xi_{k}\right) \overline{\hat{\eta}} d \boldsymbol{\xi}=0
$$

We conclude that

$$
\widehat{g}_{k}(\boldsymbol{\xi}) \xi_{j}=\widehat{g}_{j}(\boldsymbol{\xi}) \xi_{k}
$$

for a.e. $\boldsymbol{\xi} \in \mathbb{R}^{d}$. Thus,

$$
-2 \pi i \xi_{k} \widehat{\psi}(\boldsymbol{\xi})=\sum_{j=1}^{d} \frac{\xi_{k} \xi_{j} \widehat{g}_{j}(\boldsymbol{\xi})}{|\boldsymbol{\xi}|^{2}}=\widehat{g}_{k}(\boldsymbol{\xi}),
$$

due to (A.1). By Plancherel's identity

$$
\int_{\mathbb{R}^{d}} \psi(\boldsymbol{x}) \frac{\partial \eta}{\partial x_{k}} d \boldsymbol{x}=2 \pi i \int_{\mathbb{R}^{d}} \xi_{k} \widehat{\psi} \overline{\hat{\eta}} d \boldsymbol{\xi}=-\int_{\mathbb{R}^{d}} \widehat{g}_{k}(\boldsymbol{\xi}) \overline{\hat{\eta}} d \boldsymbol{\xi}=-\int_{\mathbb{R}^{d}} g_{k}(\boldsymbol{x}) \eta(\boldsymbol{x}) d \boldsymbol{x} .
$$

Therefore, $\nabla \psi=\boldsymbol{g}=\nabla \phi$ as distributions. The theorem is proved. 
The proof of Lemma 3.3 proceeds in different ways depending on the dimension $d$. If $d=1$, then

$$
\begin{gathered}
\frac{1}{R} \int_{R}^{2 R}|\phi|\left|\phi^{\prime}(x)\right| d x \leq \frac{\|\phi\|_{\infty}}{\sqrt{R}}\left(\int_{R}^{2 R}\left|\phi^{\prime}(x)\right|^{2} d x\right)^{1 / 2} \rightarrow 0, \text { as } R \rightarrow \infty . \\
\frac{1}{R^{2}} \int_{R}^{2 R}|\phi(x)|^{2} d x \leq \frac{\|\phi\|_{\infty}^{2}}{R} \rightarrow 0, \text { as } R \rightarrow \infty .
\end{gathered}
$$

Let $d=2$. We estimate

$$
\frac{1}{R} \int_{A_{R}}|\phi||\nabla \phi| d \boldsymbol{x} \leq\|\phi\|_{\infty} \sqrt{3 \pi}\left(\int_{A_{R}}|\nabla \phi|^{2} d \boldsymbol{x}\right)^{1 / 2} \rightarrow 0, \text { as } R \rightarrow \infty .
$$

By the Poincaré inequality

$$
\int_{A_{R}}\left|\boldsymbol{\phi}(\boldsymbol{x})-\langle\boldsymbol{\phi}\rangle_{A_{R}}\right|^{2} d \boldsymbol{x} \leq C_{0} R^{2} \int_{A_{R}}|\nabla \boldsymbol{\phi}|^{2} d \boldsymbol{x},
$$

where $C_{0}$ is the Poincaré constant for $A_{1}$. The boundedness of $\phi$ implies that there exists a sequence $R=R_{k}$ such that

$$
\lim _{k \rightarrow \infty}\langle\phi\rangle_{A_{R_{k}}}=\boldsymbol{c} .
$$

Hence, by the triangle inequality

$$
\left(\int_{A_{R}}|\boldsymbol{\phi}-\boldsymbol{c}|^{2} d \boldsymbol{x}\right)^{1 / 2} \leq\left(\int_{A_{R}}\left|\boldsymbol{\phi}-\langle\phi\rangle_{A_{R}}\right|^{2} d \boldsymbol{x}\right)^{1 / 2}+\left|A_{R}\right|^{1 / 2}\left|\langle\boldsymbol{\phi}\rangle_{A_{R}}-\boldsymbol{c}\right| .
$$

Then,

$$
\left(\frac{1}{R_{k}^{2}} \int_{A_{R_{k}}}|\boldsymbol{\phi}-\boldsymbol{c}|^{2} d \boldsymbol{x}\right)^{1 / 2} \leq\left(C_{0} \int_{A_{R_{k}}}|\nabla \phi|^{2} d \boldsymbol{x}\right)^{1 / 2}+\sqrt{3 \pi}\left|\langle\phi\rangle_{A_{R_{k}}}-\boldsymbol{c}\right| \rightarrow 0 \text { as } n \rightarrow \infty .
$$

Now assume that $d \geq 3$. By Theorem A.1 there exists a unique constant $\boldsymbol{c}$, such that $\boldsymbol{\phi}-\boldsymbol{c} \in L^{\frac{2 d}{d-2}}\left(\mathbb{R}^{d} ; \mathbb{R}^{m}\right)$. Using the inequality $a b \leq\left(a^{2}+b^{2}\right) / 2$ we get

$$
\frac{1}{R} \int_{A_{R}}|\boldsymbol{\phi}-\boldsymbol{c}||\nabla \boldsymbol{\phi}| d \boldsymbol{x}+\frac{1}{R^{2}} \int_{A_{R}}|\boldsymbol{\phi}-\boldsymbol{c}|^{2} d \boldsymbol{x} \leq \frac{1}{2} \int_{A_{R}}|\nabla \boldsymbol{\phi}|^{2} d \boldsymbol{x}+\frac{3}{2 R^{2}} \int_{A_{R}}|\boldsymbol{\phi}-\boldsymbol{c}|^{2} d \boldsymbol{x} .
$$

By Hölder inequality

$$
\frac{1}{R^{2}} \int_{A_{R}}|\phi-c|^{2} d \boldsymbol{x} \leq\left(\int_{A_{R}}|\boldsymbol{\phi}-\boldsymbol{c}|^{\frac{2 d}{d-2}} d \boldsymbol{x}\right)^{\frac{d-2}{d}} \rightarrow 0, \text { as } R \rightarrow \infty .
$$

The lemma is proved.

\section{B Proof of Lemma 3.7}

Let us begin with a technical lemma.

Lemma B.1. Suppose $\alpha(R)>0$ is such that $\alpha(R) \rightarrow 0$, as $R \rightarrow \infty$. Then there exists a monotone increasing function $h(R)$ with $h(R) / R \rightarrow 0$, as $R \rightarrow \infty$, such that

$$
\lim _{R \rightarrow \infty}\left(\frac{R}{h(R)}\right) \alpha(R)=0 .
$$


Proof. We define

$$
h(R)=\max _{r<R}(r \sqrt{\alpha(r)}) .
$$

Then $h(R)$ is monotone increasing and $h(R) / R \rightarrow 0$, as $R \rightarrow \infty$. Indeed, for any $\epsilon>0$

$$
h(R) \leq \max _{r<\epsilon R}(r \sqrt{\alpha(r)})+\max _{\epsilon R<r<R}(r \sqrt{\alpha(r)}) \leq \epsilon R \sqrt{\alpha(0)}+R \sqrt{\alpha(\epsilon R)} .
$$

Therefore,

$$
\varlimsup_{R \rightarrow \infty} \frac{h(R)}{R} \leq \epsilon \sqrt{\alpha(0)}
$$

Hence, $h(R) / R \rightarrow 0$, as $R \rightarrow \infty$. By definition of $h(R)$ we have $h(R) \geq R \sqrt{\alpha(R)}$. Therefore,

$$
\frac{R}{h(R)} \leq \frac{1}{\sqrt{\alpha(R)}}
$$

Thus,

$$
\left(\frac{R}{h(R)}\right) \alpha(R) \leq \sqrt{\alpha(R)} \rightarrow 0, \text { as } R \rightarrow \infty
$$

Now let us prove Lemma 3.7. First observe that for any $\phi \in \mathcal{S}_{0}$

$$
\lim _{R \rightarrow \infty} \int_{B_{R}}|\nabla \phi|^{2} d \boldsymbol{x}=\|\nabla \phi\|_{2}^{2}
$$

while

$$
\lim _{R \rightarrow \infty} \int_{A_{R}(h(R))}|\nabla \phi|^{2} d \boldsymbol{x}=0 .
$$

Hence, we only need to prove that there exists $c \in \mathbb{R}^{m}$ and a monotone increasing function $h(R)=o(R)$ such that

$$
\lim _{R \rightarrow \infty} \frac{1}{h(R)^{2}} \int_{A_{R}(h(R))}|\boldsymbol{\phi}-\boldsymbol{c}|^{2} d \boldsymbol{x}=0 .
$$

Remark 3.6 implies that we need to prove (B.2) for $d \geq 3$. In that case, the constant $c \in \mathbb{R}^{m}$ is chosen so that $\phi-c \in L^{\frac{2 d}{d-2}}\left(\mathbb{R}^{d} ; \mathbb{R}^{m}\right)$, which is possible by Theorem A.1. The Hölder inequality gives us

$$
\frac{1}{h(R)^{2}} \int_{A_{R}(h(R))}|\boldsymbol{\phi}-\boldsymbol{c}|^{2} d \boldsymbol{x} \leq C\left(\frac{R}{h(R)}\right)^{\frac{2(d-1)}{d}}\left(\int_{A_{R}(h(R))}|\boldsymbol{\phi}-\boldsymbol{c}|^{\frac{2 d}{d-2}} d \boldsymbol{x}\right)^{\frac{d-2}{d}} .
$$

By Theorem A.1

$$
\alpha(R)=\left(\int_{|\boldsymbol{x}| \geq R / 2}|\boldsymbol{\phi}-\boldsymbol{c}|^{\frac{2 d}{d-2}} d \boldsymbol{x}\right)^{\frac{d-2}{2(d-1)}} \rightarrow 0, \text { as } R \rightarrow \infty .
$$

We see that in each of the three cases we have a function $\alpha(R) \rightarrow 0$, as $R \rightarrow \infty$, which is independent of $h(R)$. The application of Lemma B.1 concludes the proof of Lemma 3.7. 


\section{Proof of Theorem 3.9}

Step 1: Asymptotics of $\int_{B_{R}}|\nabla \boldsymbol{\phi}|^{2} d \boldsymbol{x}$.

We write $\boldsymbol{x}=\boldsymbol{p}+\boldsymbol{R}^{T} \boldsymbol{t}$ and $|\boldsymbol{x}|^{2}=|\boldsymbol{p}|^{2}+|\boldsymbol{t}|^{2}$. Therefore,

$$
\begin{gathered}
B_{R} \subset V_{R}=\left\{\boldsymbol{x} \in \mathbb{R}^{d}: \boldsymbol{x}=\boldsymbol{p}+\boldsymbol{R}^{T} \boldsymbol{t},|\boldsymbol{t}| \leq R,|\boldsymbol{p}| \leq R\right\} . \\
\int_{V_{R}}|\nabla \boldsymbol{\phi}|^{2} d \boldsymbol{x}=\int_{\{|\boldsymbol{t}|<R\}} \int_{\{|\boldsymbol{p}|<R\}}\left\{\left|\boldsymbol{\psi}_{\boldsymbol{t}}\right|^{2}+\left|\boldsymbol{\psi}_{\boldsymbol{p}}\right|^{2}\right\} d \boldsymbol{t} d \boldsymbol{p} .
\end{gathered}
$$

If we make a change of variables $\boldsymbol{p}=R \boldsymbol{u}$ we obtain

$$
\frac{1}{R^{d-k}} \int_{V_{R}}|\nabla \boldsymbol{\phi}|^{2} d \boldsymbol{x}=\int_{\{|\boldsymbol{t}|<R\}} \int_{\{|\boldsymbol{u}|<1\}}\left\{\left|\boldsymbol{\psi}_{\boldsymbol{t}}(\boldsymbol{t}, R \boldsymbol{u})\right|^{2}+\left|\boldsymbol{\psi}_{\boldsymbol{p}}(\boldsymbol{t}, R \boldsymbol{u})\right|^{2}\right\} d \boldsymbol{u} d \boldsymbol{t} .
$$

Hence,

$$
\frac{1}{R^{d-k}} \int_{V_{R}}|\nabla \phi|^{2} d \boldsymbol{x} \leq \int_{\{|\boldsymbol{u}|<1\}} \int_{\mathbb{R}^{k}}\left\{\left|\boldsymbol{\psi}_{\boldsymbol{t}}(\boldsymbol{t}, R \boldsymbol{u})\right|^{2}+\left|\boldsymbol{\psi}_{\boldsymbol{p}}(\boldsymbol{t}, R \boldsymbol{u})\right|^{2}\right\} d \boldsymbol{t} d \boldsymbol{u} .
$$

By the Riemann-Lebesgue lemma we get

$$
\varlimsup_{R \rightarrow \infty} \frac{1}{R^{d-k}} \int_{V_{R}}|\nabla \boldsymbol{\phi}|^{2} d \boldsymbol{x} \leq \omega_{d-k} \quad f_{Q_{d-k}} \int_{\mathbb{R}^{k}}\left\{\left|\boldsymbol{\psi}_{\boldsymbol{t}}(\boldsymbol{t}, \boldsymbol{p})\right|^{2}+\left|\boldsymbol{\psi}_{\boldsymbol{p}}(\boldsymbol{t}, \boldsymbol{p})\right|^{2}\right\} d \boldsymbol{t} d \boldsymbol{p},
$$

where $\omega_{n}$ is the volume of the $n$-dimensional unit ball. Thus,

$$
\varlimsup_{R \rightarrow \infty} \frac{1}{R^{d-k}} \int_{B_{R}}|\nabla \phi|^{2} d \boldsymbol{x} \leq \omega_{d-k} \quad f_{Q_{d-k}} \int_{\mathbb{R}^{k}}\left\{\left|\boldsymbol{\psi}_{\boldsymbol{t}}(\boldsymbol{t}, \boldsymbol{p})\right|^{2}+\left|\boldsymbol{\psi}_{\boldsymbol{p}}(\boldsymbol{t}, \boldsymbol{p})\right|^{2}\right\} d \boldsymbol{t} d \boldsymbol{p} .
$$

To get the reverse inequality we write

$$
\int_{B_{R}}|\nabla \phi|^{2} d \boldsymbol{x}=\int_{\{|\boldsymbol{t}|<R\}} \int_{\{|\boldsymbol{p}|<r(R, t)\}}\left\{\left|\boldsymbol{\psi}_{\boldsymbol{t}}\right|^{2}+\left|\boldsymbol{\psi}_{\boldsymbol{p}}\right|^{2}\right\} d \boldsymbol{p} d \boldsymbol{t},
$$

where $r(R, \boldsymbol{t})=\sqrt{R^{2}-|\boldsymbol{t}|^{2}}$. If we make a change of variables $\boldsymbol{p}=r(R, \boldsymbol{t}) \boldsymbol{u}$ we obtain

$$
\int_{B_{R}}|\nabla \boldsymbol{\phi}|^{2} d \boldsymbol{x}=\int_{\{|\boldsymbol{t}|<R\}} r(R, \boldsymbol{t})^{d-k} \int_{\{|\boldsymbol{u}|<1\}}\left\{\left|\boldsymbol{\psi}_{\boldsymbol{t}}(\boldsymbol{t}, r(R, \boldsymbol{t}) \boldsymbol{u})\right|^{2}+\left|\boldsymbol{\psi}_{\boldsymbol{p}}(\boldsymbol{t}, r(R, \boldsymbol{t}) \boldsymbol{u})\right|^{2}\right\} d \boldsymbol{u} d \boldsymbol{t} .
$$

By the Riemann-Lebesgue lemma we get

$$
\lim _{R \rightarrow \infty} \int_{\{|\boldsymbol{u}|<1\}}\left\{\left|\boldsymbol{\psi}_{\boldsymbol{t}}(\boldsymbol{t}, r(R, \boldsymbol{t}) \boldsymbol{u})\right|^{2}+\left|\boldsymbol{\psi}_{\boldsymbol{p}}(\boldsymbol{t}, r(R, \boldsymbol{t}) \boldsymbol{u})\right|^{2}\right\} d \boldsymbol{u}=\omega_{d-k} \quad f_{Q_{d-k}}\left\{\left|\boldsymbol{\psi}_{\boldsymbol{t}}(\boldsymbol{t}, \boldsymbol{p})\right|^{2}+\left|\boldsymbol{\psi}_{\boldsymbol{p}}(\boldsymbol{t}, \boldsymbol{p})\right|^{2}\right\} d \boldsymbol{p}
$$

for a.e. $t \in \mathbb{R}^{k}$. By Fatous's lemma we get

$$
\varliminf_{R \rightarrow \infty} \frac{1}{R^{d-k}} \int_{B_{R}}|\nabla \phi|^{2} d \boldsymbol{x} \geq \omega_{d-k} \int_{\mathbb{R}^{k}} f_{Q_{d-k}}\left\{\left|\boldsymbol{\psi}_{\boldsymbol{t}}(\boldsymbol{t}, \boldsymbol{p})\right|^{2}+\left|\boldsymbol{\psi}_{\boldsymbol{p}}(\boldsymbol{t}, \boldsymbol{p})\right|^{2}\right\} d \boldsymbol{p} d \boldsymbol{t} .
$$

Hence, we obtain the asymptotics of $\int_{B_{R}}|\nabla \boldsymbol{\phi}|^{2} d \boldsymbol{x}$ :

$$
\lim _{R \rightarrow \infty} \frac{1}{R^{d-k}} \int_{B_{R}}|\nabla \phi|^{2} d \boldsymbol{x}=\omega_{d-k} \int_{\mathbb{R}^{k}} f_{Q_{d-k}}\left\{\left|\boldsymbol{\psi}_{\boldsymbol{t}}(\boldsymbol{t}, \boldsymbol{p})\right|^{2}+\left|\boldsymbol{\psi}_{\boldsymbol{p}}(\boldsymbol{t}, \boldsymbol{p})\right|^{2}\right\} d \boldsymbol{p} d \boldsymbol{t} .
$$

In particular, we get

$$
\varlimsup_{R \rightarrow \infty} f_{B_{R}}|\nabla \phi|^{2} d \boldsymbol{x} \leq \varlimsup_{R \rightarrow \infty} \frac{\omega_{d}}{R^{d}} \int_{V_{R}}|\nabla \phi|^{2} d \boldsymbol{x}=0
$$


establishing (3.19).

Step 2: Proof of (3.17).

For any $h(R)=o(R)$ we have, using (C.1)

$$
\frac{\int_{A_{R}(h(R))}|\nabla \boldsymbol{\phi}|^{2} d \boldsymbol{x}}{\int_{B_{R}}|\nabla \boldsymbol{\phi}|^{2} d \boldsymbol{x}}=\frac{\int_{B_{R}}|\nabla \boldsymbol{\phi}|^{2} d \boldsymbol{x}-\int_{B_{R-h(R)}}|\nabla \boldsymbol{\phi}|^{2} d \boldsymbol{x}}{\int_{B_{R}}|\nabla \boldsymbol{\phi}|^{2} d \boldsymbol{x}}=1-\left(1-\frac{h(R)}{R}\right)^{d-k} u_{R},
$$

where $u_{R} \rightarrow 1$, as $R \rightarrow \infty$. Thus, (3.17) is proved.

Step 3. Proof of (3.18). We have

$$
\int_{A_{R}(h(R))}|\boldsymbol{\phi}|^{2} d \boldsymbol{x} \leq \int_{|\boldsymbol{t}|<R} \int_{|\boldsymbol{p}|<R}|\boldsymbol{\psi}(\boldsymbol{t}, \boldsymbol{p})|^{2} d \boldsymbol{p} d \boldsymbol{t}
$$

The periodicity in $\boldsymbol{p}$ variable implies that for any domain $\Omega \subset \mathbb{R}^{d-k}$

$$
\int_{\Omega}|\boldsymbol{\psi}(\boldsymbol{t}, \boldsymbol{p})|^{2} d \boldsymbol{p} \leq v(\Omega) \quad f_{Q_{d-k}}|\boldsymbol{\psi}(\boldsymbol{t}, \boldsymbol{p})|^{2} d \boldsymbol{p},
$$

where $v(\Omega)$ is the $(d-k)$-volume of all period cells intersecting $\Omega$. We further estimate that

$$
v(\Omega) \leq\left|\Omega+B_{M}\right|
$$

where $M$ is the diameter of the period cell $Q_{d-k}$. When $R>M$ we obtain

$$
v(\{|\boldsymbol{p}| \leq R+M\}) \leq \omega_{d-k}(2 R)^{d-k}
$$

Hence, we get the estimate

$$
\varliminf_{R \rightarrow \infty} \frac{\frac{1}{h(R)^{2}} \int_{A_{R}(h(R))}|\boldsymbol{\phi}|^{2} d \boldsymbol{x}}{\int_{B_{R}}|\nabla \boldsymbol{\phi}|^{2} d \boldsymbol{x}} \leq \frac{2^{d-k}}{\|\nabla \boldsymbol{\phi}\|_{L^{2}(Y)}^{2}} \varliminf_{R \rightarrow \infty} \frac{1}{h(R)^{2}} \int_{|\boldsymbol{t}|<R} f_{Q_{d-k}}|\boldsymbol{\psi}(\boldsymbol{t}, \boldsymbol{p})|^{2} d \boldsymbol{p} d \boldsymbol{t} .
$$

For convenience we introduce the truncated $L^{2}$ norm

$$
\|\boldsymbol{f}\|_{2, R}^{2}=\int_{B_{R}} \int_{Q_{d-k}}|\boldsymbol{f}(\boldsymbol{t}, \boldsymbol{p})|^{2} d \boldsymbol{p} d \boldsymbol{t}
$$

Lemma C.1. For every $\boldsymbol{\psi} \in \mathcal{S}_{k}\left(Q_{d-k}\right)$ there exists a constant $\boldsymbol{c} \in \mathbb{R}^{m}$ such that

$$
\varliminf_{R \rightarrow \infty} \frac{\|\boldsymbol{\psi}-\boldsymbol{c}\|_{2, R}^{2}}{R^{2}}=0
$$

Proof. The proof of the Lemma is different depending on whether $k=1, k=2$ or $k \geq 3$.

If $k=1$ we can use the assumption of uniform boundedness of $\boldsymbol{\psi}$ and conclude that

$$
\frac{\|\boldsymbol{\psi}\|_{2, R}^{2}}{R^{2}} \leq \frac{2\|\boldsymbol{\psi}\|_{\infty}^{2}}{R} \rightarrow 0, \text { as } R \rightarrow \infty .
$$

If $k \geq 3$ then, according to Theorem A.1, for a.e. $\boldsymbol{p} \in Q_{d-k}$ there exists a unique vector $\boldsymbol{c}(\boldsymbol{p})$ such that

$$
\int_{\mathbb{R}^{k}}|\boldsymbol{\psi}(\boldsymbol{t}, \boldsymbol{p})-\boldsymbol{c}(\boldsymbol{p})|^{\frac{2 k}{k-2}}<\infty .
$$

However, we need a sharper statement 
Lemma C.2. There exists $\boldsymbol{c} \in \mathbb{R}^{m}$ such that $\boldsymbol{c}(\boldsymbol{p})=\boldsymbol{c}$ for a.e. $\boldsymbol{p} \in Q_{d-k}$.

Proof. Let

$$
\langle\boldsymbol{\psi}\rangle_{Q_{d-k}}(\boldsymbol{t})=\frac{1}{\left|Q_{d-k}\right|} \int_{Q_{d-k}} \boldsymbol{\psi}(\boldsymbol{t}, \boldsymbol{p}) d \boldsymbol{p}
$$

The Poincaré inequality implies

$$
\left\|\boldsymbol{\psi}-\langle\boldsymbol{\psi}\rangle_{Q_{d-k}}(\boldsymbol{t})\right\|_{2, R}^{2} \leq C\left\|\boldsymbol{\psi}_{\boldsymbol{p}}\right\|_{2, R}^{2} .
$$

Therefore, $\left\|\boldsymbol{\psi}-\langle\boldsymbol{\psi}\rangle_{Q_{d-k}}(\boldsymbol{t})\right\|_{2, R}$ is bounded as $R \rightarrow \infty$. Next observe that $\langle\boldsymbol{\psi}\rangle_{Q_{d-k}}(\boldsymbol{t}) \in \mathcal{S}$ as a function of $\boldsymbol{t}$. Hence, there exists $\boldsymbol{c} \in \mathbb{R}^{m}$ such that $\langle\boldsymbol{\psi}\rangle_{Q_{d-k}}(\boldsymbol{t})-\boldsymbol{c} \in L^{\frac{2 k}{k-2}}\left(\mathbb{R}^{k}\right)$. It follows that

$$
\|\boldsymbol{c}(\boldsymbol{p})-\boldsymbol{c}\|_{2, R} \leq\|\boldsymbol{c}(\boldsymbol{p})-\boldsymbol{\psi}\|_{2, R}+\left\|\boldsymbol{\psi}-\langle\boldsymbol{\psi}\rangle_{Q_{d-k}}(\boldsymbol{t})\right\|_{2, R}+\left\|\langle\boldsymbol{\psi}\rangle_{Q_{d-k}}(\boldsymbol{t})-\boldsymbol{c}\right\|_{2, R} .
$$

Let us apply the Hölder inequality to the first and third term on the left-hand side of the above inequality.

$$
\begin{gathered}
\|\boldsymbol{c}(\boldsymbol{p})-\boldsymbol{\psi}\|_{2, R}^{2} \leq C R^{2} \int_{Q_{d-k}}\left(\int_{\mathbb{R}^{k}}|\boldsymbol{c}(\boldsymbol{p})-\boldsymbol{\psi}|^{\frac{2 k}{k-2}} d \boldsymbol{t}\right)^{\frac{k-2}{k}} d \boldsymbol{p} . \\
\left\|\langle\boldsymbol{\psi}\rangle_{Q_{d-k}}(\boldsymbol{t})-\boldsymbol{c}\right\|_{2, R}^{2} \leq C R^{2}\left|Q_{d-k}\right|\left(\int_{\mathbb{R}^{k}}\left|\langle\boldsymbol{\psi}\rangle_{Q_{d-k}}(\boldsymbol{t})-\boldsymbol{c}\right|^{\frac{2 k}{k-2}} d \boldsymbol{t}\right)^{\frac{k-2}{k}} .
\end{gathered}
$$

We conclude that

$$
\varlimsup_{R \rightarrow \infty} \frac{1}{R^{2}}\|\boldsymbol{c}(\boldsymbol{p})-\boldsymbol{c}\|_{2, R}^{2}<+\infty
$$

However, this would contradict

$$
\|\boldsymbol{c}(\boldsymbol{p})-\boldsymbol{c}\|_{2, R}^{2}=\left|B_{R}\right| \int_{Q_{d-k}}|\boldsymbol{c}(\boldsymbol{p})-\boldsymbol{c}|^{2} d \boldsymbol{p}
$$

unless $\boldsymbol{c}(\boldsymbol{p})=\boldsymbol{c}$ for a.e. $\boldsymbol{p} \in Q_{d-k}$.

We will now establish Lemma C.1 in which the constant vector $\boldsymbol{c}$ is coming from Lemma C.2. For simplicity of notation $\boldsymbol{\psi}(\boldsymbol{t}, \boldsymbol{p})$ will now stand for $\boldsymbol{\psi}-\boldsymbol{c}$. In order to prove Lemma C.1 we split the $\boldsymbol{t}$-integral in the definition of $\|\boldsymbol{\psi}\|_{2, R}$ into the integral over the ball $\{|\boldsymbol{t}|<\epsilon R\}$ and the annulus $\{\epsilon R<|\boldsymbol{t}|<R\}$. Then we apply the same Hölder inequality to both integrals and obtain the estimate

$$
\begin{aligned}
\frac{1}{R^{2}} \int_{|\boldsymbol{t}|<R}|\boldsymbol{\psi}(\boldsymbol{t}, \boldsymbol{p})|^{2} d \boldsymbol{t} \leq \omega_{k}^{\frac{k}{2}} \epsilon^{2}\left(\int_{|\boldsymbol{t}|<\epsilon R}|\boldsymbol{\psi}(\boldsymbol{t}, \boldsymbol{p})|^{\frac{2 k}{k-2}} d \boldsymbol{t}\right)^{\frac{k-2}{k}} & \\
& +\omega_{k}^{\frac{k}{2}}\left(1-\epsilon^{k}\right)^{\frac{k}{2}}\left(\int_{\epsilon R<|\boldsymbol{t}|<R}|\boldsymbol{\psi}(\boldsymbol{t}, \boldsymbol{p})|^{\frac{2 k}{k-2}} d \boldsymbol{t}\right)^{\frac{k-2}{k}} .
\end{aligned}
$$

Lemma C.2 then implies that for a.e. $\boldsymbol{p} \in Q_{d-k}$

$$
\varlimsup_{R \rightarrow \infty} \frac{1}{R^{2}} \int_{|\boldsymbol{t}|<R}|\boldsymbol{\psi}(\boldsymbol{t}, \boldsymbol{p})|^{2} d \boldsymbol{t} \leq \omega_{k}^{\frac{k}{2}} \epsilon^{2}\left(\int_{\mathbb{R}^{k}}|\boldsymbol{\psi}(\boldsymbol{t}, \boldsymbol{p})|^{\frac{2 k}{k-2}} d \boldsymbol{t}\right)^{\frac{k-2}{k}} .
$$

Thus, for a.e. $\boldsymbol{p} \in Q_{d-k}$

$$
\lim _{R \rightarrow \infty} \frac{1}{R^{2}} \int_{|\boldsymbol{t}|<R}|\boldsymbol{\psi}(\boldsymbol{t}, \boldsymbol{p})|^{2} d \boldsymbol{t}=0
$$


By Hölder inequality and Theorem A.1

$$
\frac{1}{R^{2}} \int_{|\boldsymbol{t}|<R}|\boldsymbol{\psi}(\boldsymbol{t}, \boldsymbol{p})|^{2} d \boldsymbol{t} \leq \omega_{k}^{\frac{2}{k}}\left(\int_{|\boldsymbol{t}|<R}|\boldsymbol{\psi}(\boldsymbol{t}, \boldsymbol{p})|^{\frac{2 k}{k-2}} d \boldsymbol{t}\right)^{\frac{k-2}{k}} \leq C \int_{\mathbb{R}^{k}}\left|\boldsymbol{\psi}_{\boldsymbol{t}}(\boldsymbol{t}, \boldsymbol{p})\right|^{2} d \boldsymbol{t} .
$$

By the Lebesgue dominated convergence theorem $\|\boldsymbol{\psi}\|_{2, R}^{2} / R^{2} \rightarrow 0$, as $R \rightarrow \infty$, since the function

$$
\Phi(\boldsymbol{p})=\int_{\mathbb{R}^{k}}\left|\boldsymbol{\psi}_{\boldsymbol{t}}(\boldsymbol{t}, \boldsymbol{p})\right|^{2} d \boldsymbol{t}
$$

is integrable over $Q_{d-k}$.

The case $k=2$ is the most delicate. Let us define

$$
\boldsymbol{c}_{R}(\boldsymbol{p})=f_{|\boldsymbol{t}|<R} \psi(\boldsymbol{t}, \boldsymbol{p}) d \boldsymbol{t} .
$$

Let $R_{n} \rightarrow \infty$ be a strictly monotone sequence such that

$$
\lim _{n \rightarrow \infty}\left\langle\boldsymbol{c}_{R_{n}}\right\rangle_{Q_{d-2}}=\boldsymbol{c}
$$

for some vector $c \in \mathbb{R}^{m}$. We claim that

$$
\lim _{R \rightarrow \infty}\left\|\boldsymbol{c}_{R}(\boldsymbol{p})-\left\langle\boldsymbol{c}_{R}\right\rangle_{Q_{d-2}}\right\|_{2} d \boldsymbol{p}=0 .
$$

Indeed,

$$
\left\|\boldsymbol{c}_{R}(\boldsymbol{p})-\left\langle\boldsymbol{c}_{R}\right\rangle_{Q_{d-2}}\right\|_{2}^{2} \leq C f_{|\boldsymbol{t}|<R} \int_{Q_{d-2}}\left|\boldsymbol{\psi}(\boldsymbol{t}, \boldsymbol{p})-\langle\boldsymbol{\psi}\rangle_{Q_{d-2}}(\boldsymbol{t})\right|^{2} d \boldsymbol{p} d \boldsymbol{t} .
$$

Applying the Poincaré inequality for the inner integral we get

$$
\left\|\boldsymbol{c}_{R}-\left\langle\boldsymbol{c}_{R}\right\rangle_{Q_{d-2}}\right\|_{2}^{2} \leq C \quad f_{|\boldsymbol{t}|<R} \int_{Q_{d-2}}\left|\boldsymbol{\psi}_{\boldsymbol{p}}(\boldsymbol{t}, \boldsymbol{p})\right|^{2} d \boldsymbol{p} d \boldsymbol{t} \rightarrow 0, \text { as } R \rightarrow \infty .
$$

We now prove that

$$
\lim _{n \rightarrow \infty} \frac{1}{R^{2}}\|\boldsymbol{\psi}(\boldsymbol{t}, \boldsymbol{p})-\boldsymbol{c}\|_{2, R}^{2}=0
$$

By triangle inequality we have

$$
\|\boldsymbol{\psi}(\boldsymbol{t}, \boldsymbol{p})-\boldsymbol{c}\|_{2, R} \leq\left\|\boldsymbol{\psi}-\boldsymbol{c}_{R}(\boldsymbol{p})\right\|_{2, R}+\left\|\boldsymbol{c}_{R}(\boldsymbol{p})-\left\langle\boldsymbol{c}_{R}\right\rangle_{Q_{d-2}}\right\|_{2, R}+\left\|\left\langle\boldsymbol{c}_{R}\right\rangle_{Q_{d-2}}-\boldsymbol{c}\right\|_{2, R} .
$$

We compute

$$
\left\|\left\langle\boldsymbol{c}_{R}\right\rangle_{Q_{d-2}}-\boldsymbol{c}\right\|_{2, R}^{2}=\left|Q_{d-2}\right| \pi R^{2}\left|\left\langle\boldsymbol{c}_{R}\right\rangle_{Q_{d-2}}-\boldsymbol{c}\right|^{2} .
$$

Hence,

$$
\begin{gathered}
\lim _{n \rightarrow \infty} \frac{1}{R_{n}^{2}}\left\|\left\langle\boldsymbol{c}_{R_{n}}\right\rangle_{Q_{d-2}}-\boldsymbol{c}\right\|_{2, R}^{2}=0 . \\
\left\|\boldsymbol{c}_{R}(\boldsymbol{p})-\left\langle\boldsymbol{c}_{R}\right\rangle_{Q_{d-2}}\right\|_{2, R}^{2}=\pi R^{2}\left\|\boldsymbol{c}_{R}-\left\langle\boldsymbol{c}_{R}\right\rangle_{Q_{d-2}}\right\|_{2}^{2} .
\end{gathered}
$$

Therefore,

$$
\lim _{R \rightarrow \infty} \frac{1}{R^{2}}\left\|\boldsymbol{c}_{R}(\boldsymbol{p})-\left\langle\boldsymbol{c}_{R}\right\rangle_{Q_{d-2}}\right\|_{2, R}^{2}=0
$$

Finally, we have

$$
\left\|\boldsymbol{\psi}-\boldsymbol{c}_{R}(\boldsymbol{p})\right\|_{2, R}^{2}=\int_{Q_{d-2}} \int_{\{|\boldsymbol{t}|<\epsilon R\}}\left|\boldsymbol{\psi}-\boldsymbol{c}_{R}(\boldsymbol{p})\right|^{2} d \boldsymbol{t} d \boldsymbol{p}+\int_{Q_{d-2}} \int_{\{\epsilon R<|\boldsymbol{t}|<R\}}\left|\boldsymbol{\psi}-\boldsymbol{c}_{R}(\boldsymbol{p})\right|^{2} d \boldsymbol{t} d \boldsymbol{p} .
$$


Using the uniform boundedness of $\boldsymbol{\psi}$ for the first term and the Poincaré inequality for the second term we get

$$
\left\|\boldsymbol{\psi}-\boldsymbol{c}_{R}(\boldsymbol{p})\right\|_{2, R}^{2} \leq C \epsilon^{2} R^{2}\|\boldsymbol{\psi}-\boldsymbol{c}\|_{\infty}^{2}+R^{2} C_{\epsilon} \int_{Q_{d-2}} \int_{\{\epsilon R<|\boldsymbol{t}|<R\}}\left|\boldsymbol{\psi}_{\boldsymbol{t}}(\boldsymbol{t}, \boldsymbol{p})\right|^{2} d \boldsymbol{t} \boldsymbol{p} .
$$

Thus,

$$
\varlimsup_{R \rightarrow \infty} \frac{1}{R^{2}}\left\|\boldsymbol{\psi}-\boldsymbol{c}_{R}(\boldsymbol{p})\right\|_{2, R}^{2} \leq C \epsilon^{2}\|\boldsymbol{\psi}-\boldsymbol{c}\|_{\infty}^{2}
$$

The arbitrariness of $\epsilon>0$ implies that

$$
\lim _{R \rightarrow \infty} \frac{1}{R^{2}}\left\|\boldsymbol{\psi}-\boldsymbol{c}_{R}(\boldsymbol{p})\right\|_{2, R}^{2}=0
$$

Lemma C.1 is proved now.

Let $R_{n} \rightarrow \infty$ be the monotone increasing sequence for which $\|\boldsymbol{\psi}-\boldsymbol{c}\|_{2, R_{n}} / R_{n} \rightarrow 0$, as $n \rightarrow \infty$. Let

$$
\alpha(R)=\frac{\|\boldsymbol{\psi}-\boldsymbol{c}\|_{2, R_{n}}}{R_{n}}, \quad R_{n} \leq R<R_{n+1} .
$$

Then $\alpha(R) \rightarrow 0$, as $R \rightarrow \infty$. By Lemma B.1 there exists a monotone increasing function $h(R)$ such that $h(R) / R \rightarrow 0$, as $R \rightarrow \infty$ and

$$
\lim _{R \rightarrow \infty}\left(\frac{R}{h(R)}\right) \alpha(R)=0
$$

Hence,

$$
\varliminf_{R \rightarrow \infty}\left(\frac{\|\boldsymbol{\psi}-\boldsymbol{c}\|_{2, R}}{h(R)}\right)^{2} \leq \lim _{n \rightarrow \infty}\left(\frac{R_{n}}{h\left(R_{n}\right)} \alpha\left(R_{n}\right)\right)^{2}=0 .
$$

The estimate (C.2) together with (3.17) now implies (3.18). Thus, we have proved that $\mathcal{C}_{k} \subset \mathcal{S}_{*}$ for any $1 \leq k \leq d$.

Step 3: Proof of the formula (3.24). We have

$$
\frac{1}{R^{d-k}} \int_{B_{R}} W^{\circ}(\boldsymbol{F}, \nabla \phi) d \boldsymbol{x}=\int_{|\boldsymbol{u}| \leq 1} \int_{|\boldsymbol{t}| \leq R \sqrt{1-|\boldsymbol{u}|^{2}}} W^{\circ}\left(\boldsymbol{F}, \boldsymbol{\psi}_{\boldsymbol{t}}(\boldsymbol{t}, R \boldsymbol{u}) \boldsymbol{R}+\boldsymbol{\psi}_{\boldsymbol{p}}(\boldsymbol{t}, R \boldsymbol{u}) \boldsymbol{Q}\right) d \boldsymbol{t} d \boldsymbol{u}
$$

By the Riemann-Lebesgue lemma

$$
\begin{aligned}
\lim _{R \rightarrow \infty} \int_{|\boldsymbol{u}| \leq 1} \int_{\mathbb{R}^{k}} W^{\circ}\left(\boldsymbol{F}, \boldsymbol{\psi}_{\boldsymbol{t}}(\boldsymbol{t}, R \boldsymbol{u}) \boldsymbol{R}+\boldsymbol{\psi}_{\boldsymbol{p}}(\boldsymbol{t}, R \boldsymbol{u}) \boldsymbol{Q}\right) d \boldsymbol{t} d \boldsymbol{u} & = \\
\omega_{d-k} & f_{Q_{d-k}} \int_{\mathbb{R}^{k}} W^{\circ}\left(\boldsymbol{F}, \boldsymbol{\psi}_{\boldsymbol{t}}(\boldsymbol{t}, \boldsymbol{p}) \boldsymbol{R}+\boldsymbol{\psi}_{\boldsymbol{p}}(\boldsymbol{t}, \boldsymbol{p}) \boldsymbol{Q}\right) d \boldsymbol{t} d \boldsymbol{p} .
\end{aligned}
$$

Thus, in order to finish the proof of the theorem we need to show that

$$
\rho=\lim _{R \rightarrow \infty} \int_{|\boldsymbol{u}| \leq 1} \int_{|\boldsymbol{t}| \geq R \sqrt{1-|\boldsymbol{u}|^{2}}} W^{\circ}\left(\boldsymbol{F}, \boldsymbol{\psi}_{\boldsymbol{t}}(\boldsymbol{t}, R \boldsymbol{u}) \boldsymbol{R}+\boldsymbol{\psi}_{\boldsymbol{p}}(\boldsymbol{t}, R \boldsymbol{u}) \boldsymbol{Q}\right) d \boldsymbol{t} d \boldsymbol{u}=0 .
$$

Recall that $\phi \in W^{1, \infty}\left(\mathbb{R}^{d} ; \mathbb{R}^{m}\right)$. Hence, there exists a number $C>0$ depending on $\|\phi\|_{1, \infty}$, but independent of $R$ such that

$$
\rho \leq C \varlimsup_{R \rightarrow \infty} \int_{|\boldsymbol{u}| \leq 1} \int_{|\boldsymbol{t}| \geq R \sqrt{1-|\boldsymbol{u}|^{2}}}\left\{\left|\boldsymbol{\psi}_{\boldsymbol{t}}(\boldsymbol{t}, R \boldsymbol{u})\right|^{2}+\left|\boldsymbol{\psi}_{\boldsymbol{p}}(\boldsymbol{t}, R \boldsymbol{u})\right|^{2}\right\} d \boldsymbol{t} d \boldsymbol{u} .
$$

For any $\epsilon \in(0,1)$ we have

$$
\int_{|\boldsymbol{u}| \leq 1} \int_{|\boldsymbol{t}| \geq R \sqrt{1-|\boldsymbol{u}|^{2}}}\left\{\left|\boldsymbol{\psi}_{\boldsymbol{t}}(\boldsymbol{t}, R \boldsymbol{u})\right|^{2}+\left|\boldsymbol{\psi}_{\boldsymbol{p}}(\boldsymbol{t}, R \boldsymbol{u})\right|^{2}\right\} d \boldsymbol{t} d \boldsymbol{u}=T_{1}(R, \epsilon)+T_{2}(R, \epsilon),
$$


where

$$
\begin{gathered}
T_{1}(R, \epsilon)=\int_{|\boldsymbol{u}| \leq 1-\epsilon} \int_{|\boldsymbol{t}| \geq R \sqrt{1-|\boldsymbol{u}|^{2}}}\left\{\left|\boldsymbol{\psi}_{\boldsymbol{t}}(\boldsymbol{t}, R \boldsymbol{u})\right|^{2}+\left|\boldsymbol{\psi}_{\boldsymbol{p}}(\boldsymbol{t}, R \boldsymbol{u})\right|^{2}\right\} d \boldsymbol{t} d \boldsymbol{u} \\
T_{2}(R, \epsilon)=\int_{1-\epsilon<|\boldsymbol{u}| \leq 1} \int_{|\boldsymbol{t}| \geq R \sqrt{1-|\boldsymbol{u}|^{2}}}\left\{\left|\boldsymbol{\psi}_{\boldsymbol{t}}(\boldsymbol{t}, R \boldsymbol{u})\right|^{2}+\left|\boldsymbol{\psi}_{\boldsymbol{p}}(\boldsymbol{t}, R \boldsymbol{u})\right|^{2}\right\} d \boldsymbol{t} d \boldsymbol{u} .
\end{gathered}
$$

If $|\boldsymbol{u}| \leq 1-\epsilon$ and $|\boldsymbol{t}| \geq R \sqrt{1-|\boldsymbol{u}|^{2}}$ then $|\boldsymbol{t}| \geq R \sqrt{\epsilon(2-\epsilon)}$. In particular, $|\boldsymbol{t}| \geq \sqrt{(2-\epsilon) / \epsilon}$, if $R>1 / \epsilon$. Therefore, by the Riemann-Lebesgue lemma

$$
\varlimsup_{R \rightarrow \infty} T_{1}(R, \epsilon) \leq T_{1}^{\infty}(\epsilon)=\omega_{d-k} \quad f_{Q_{d-k}} \int_{|\boldsymbol{t}| \geq \sqrt{(2-\epsilon) / \epsilon}}\left\{\left|\boldsymbol{\psi}_{\boldsymbol{t}}(\boldsymbol{t}, \boldsymbol{p})\right|^{2}+\left|\boldsymbol{\psi}_{\boldsymbol{p}}(\boldsymbol{t}, \boldsymbol{p})\right|^{2}\right\} d \boldsymbol{p} d \boldsymbol{t} .
$$

Also, by the Riemann-Lebesgue lemma

$$
\varlimsup_{R \rightarrow \infty} T_{2}(R, \epsilon) \leq T_{2}^{\infty}(\epsilon)=|\{1-\epsilon<|\boldsymbol{u}| \leq 1\}| \quad f_{Q_{d-k}} \int_{\mathbb{R}^{k}}\left\{\left|\boldsymbol{\psi}_{\boldsymbol{t}}(\boldsymbol{t}, \boldsymbol{p})\right|^{2}+\left|\boldsymbol{\psi}_{\boldsymbol{p}}(\boldsymbol{t}, \boldsymbol{p})\right|^{2}\right\} d \boldsymbol{p} d \boldsymbol{t} .
$$

We conclude that $\rho=0$, since

$$
\lim _{\epsilon \rightarrow 0} T_{1}^{\infty}(\epsilon)=\lim _{\epsilon \rightarrow 0} T_{2}^{\infty}(\epsilon)=0
$$

\section{Proof of Theorem 3.14}

We may assume, without loss of generality, that $\boldsymbol{n}=\boldsymbol{e}_{1}$. By Lemma 3.2 in [89], applied to the bounded domain $Q=[0,1]^{d}$, there exists a sequence of functions $\boldsymbol{u}_{n}(\boldsymbol{x})$ converging uniformly in $Q$ to $\boldsymbol{u}_{0}(\boldsymbol{x})=x_{1} \boldsymbol{a}$ and such that $\left\|\nabla \boldsymbol{u}_{n}\right\|_{\infty}$ is a bounded sequence and for all $1 \leq j \leq r$

$$
\lim _{n \rightarrow \infty}\left|\left\{\boldsymbol{x} \in Q: \operatorname{dist}\left(\nabla \boldsymbol{u}_{n}(\boldsymbol{x}), \boldsymbol{H}_{j}\right)<1 / n\right\}\right|=\lambda_{j} .
$$

Let $\boldsymbol{p}_{n}(\boldsymbol{x})$ denote the function defined in the layer $0<x_{1}<1$, which is periodic with periods $\boldsymbol{e}_{2}, \ldots, \boldsymbol{e}_{d}$ and equal to $\boldsymbol{u}_{n}(\boldsymbol{x})$ on $Q$. Finally, we let

$$
\boldsymbol{v}_{n}(\boldsymbol{x})= \begin{cases}\boldsymbol{a}, & \text { if } x_{1} \geq 1, \\ \mathbf{0}, & \text { if } x_{1} \leq 0, \\ \boldsymbol{p}_{n}(\boldsymbol{x}), & \text { if } 0<x_{1}<1,\end{cases}
$$

Clearly, $\boldsymbol{v}_{n}(\boldsymbol{x}) \rightarrow \boldsymbol{\phi}_{0}(\boldsymbol{x})$ uniformly in $\mathbb{R}^{d}$. However, the functions $\boldsymbol{v}_{n}(\boldsymbol{x})$ have jump discontinuities across the surfaces $\Gamma_{j, k}=\left\{x_{j}=k, 0<x_{1}<1\right\}, j=2, \ldots, d, k \in \mathbb{Z}$, as well as the planes $\Pi_{0}=\left\{x_{1}=0\right\}$ and $\Pi_{1}=\left\{x_{1}=1\right\}$. Let $\epsilon_{n}=\left\|\boldsymbol{v}_{n}-\phi_{0}\right\|_{\infty}$. Then $\epsilon_{n} \rightarrow 0$ as $n \rightarrow \infty$. Let

$$
\Gamma=\Pi_{0} \cup \Pi_{1} \cup\left(\bigcup_{j=1}^{d}\left(\bigcup_{k \in \mathbb{Z}} \Gamma_{j, k}\right)\right)
$$

be the entire singular set. When $n$ is sufficiently large there is a $C^{\infty}\left(\mathbb{R}^{d}\right)$ function $\eta_{n}(\boldsymbol{x})$ that is periodic with periods $\boldsymbol{e}_{2}, \ldots, \boldsymbol{e}_{d}$, which is equal to 0 on $\Gamma$ and 1 on $\left\{\boldsymbol{x} \in \mathbb{R}^{d}: \operatorname{dist}(\boldsymbol{x}, \Gamma)>\sqrt{\epsilon_{n}}\right\}$, and such that $\left\|\nabla \eta_{n}\right\|_{\infty} \leq C / \sqrt{\epsilon_{n}}$. It follows that the function

$$
\boldsymbol{\phi}_{n}(\boldsymbol{x})=\left(1-\eta_{n}(\boldsymbol{x})\right) \boldsymbol{\phi}_{0}(\boldsymbol{x})+\eta_{n}(\boldsymbol{x}) \boldsymbol{v}_{n}(\boldsymbol{x})
$$

is Lipschitz continuous with

$$
\left\|\nabla \phi_{n}\right\|_{\infty} \leq\left\|\nabla \phi_{0}\right\|_{\infty}+\left\|\nabla \boldsymbol{u}_{n}\right\|_{\infty}+C \sqrt{\epsilon_{n}} .
$$


Obviously, $\phi_{n}(\boldsymbol{x})$ converges uniformly to $\phi_{0}(\boldsymbol{x})$. In addition $\nabla \boldsymbol{\phi}_{n}(\boldsymbol{x})=0$ whenever $x_{1}<-\sqrt{\epsilon_{n}}$ or $x_{1}>$ $1+\sqrt{\epsilon_{n}}$. It follows that (3.19) holds. Observe that $\boldsymbol{\phi}_{n}(\boldsymbol{x})$ has periods $\boldsymbol{e}_{2}, \ldots, \boldsymbol{e}_{d}$, since both $\boldsymbol{v}_{n}(\boldsymbol{x})$ and $\eta_{n}(\boldsymbol{x})$ do. Thus, $\boldsymbol{\psi}_{n} \in \mathcal{S}_{1}^{0}$, where

$$
\boldsymbol{\psi}_{n}(t, \boldsymbol{p})=\phi_{n}\left(t \boldsymbol{e}_{1}+\sum_{j=2}^{d} p_{j} \boldsymbol{e}_{j}\right)
$$

Hence, $\phi_{n} \in \mathcal{C}_{1}$. To finish the proof of the theorem we need to establish (3.29). This is a consequence of the formula (3.24) and the relation

$$
\lim _{n \rightarrow \infty} \int_{\mathbb{R}}\left(\int_{[0,1]^{d-1}} W^{\circ}\left(\boldsymbol{F}, \nabla \phi_{n}\right) d x_{2} \ldots d x_{d}\right) d x_{1}=\lim _{n \rightarrow \infty} \int_{Q} W^{\circ}\left(\boldsymbol{F}, \nabla \boldsymbol{u}_{n}\right) d \boldsymbol{x}=J(\boldsymbol{F}, \nu) .
$$

\section{E Proof of Lemma 4.4}

The lemma is best proved in the $(\boldsymbol{t}, \boldsymbol{p})$ variables, where instead of $[0,1]^{d-k}$ periodic field $\boldsymbol{\psi}$ we use $Q_{d-k}$ periodic field, that we denote $\boldsymbol{\psi}$ as well, so that

$$
\phi(\boldsymbol{x})=\boldsymbol{\psi}(\boldsymbol{R} \boldsymbol{x}, \boldsymbol{Q x}) \quad \boldsymbol{\psi}(\boldsymbol{t}, \boldsymbol{p})=\phi\left(\boldsymbol{R}^{T} \boldsymbol{t}+\boldsymbol{Q}^{T} \boldsymbol{p}\right) .
$$

In terms of $\boldsymbol{\psi}$ equations (4.12) become

$$
\left\{\begin{array}{l}
\nabla_{\boldsymbol{t}} \cdot\left(\boldsymbol{P}\left(\boldsymbol{F}+\boldsymbol{\psi}_{\boldsymbol{t}} \boldsymbol{R}+\boldsymbol{\psi}_{\boldsymbol{p}} \boldsymbol{Q}\right) \boldsymbol{R}^{T}\right)+\nabla_{\boldsymbol{p}} \cdot\left(\boldsymbol{P}\left(\boldsymbol{F}+\boldsymbol{\psi}_{\boldsymbol{t}} \boldsymbol{R}+\boldsymbol{\psi}_{\boldsymbol{p}} \boldsymbol{Q}\right) \boldsymbol{Q}^{T}\right)=\mathbf{0} \\
\nabla_{\boldsymbol{t}} \cdot\left(\boldsymbol{R} \boldsymbol{P}^{*}\left(\boldsymbol{F}+\boldsymbol{\psi}_{\boldsymbol{t}} \boldsymbol{R}+\boldsymbol{\psi}_{\boldsymbol{p}} \boldsymbol{Q}\right) \boldsymbol{R}^{T}\right)=\nabla_{\boldsymbol{p}} \cdot\left(\boldsymbol{\psi}_{\boldsymbol{t}}^{T} \boldsymbol{P}\left(\boldsymbol{F}+\boldsymbol{\psi}_{\boldsymbol{t}} \boldsymbol{R}+\boldsymbol{\psi}_{\boldsymbol{p}} \boldsymbol{Q}\right) \boldsymbol{Q}^{T}\right) \\
\nabla_{\boldsymbol{p}} \cdot\left(\boldsymbol{Q} \boldsymbol{P}^{*}\left(\boldsymbol{F}+\boldsymbol{\psi}_{\boldsymbol{t}} \boldsymbol{R}+\boldsymbol{\psi}_{\boldsymbol{p}} \boldsymbol{Q}\right) \boldsymbol{Q}^{T}\right)=\nabla_{\boldsymbol{t}} \cdot\left(\boldsymbol{\psi}_{\boldsymbol{p}}^{T} \boldsymbol{P}\left(\boldsymbol{F}+\boldsymbol{\psi}_{\boldsymbol{t}} \boldsymbol{R}+\boldsymbol{\psi}_{\boldsymbol{p}} \boldsymbol{Q}\right) \boldsymbol{R}^{T}\right)
\end{array}\right.
$$

while relation (4.18) reads

$$
\left\{\begin{array}{l}
\int_{\mathbb{R}^{k}} \int_{Q_{d-k}} \boldsymbol{R} \widehat{\boldsymbol{P}}^{*} \boldsymbol{R}^{T} d \boldsymbol{p} d \boldsymbol{t}=\mathbf{0}, \\
\int_{\mathbb{R}^{k}} \int_{Q_{d-k}} \boldsymbol{\psi}_{\boldsymbol{p}}^{T} \widehat{\boldsymbol{P}} \boldsymbol{R}^{T} d \boldsymbol{p} d \boldsymbol{t}=\mathbf{0},
\end{array}\right.
$$

where

$$
\widehat{\boldsymbol{P}}=\widehat{\boldsymbol{P}}\left(\psi_{t} \boldsymbol{R}+\psi_{p} Q\right)=\boldsymbol{P}\left(\boldsymbol{F}+\psi_{t} \boldsymbol{R}+\psi_{p} Q\right)-\boldsymbol{P}(\boldsymbol{F}) .
$$

Replacing $\widehat{\boldsymbol{P}}^{*}$ by its expression from (4.15) and using equations (E.1) we obtain

$$
\nabla_{\boldsymbol{t}} \cdot\left(\boldsymbol{R} \widehat{\boldsymbol{P}}^{*} \boldsymbol{R}^{T}\right)=\nabla_{\boldsymbol{p}} \cdot\left(\boldsymbol{\psi}_{\boldsymbol{t}}^{T} \widehat{\boldsymbol{P}} \boldsymbol{Q}^{T}\right)-\nabla_{\boldsymbol{p}} \cdot\left(\boldsymbol{R} \boldsymbol{F}^{T} \boldsymbol{P}\left(\boldsymbol{F}+\boldsymbol{\psi}_{\boldsymbol{t}} \boldsymbol{R}+\boldsymbol{\psi}_{\boldsymbol{p}} \boldsymbol{Q}\right) \boldsymbol{Q}^{T}\right),
$$

since

$$
\nabla_{\boldsymbol{t}} \cdot\left(\boldsymbol{R} \boldsymbol{N}\left(\boldsymbol{\psi}_{\boldsymbol{t}} \boldsymbol{R}+\boldsymbol{\psi}_{\boldsymbol{p}} \boldsymbol{Q}\right) \boldsymbol{R}^{T}\right)=-\nabla_{\boldsymbol{t}} \cdot\left(\boldsymbol{\psi}_{\boldsymbol{t}}^{T} \boldsymbol{P}(\boldsymbol{F})\right)
$$

Let

$$
\begin{gathered}
\boldsymbol{f}_{1}(\boldsymbol{t})=\int_{Q_{d-k}} \boldsymbol{R} \widehat{\boldsymbol{P}}^{*} \boldsymbol{R}^{T} d \boldsymbol{p}, \\
\boldsymbol{f}_{2}(\boldsymbol{t})=\int_{Q_{d-k}} \boldsymbol{\psi}_{\boldsymbol{p}}^{T} \widehat{\boldsymbol{P}} \boldsymbol{R}^{T} d \boldsymbol{p}=\int_{Q_{d-k}} \boldsymbol{\psi}_{\boldsymbol{p}}^{T} \boldsymbol{P}\left(\boldsymbol{F}+\boldsymbol{\psi}_{\boldsymbol{t}} \boldsymbol{R}+\boldsymbol{\psi}_{\boldsymbol{p}} \boldsymbol{Q}\right) \boldsymbol{R}^{T} d \boldsymbol{p} .
\end{gathered}
$$

Integrating (E.3) over $Q_{d-k}$ and using the periodicity we obtain that $\nabla_{\boldsymbol{t}} \cdot \boldsymbol{f}_{1}(\boldsymbol{t})=\mathbf{0}$. Similarly, Integrating the third equation in (E.1) over $Q_{d-k}$ we conclude that $\nabla_{\boldsymbol{t}} \cdot \boldsymbol{f}_{2}(\boldsymbol{t})=\mathbf{0}$. We estimate

$$
\left|\widehat{\boldsymbol{P}}^{*}\right| \leq C\left(\left|\boldsymbol{\psi}_{\boldsymbol{t}}\right|^{2}+\left|\boldsymbol{\psi}_{\boldsymbol{p}}\right|^{2}\right), \quad\left|\boldsymbol{\psi}_{\boldsymbol{p}}^{T} \widehat{\boldsymbol{P}}\right| \leq C\left(\left|\boldsymbol{\psi}_{\boldsymbol{t}}\right|^{2}+\left|\boldsymbol{\psi}_{\boldsymbol{p}}\right|^{2}\right),
$$


since $\boldsymbol{\psi}_{\boldsymbol{t}}$ and $\boldsymbol{\psi}_{\boldsymbol{p}}$ are assumed to be uniformly bounded. Then $\boldsymbol{\phi} \in \mathcal{C}_{k}$ implies that $\left\{\boldsymbol{f}_{1}, \boldsymbol{f}_{2}\right\} \subset L^{1}\left(\mathbb{R}^{k}\right)$. The statement of the lemma follows from

$$
\int_{\mathbb{R}^{k}} \boldsymbol{f}_{1}(\boldsymbol{t}) d \boldsymbol{t}=\mathbf{0}, \quad \int_{\mathbb{R}^{k}} \boldsymbol{f}_{2}(\boldsymbol{t}) d \boldsymbol{t}=\mathbf{0},
$$

which is a consequence of a simple observation that any $L^{1}$ divergence-free vector field $\boldsymbol{f}(\boldsymbol{t})$ on $\mathbb{R}^{k}$ must satisfy $\int_{\mathbb{R}^{k}} \boldsymbol{f} d \boldsymbol{t}=\mathbf{0}$. Indeed, $\boldsymbol{f} \in L^{1}$ implies that its Fourier transform $\widehat{\boldsymbol{f}}(\boldsymbol{\omega})$ is continuous. $\nabla \cdot \boldsymbol{f}=0$ implies that $\boldsymbol{\omega} \cdot \widehat{\boldsymbol{f}}(\boldsymbol{\omega})=0$ for any $\boldsymbol{\omega} \in \mathbb{R}^{k}$. Fixing $\boldsymbol{\omega} \neq \mathbf{0}$ we obtain

$$
\frac{\boldsymbol{\omega} \cdot \widehat{\boldsymbol{f}}(\epsilon \boldsymbol{\omega})}{|\boldsymbol{\omega}|}=\frac{\epsilon \boldsymbol{\omega} \cdot \widehat{\boldsymbol{f}}(\epsilon \boldsymbol{\omega})}{|\epsilon \boldsymbol{\omega}|}=0 .
$$

Passing to the limit as $\epsilon \rightarrow 0$ and using continuity of $\widehat{\boldsymbol{f}}(\boldsymbol{\omega})$ we obtain $\boldsymbol{\omega} \cdot \widehat{\boldsymbol{f}}(\mathbf{0})=0$. Thus $\widehat{\boldsymbol{f}}(\mathbf{0})=\mathbf{0}$, since $\boldsymbol{\omega} \in \mathbb{R}^{k} \backslash\{\mathbf{0}\}$ was arbitrary.

\section{F $\quad$ Proof of Theorem 4.6}

When the subspace $\mathcal{L}$ described by $\boldsymbol{R}$ is fixed we can simplify our notation by regarding first $k$ components of $\boldsymbol{x}$ as $\boldsymbol{t}$ and the remaining components as $\boldsymbol{p}$. Then $\nabla \phi=\left[\boldsymbol{\psi}_{\boldsymbol{t}}, \boldsymbol{\psi}_{\boldsymbol{p}}\right]$. We then have the corresponding splitting of $\boldsymbol{P}=\left[\boldsymbol{P}_{1}, \boldsymbol{P}_{2}\right]$ and

$$
\boldsymbol{P}^{*}=W\left(\boldsymbol{F}+\left[\boldsymbol{\psi}_{\boldsymbol{t}}, \boldsymbol{\psi}_{\boldsymbol{p}}\right]\right)\left[\begin{array}{cc}
\boldsymbol{I}_{k} & \mathbf{0} \\
\mathbf{0} & \boldsymbol{I}_{d-k}
\end{array}\right]-\left[\begin{array}{c}
\boldsymbol{\psi}_{t}^{T} \\
\boldsymbol{\psi}_{\boldsymbol{p}}^{T}
\end{array}\right]\left[\boldsymbol{P}_{1}, \boldsymbol{P}_{2}\right]=\left[\begin{array}{cc}
\boldsymbol{P}_{1}^{*} & -\boldsymbol{\psi}_{\boldsymbol{t}}^{T} \boldsymbol{P}_{2} \\
-\boldsymbol{\psi}_{\boldsymbol{p}}^{T} \boldsymbol{P}_{1} & \boldsymbol{P}_{2}^{*}
\end{array}\right] .
$$

Similarly, splitting the $\boldsymbol{t}$ and $\boldsymbol{p}$ components we have

$$
\begin{gathered}
\widehat{\boldsymbol{P}}(\boldsymbol{H})=\left[\widehat{\boldsymbol{P}}_{1}, \widehat{\boldsymbol{P}}_{2}\right], \quad \widehat{\boldsymbol{P}}_{i}=\boldsymbol{P}_{i}\left(\boldsymbol{F}+\left[\boldsymbol{\psi}_{\boldsymbol{t}}, \boldsymbol{\psi}_{\boldsymbol{p}}\right]\right)-\boldsymbol{P}_{i}(\boldsymbol{F}), i=1,2 . \\
\widehat{\boldsymbol{P}}^{*}(\boldsymbol{H})=\left[\begin{array}{cc}
\widehat{\boldsymbol{P}}_{1}^{*} & -\boldsymbol{\psi}_{\boldsymbol{t}}^{T} \widehat{\boldsymbol{P}}_{2} \\
-\boldsymbol{\psi}_{\boldsymbol{p}}^{T} \widehat{\boldsymbol{P}}_{1} & \widehat{\boldsymbol{P}}_{2}^{*}
\end{array}\right]
\end{gathered}
$$

where

$$
\widehat{\boldsymbol{P}}_{1}^{*}=W^{\circ}\left(\boldsymbol{F},\left[\boldsymbol{\psi}_{\boldsymbol{t}}, \boldsymbol{\psi}_{\boldsymbol{p}}\right]\right) \boldsymbol{I}_{k}-\boldsymbol{\psi}_{\boldsymbol{t}}^{T} \widehat{\boldsymbol{P}}_{1} . \quad \widehat{\boldsymbol{P}}_{2}^{*}=W^{\circ}\left(\boldsymbol{F},\left[\boldsymbol{\psi}_{\boldsymbol{t}}, \boldsymbol{\psi}_{\boldsymbol{p}}\right]\right) \boldsymbol{I}_{d-k}-\boldsymbol{\psi}_{\boldsymbol{p}}^{T} \widehat{\boldsymbol{P}}_{2}
$$

Next we use the generalized Calpeyron's theorem [52] for $\widehat{W}(\boldsymbol{H})$ :

$$
\int_{|\boldsymbol{t}| \leq R} \int_{Q_{d-k}} \widehat{W}(\nabla \boldsymbol{\phi}) d \boldsymbol{p} d \boldsymbol{t}=\frac{1}{d}\left(T_{1}+T_{2}\right)
$$

where

$$
\begin{aligned}
& T_{1}=\int_{|\boldsymbol{t}|=R} \int_{Q_{d-k}}\left\{\left(\widehat{\boldsymbol{P}}_{1}^{*} \boldsymbol{n}_{\boldsymbol{t}}, \boldsymbol{t}\right)-\left(\boldsymbol{\psi}_{\boldsymbol{p}}^{T} \widehat{\boldsymbol{P}}_{1} \boldsymbol{n}_{\boldsymbol{t}}, \boldsymbol{p}\right)+\left(\widehat{\boldsymbol{P}}_{1} \boldsymbol{n}_{\boldsymbol{t}}, \boldsymbol{\psi}\right)\right\} d \boldsymbol{p} d S(\boldsymbol{t}), \\
& T_{2}=\int_{|\boldsymbol{t}| \leq R} \int_{\partial Q_{d-k}}\left\{\left(\widehat{\boldsymbol{P}}_{2}^{*} \boldsymbol{n}_{\boldsymbol{p}}, \boldsymbol{p}\right)-\left(\boldsymbol{\psi}_{\boldsymbol{t}}^{T} \widehat{\boldsymbol{P}}_{2} \boldsymbol{n}_{\boldsymbol{p}}, \boldsymbol{t}\right)+\left(\widehat{\boldsymbol{P}}_{2} \boldsymbol{n}_{\boldsymbol{p}}, \boldsymbol{\psi}\right)\right\} d S(\boldsymbol{p}) d \boldsymbol{t} .
\end{aligned}
$$

Next we observe that

$$
\int_{\partial Q_{d-k}} \boldsymbol{\psi}_{\boldsymbol{t}}^{T} \widehat{\boldsymbol{P}}_{2} \boldsymbol{n}_{\boldsymbol{p}} d S(\boldsymbol{p})=\mathbf{0}, \quad \int_{\partial Q_{d-k}}\left(\widehat{\boldsymbol{P}}_{2} \boldsymbol{n}_{\boldsymbol{p}}, \boldsymbol{\psi}\right) d S(\boldsymbol{p})=0,
$$

since $\boldsymbol{\psi}_{\boldsymbol{t}}^{T} \widehat{\boldsymbol{P}}_{2}$ and $\left(\widehat{\boldsymbol{P}}_{2}\right)^{T} \boldsymbol{\psi}$ are $Q_{d-k}$-periodic. By divergence theorem we obtain

$$
\int_{\partial Q_{d-k}}\left(\widehat{\boldsymbol{P}}_{2}^{*} \boldsymbol{n}_{\boldsymbol{p}}, \boldsymbol{p}\right) d S(\boldsymbol{p})=\int_{Q_{d-k}}\left\{\left(\nabla_{\boldsymbol{p}} \cdot \widehat{\boldsymbol{P}}_{2}^{*}, \boldsymbol{p}\right)+\operatorname{Tr} \widehat{\boldsymbol{P}}_{2}^{*}\right\} d \boldsymbol{p} .
$$


The Noether-Eshelby equation gives

$$
\nabla_{\boldsymbol{p}} \cdot \widehat{\boldsymbol{P}}_{2}^{*}=\nabla_{\boldsymbol{t}} \cdot\left(\boldsymbol{\psi}_{\boldsymbol{p}}^{T} \widehat{\boldsymbol{P}}_{1}\right)
$$

We also compute

$$
\operatorname{Tr} \widehat{\boldsymbol{P}}_{2}^{*}=(d-k) \widehat{W}-\operatorname{Tr}\left(\boldsymbol{\psi}_{\boldsymbol{p}}^{T} \widehat{\boldsymbol{P}}_{2}\right)
$$

Hence, we obtain

$$
T_{2}=(d-k) \int_{|\boldsymbol{t}| \leq R} \int_{Q_{d-k}} \widehat{W}(\nabla \boldsymbol{\phi}) d \boldsymbol{p} d \boldsymbol{t}+T_{2}^{\prime},
$$

where

$$
T_{2}^{\prime}=\int_{|\boldsymbol{t}|=R} \int_{Q_{d-k}}\left(\boldsymbol{\psi}_{\boldsymbol{p}}^{T} \widehat{\boldsymbol{P}}_{1} \boldsymbol{n}_{\boldsymbol{t}}, \boldsymbol{p}\right) d \boldsymbol{p} d S(\boldsymbol{t})-\int_{|\boldsymbol{t}| \leq R} \int_{Q_{d-k}} \operatorname{Tr}\left(\boldsymbol{\psi}_{\boldsymbol{p}}^{T} \widehat{\boldsymbol{P}}_{2}\right) d \boldsymbol{p} d \boldsymbol{t} .
$$

Substituting this back into (F.1) we obtain

$$
\int_{|\boldsymbol{t}| \leq R} \int_{Q_{d-k}} \widehat{W}(\nabla \boldsymbol{\phi}) d \boldsymbol{p} d \boldsymbol{t}=\frac{1}{k}\left(\widehat{T}_{1}(R)+\widehat{T}_{2}(R)\right),
$$

where

$$
\begin{gathered}
\widehat{T}_{1}(R)=\int_{|\boldsymbol{t}|=R} \int_{Q_{d-k}}\left\{\left(\widehat{\boldsymbol{P}}_{1}^{*} \boldsymbol{n}_{\boldsymbol{t}}, \boldsymbol{t}\right)+\left(\widehat{\boldsymbol{P}}_{1} \boldsymbol{n}_{\boldsymbol{t}}, \boldsymbol{\psi}\right)\right\} d \boldsymbol{p} d S(\boldsymbol{t}), \\
\widehat{T}_{2}(R)=-\int_{|\boldsymbol{t}| \leq R} \int_{Q_{d-k}} \operatorname{Tr}\left(\boldsymbol{\psi}_{\boldsymbol{p}}^{T} \widehat{\boldsymbol{P}}_{2}\right) d \boldsymbol{p} d \boldsymbol{t} .
\end{gathered}
$$

We observe that due to (4.17)

$$
\lim _{R \rightarrow \infty} \widehat{T}_{2}(R)=-\operatorname{Tr}\left(\int_{Y_{k}} \boldsymbol{\psi}_{\boldsymbol{p}}^{T} \widehat{\boldsymbol{P}}_{2} d \boldsymbol{p} d \boldsymbol{t}\right)=0 .
$$

To finish the proof of the theorem we need to show that $\widehat{T}_{1}(R) \rightarrow 0$, as $R \rightarrow \infty$.

We have $|\widehat{\boldsymbol{P}}| \leq C|\nabla \phi|$ and $\left|\widehat{\boldsymbol{P}}^{*}\right| \leq C|\nabla \phi|^{2}$, due to the uniform boundedness of $\nabla \boldsymbol{\phi}$, where the constant $C$ depends on $\phi$, but is independent of $R$. Thus, $\left|\widehat{T}_{1}(R)\right| \leq C K(R)$ for a.e. $R>1$, where

$$
K(R)=\int_{|\boldsymbol{t}|=R} \int_{Q_{d-k}}\left\{R|\nabla \boldsymbol{\phi}|^{2}+|\boldsymbol{\phi}-\boldsymbol{c}||\nabla \boldsymbol{\phi}|\right\} d \boldsymbol{p} d S(\boldsymbol{t}),
$$

where $\boldsymbol{c} \in \mathbb{R}^{m}$ can be chosen arbitrarily. We have, after an application of the Cauchy-Schwartz inequality

$$
\frac{1}{R} \int_{R}^{2 R} K(r) d r \leq 2 \int_{R<|\boldsymbol{t}|<2 R} \int_{Q_{d-k}}|\nabla \boldsymbol{\phi}|^{2} d \boldsymbol{p} d \boldsymbol{t}+\frac{\|\boldsymbol{\phi}-\boldsymbol{c}\|_{2,2 R}}{R}\left(\int_{R<|\boldsymbol{t}|<2 R} \int_{Q_{d-k}}|\nabla \boldsymbol{\phi}|^{2} d \boldsymbol{p} d \boldsymbol{t}\right)^{1 / 2} .
$$

If $k=1$ or $k=2$ then the boundedness of $\phi$ implies that

$$
\lim _{R \rightarrow \infty} \frac{1}{R} \int_{R}^{2 R} K(r) d r=0
$$

If $k \geq 3$ then Lemma C.1 guarantees the choice of the constant $\boldsymbol{c} \in \mathbb{R}^{m}$ such that (F.2) holds. Therefore,

$$
\varliminf_{R \rightarrow \infty} K(R)=0 .
$$

Hence,

$$
\left|\int_{Y} \widehat{W}(\nabla \phi) d \boldsymbol{x}\right| \leq C \varliminf_{R \rightarrow \infty} K(R)=0
$$

The theorem is proved. 


\section{G Proof of Lemma 5.5}

We will prove that $\mathcal{G}=\mathcal{G}_{0}$, where

$$
\mathcal{G}_{0}=\left\{\boldsymbol{A}=\operatorname{diag}\left(A_{1}, \ldots, A_{k}\right), A_{i}>0, i=1, \ldots, k, \operatorname{Tr} \boldsymbol{A}=1\right\} .
$$

If we write $\langle\Gamma(\boldsymbol{n})\rangle_{\boldsymbol{a}}$ in components

$$
\left(\langle\Gamma(\boldsymbol{n})\rangle_{\boldsymbol{a}}\right)_{i j}=f_{\mathbb{S}^{k-1}} \frac{n_{i} n_{j}}{a_{i} a_{j} \sum_{s=1}^{k} a_{s}^{-2} n_{s}^{2}} d S(\boldsymbol{n}) .
$$

we immediately see that $\mathcal{G} \subset \mathcal{G}_{0}$.

To each $\boldsymbol{a}=\left(a_{1}, \ldots, a_{k}\right) \in \mathbb{R}^{k}$ we associate (without relabeling) the diagonal matrix $\boldsymbol{a}=\operatorname{diag}\left(a_{1}, \ldots, a_{k}\right)$. Let $\Delta=\left\{\left(a_{1}, \ldots, a_{k}\right): a_{i}>0, \sum_{i=1}^{k} a_{i}=1\right\}$. Then the smooth map $F(\boldsymbol{a})=\langle\Gamma(\boldsymbol{n})\rangle_{\boldsymbol{a}}$ maps $\Delta$ into itself. To prove the reverse inclusion $\mathcal{G}_{0} \subset \mathcal{G}$ we need to show that the map $F: \Delta \rightarrow \Delta$ is surjective. We first show that the differential of the map $F$ is non-degenerate. This implies, via the inverse function theorem that $F(\Delta)$ is an open subset of $\Delta$.

In order to simplify the calculation we first change variables $\boldsymbol{b}=\boldsymbol{a}^{-1} / \operatorname{Tr}\left(\boldsymbol{a}^{-1}\right)$. Then

$$
F(\boldsymbol{a})=G\left(\frac{\boldsymbol{a}^{-1}}{\operatorname{Tr} \boldsymbol{a}^{-1}}\right), \quad G(\boldsymbol{b})=f_{\mathbb{S}^{k-1}} \frac{\boldsymbol{b} \boldsymbol{n} \otimes \boldsymbol{b} \boldsymbol{n}}{|\boldsymbol{b} \boldsymbol{n}|^{2}} d S(\boldsymbol{n}) .
$$

We compute

$$
d F(\boldsymbol{a}) \boldsymbol{\eta}=-d G\left(\frac{\boldsymbol{a}^{-1}}{\operatorname{Tr} \boldsymbol{a}^{-1}}\right) \frac{\boldsymbol{a}^{-1} \operatorname{Tr}\left(\boldsymbol{a}^{-1} \boldsymbol{\eta} \boldsymbol{a}^{-1}\right)-\boldsymbol{a}^{-1} \boldsymbol{\eta} \boldsymbol{a}^{-1} \operatorname{Tr} \boldsymbol{a}^{-1}}{\left(\operatorname{Tr} \boldsymbol{a}^{-1}\right)^{2}},
$$

where $\boldsymbol{\eta}$ is a diagonal trace-free matrix. If

$$
\boldsymbol{a}^{-1} \operatorname{Tr}\left(\boldsymbol{a}^{-1} \boldsymbol{\eta} \boldsymbol{a}^{-1}\right)-\boldsymbol{a}^{-1} \boldsymbol{\eta} \boldsymbol{a}^{-1} \operatorname{Tr} \boldsymbol{a}^{-1}=\mathbf{0}
$$

then $\boldsymbol{\eta}=\lambda \boldsymbol{a}$ for some scalar $\lambda$. Taking traces we conclude that $\lambda=0$. Hence, the map

$$
\boldsymbol{\eta} \mapsto \frac{\boldsymbol{a}^{-1} \operatorname{Tr}\left(\boldsymbol{a}^{-1} \boldsymbol{\eta} \boldsymbol{a}^{-1}\right)-\boldsymbol{a}^{-1} \boldsymbol{\eta} \boldsymbol{a}^{-1} \operatorname{Tr} \boldsymbol{a}^{-1}}{\left(\operatorname{Tr} \boldsymbol{a}^{-1}\right)^{2}}
$$

is a non-degenerate linear transformation on the space of diagonal trace-free matrices. Hence, $d F$ is nondegenerate if and only if $d G$ is non-degenerate. We compute explicitly

$$
d G \boldsymbol{\eta}=2 f_{\mathbb{S}^{k-1}}\left\{\frac{\boldsymbol{\eta n} \odot \boldsymbol{b} \boldsymbol{n}}{|\boldsymbol{b} \boldsymbol{n}|^{2}}-\frac{\boldsymbol{b} \boldsymbol{n} \otimes \boldsymbol{b n} \boldsymbol{n}}{|\boldsymbol{b n}|^{4}}(\boldsymbol{b n}, \boldsymbol{\eta n})\right\} d S(\boldsymbol{n}),
$$

where $\boldsymbol{\eta}$ is diagonal and $\operatorname{Tr} \boldsymbol{\eta}=0$. Suppose $d G \boldsymbol{\eta}=0$ for some non-zero $\boldsymbol{\eta}$ The Lemma will be proved if we show that only for $\boldsymbol{\eta}=\mathbf{0}$. If this is not the case then we have $\operatorname{Tr}\left(\boldsymbol{\eta} \boldsymbol{b}^{-1} d G \boldsymbol{\eta}\right)=0$. We compute (using commutativity of the diagonal matrix multiplication)

$$
\operatorname{Tr}\left(\boldsymbol{\eta} \boldsymbol{b}^{-1} d G \boldsymbol{\eta}\right)=2 \quad f_{\mathbb{S}^{k-1}} \frac{|\boldsymbol{\eta} \boldsymbol{n}|^{2}|\boldsymbol{b} \boldsymbol{n}|^{2}-(\boldsymbol{b} \boldsymbol{n}, \boldsymbol{\eta} \boldsymbol{n})^{2}}{|\boldsymbol{b n}|^{4}} d S(\boldsymbol{n}) .
$$

The Cauchy-Schwartz inequality implies that the integrand is non-negative. For it to be zero we would need $\boldsymbol{\eta n}=\alpha(\boldsymbol{n}) \boldsymbol{b} \boldsymbol{n}$ for almost all $\boldsymbol{n} \in \mathbb{S}^{k-1}$. The equivalent relation $\boldsymbol{b}^{-1} \boldsymbol{\eta} \boldsymbol{n}=\alpha(\boldsymbol{n}) \boldsymbol{n}$ means that every unit vector is an eigenvector of $\boldsymbol{b}^{-1} \boldsymbol{\eta}$. Hence, there is a constant $\alpha_{0}$ such that $\boldsymbol{\eta}=\alpha_{0} \boldsymbol{b}$. Taking the trace, we obtain $\alpha_{0}=0$ and the non-degeneracy of $d G$ is proved.

The lemma will follow, if we show that if $\boldsymbol{a}_{n} \rightarrow \boldsymbol{a}^{\circ} \in \partial \Delta$ and $F\left(\boldsymbol{a}_{n}\right) \rightarrow \boldsymbol{f}^{\circ}$, as $n \rightarrow \infty$ then $\boldsymbol{f}^{\circ} \in \partial \Delta$. Let $1 \leq i, j \leq k$ be a pair of indexes such that $a_{i}^{\circ}=0$ and $a_{j}^{\circ} \neq 0$. Such a pair exists, since $\boldsymbol{a}^{\circ} \in \partial \Delta$. We claim that $f_{j}^{\circ}=0$, finishing the proof of the lemma. We estimate

$$
F_{j}(\boldsymbol{a}) \leq f_{\mathbb{S}^{k-1}} \frac{a_{j}^{-2} n_{j}^{2}}{a_{j}^{-2} n_{j}^{2}+a_{i}^{-2} n_{i}^{2}} d S(\boldsymbol{n})=f_{\mathbb{S}^{k-1}} \frac{a_{i}^{2} n_{j}^{2}}{a_{i}^{2} n_{j}^{2}+a_{j}^{2} n_{i}^{2}} d S(\boldsymbol{n}) .
$$


Now, by the Lebesgue bounded convergence theorem,

$$
f_{j}^{\circ}=\lim _{n \rightarrow \infty} F_{j}\left(\boldsymbol{a}_{n}\right)=0 .
$$

Lemma 5.5 is now proved.

\section{References}

[1] R. Abeyaratne and J. Guo-Hua. Dilatationally nonlinear elastic materials-I. Some theory. International Journal of Solids and Structures, 25(10):1201 - 1219, 1989.

[2] S. Agmon, A. Douglis, and L. Nirenberg. Estimates near the boundary for solutions of elliptic partial differential equations satisfying general boundary conditions. I. Comm. Pure Appl. Math., 12:623-727, 1959.

[3] S. Agmon, A. Douglis, and L. Nirenberg. Estimates near the boundary for solutions of elliptic partial differential equations satisfying general boundary conditions. II. Comm. Pure Appl. Math., 17:35-92, 1964 .

[4] M. Alava, P. Nukala, and S. Zapperi. Statistical models of fracture. Advances in Physics, 55(3-4):349$476,2006$.

[5] G. Allaire. Shape optimization by the homogenization method, volume 146 of Applied Mathematical Sciences. Springer-Verlag, New York, 2002.

[6] G. Allaire and R. V. Kohn. Explicit optimal bounds on the elastic energy of a two-phase composite in two space dimensions. Quart. Appl. Math., LI(4):675-699, Dec. 1993.

[7] S. S. Antman. Nonlinear problems of elasticity, volume 107 of Applied Mathematical Sciences. Springer, New York, second edition, 2005.

[8] M. Baker and J. L. Ericksen. Inequalities restricting the form of the stress-deformation relations for isotropic elastic solids and Reiner-Rivlin fluids. J. Washington Acad. Sci., 44:33-35, 1954.

[9] J. Ball. Strict convexity, strong ellipticity, and regularity in the calculus of variations. In Math. Proc. Camb. Phil. Soc, volume 87, pages 501-513. Cambridge Univ Press, 1980.

[10] J. M. Ball. Convexity conditions and existence theorems in nonlinear elasticity. Arch. Ration. Mech. Anal., 63(4):337-403, 1976/77.

[11] J. M. Ball. Discontinuous equilibrium solutions and cavitation in nonlinear elasticity. Philos. Trans. Roy. Soc. London Ser. A, 306(1496):557-611, 1982.

[12] J. M. Ball. A version of the fundamental theorem for Young measures. In PDEs and continuum models of phase transitions (Nice, 1988), volume 344 of Lecture Notes in Phys., pages 207-215. Springer, Berlin-New York, 1989.

[13] J. M. Ball. Some open problems in elasticity. In Geometry, mechanics, and dynamics, pages 3-59. Springer, New York, 2002.

[14] J. M. Ball and J. E. Marsden. Quasiconvexity at the boundary, positivity of the second variation and elastic stability. Arch. Ration. Mech. Anal., 86(3):251-277, 1984.

[15] J. M. Ball and F. Murat. Remarks on Chacon's biting lemma. Proc. Amer. Math. Soc., 107(3):655-663, 1989. 
[16] P. W. Bates and P. C. Fife. The dynamics of nucleation for the Cahn-Hilliard equation. SIAM J. Appl. Math., 53(4):990-1008, 1993.

[17] V. L. Berdichevsky. Seed of a melt in a solid. Dokl. Acad. Nauk SSSR, 27:80-84, 1983.

[18] V. L. Berdichevsky. Variational principles of continuum mechanics. I Fundamentals. Springer-Verlag, Berlin, 2009.

[19] V. L. Berdichevsky. Variational principles of continuum mechanics. II Applications. Springer-Verlag, Berlin, 2009.

[20] M. A. Biot. Mechanics of incremental deformations. John Wiley \& Sons Inc., New York, 1965.

[21] B. Budiansky, J. Hutchinson, and J. Lambropoulos. Continuum theory of dilatant transformation toughening in ceramics. International Journal of Solids and Structures, 19(4):337 - 355, 1983.

[22] I. Chenchiah and K. Bhattacharya. The relaxation of two-well energies with possibly unequal moduli. Arch. Rat. Mech. Anal., 187(3):409-479, 2008.

[23] G. P. Cherepanov. Inverse problems of the plane theory of elasticity. J. Appl. Math. and Mech., 38(6):963-979, 1974.

[24] A. Cherkaev. Variational methods for structural optimization. Springer-Verlag, New York, 2000.

[25] A. Cherkaev and I. Kucuk. Detecting stress fields in an optimal structure. I. Two-dimensional case and analyzer. Struct. Multidiscip. Optim., 26(1-2):1-15, 2004.

[26] A. Cherkaev and I. Kucuk. Detecting stress fields in an optimal structure. II. Three-dimensional case. Struct. Multidiscip. Optim., 26(1-2):16-27, 2004.

[27] A. Cherkaev and Y. Zhang. Optimal anisotropic three-phase conducting composites: Plane problem. International Journal of Solids and Structures, 48(20):2800-2813, Oct. 2011.

[28] M. Chipot and D. Kinderlehrer. Equilibrium configurations of crystals. Arch. Rational Mech. Anal., 103(3):237-277, 1988.

[29] P. G. Ciarlet. Mathematical elasticity. Vol. I: Three-dimensional elasticity, volume 20 of Studies in Mathematics and its Applications. North-Holland Publishing Co., Amsterdam, 1988.

[30] M. G. Crandall and P. H. Rabinowitz. Bifurcation from simple eigenvalues. J. Functional Analysis, 8:321-340, 1971.

[31] B. Dacorogna. Necessary and sufficient conditions for strong ellipticity of isotropic functions in any dimension. Discrete And Continuous Dynamical Systems, Series B, 1(2):257-263, 2001.

[32] G. Dolzmann. Variational methods for crystalline microstructure: analysis and computation, volume 1803 of Lecture Notes in Mathematics. Springer Verlag, 2003.

[33] W. S. Edelstein and R. L. Fosdick. A note on non-uniqueness in linear elasticity theory. Zeitschrift für Angewandte Mathematik und Physik (ZAMP), 19:906-912, 1968. 10.1007/BF01602270.

[34] G. Erdmann. Über die unstetige Lösungen in der Variationsrechnung. J. Reine Angew. Math., 82:21-30, 1877 .

[35] J. L. Ericksen and R. A. Toupin. Implications of Hadamard's conditions for elastic stability with respect to uniqueness theorems. Canad. J. Math., 8:432-436, 1956. 
[36] J. D. Eshelby. The determination of the elastic field of an ellipsoidal inclusion, and related problems. Proceedings of the Royal Society of London. Series A, Mathematical and Physical Sciences, 241:376-396, 1957.

[37] J. D. Eshelby. The elastic field outside an ellipsoidal inclusion. Proceedings of the Royal Society of London. Series A, Mathematical and Physical Sciences, 252:561-569, 1959.

[38] J. D. Eshelby. Energy relations and energy momentum tensor in continuum mechanics. In M. Kanninen, W. Adler, A. Rosenfeld, and R. Jaffee, editors, Inelastic behavior of solids, pages 77-114. McGraw-Hill, New York, 1970.

[39] J. D. Eshelby. The elastic energy-momentum tensor. Journal of Elasticity, 5(3-4):321-335, 1975.

[40] A. B. Freidin. On new phase inclusions in elastic solids. Z. Angew. Math. Mech., 87(2):102-116, 2007.

[41] A. B. Freidin and A. M. Chiskis. Zones of phase transitions in non-linear elastic isotropic materials. Part 1. Basic relations. Izv Akad Nauk MTT (Mechanics of Solids), 4:91-109, 1994. In Russian.

[42] A. B. Freidin and A. M. Chiskis. Zones of phase transitions in non-linear elastic isotropic materials. Part 2. Incompressible materials with a potentials solely dependent on one of the invariants of the strain tensor. Izv Akad Nauk MTT (Mechanics of Solids), 5:49-61, 1994. In Russian.

[43] I. M. Gelfand and S. V. Fomin. Calculus of variations. Prentice-Hall, 1963.

[44] G. Geymonat, S. Müller, and N. Triantafyllidis. Homogenization of non-linearly elastic materials, microscopic bifurcation and macroscopic loss of rank-one convexity. Arch. Ration. Mech. Anal., 122(3):231290, 1993.

[45] M. Giaquinta and S. Hildebrandt. Calculus of variations. I The Lagrangian formalism, volume 310 of Grundlehren der Mathematischen Wissenschaften. Springer-Verlag, Berlin, 1996.

[46] Y. Grabovsky. Nonsmooth analysis and quasi-convexification in elastic energy minimization problems. Structural Optimization, 10(3/4):217-221, Dec. 1995.

[47] Y. Grabovsky. Bounds and extremal microstructures for two-component composites: A unified treatment based on the translation method. Proc. Roy. Soc. London, Series A., 452(1947):945-952, 1996.

[48] Y. Grabovsky and R. V. Kohn. Anisotropy of the vigdergauz microstructure. J. Appl. Mech., 62(4):1063$1065,1995$.

[49] Y. Grabovsky, V. Kucher, and L. Truskinovsky. Probing the limits of rank-one convexity. in preparation.

[50] Y. Grabovsky and L. Truskinovsky. The flip side of buckling. Cont. Mech. Thermodyn., 19(3-4):211-243, 2007.

[51] Y. Grabovsky and L. Truskinovsky. Roughening instability of broken extremals. Arch. Rat. Mech. Anal., 200(1):183-202, 2011.

[52] Y. Grabovsky and L. Truskinovsky. Generalized clapeyron's theorem. in preparation.

[53] Y. Grabovsky and L. Truskinovsky. Metastability in an elastic material with incompatible energy wells: an example. in preparation.

[54] Y. Grabovsky and L. Truskinovsky. Metastability in the presence of phase boundaries. in preparation.

[55] M. E. Gurtin. Configurational forces as basic concepts of continuum physics, volume 137 of Applied Mathematical Sciences. Springer-Verlag, New York, 2000. 
[56] S. Gutiérrez. Laminations in linearized elasticity: the isotropic non-very strongly elliptic case. $J$. Elasticity, 53(3):215-256, 1998/99.

[57] R. Hill. On uniqueness and stability in the theory of finite elastic strain. J. Mech. Phys. Solids, $5: 229-241,1957$.

[58] R. Hill. On the elasticity and stability of perfect crystals at finite strain. Math. Proc. Cambridge Philos. Soc., 77:225-240, 1975.

[59] E. Hohlfeld and L. Mahadevan. Unfolding the sulcus. Phys. Rev. Lett., 106:105702, Mar 2011.

[60] I. M. Kaganova and A. L. Roytburd. Equilibrium between elastically-interacting phases. Sov. Phys. JETP, 67(6):1173-1183, 1988.

[61] A. G. Khachaturyan. Theory of structural transformation in solids. Wiley, New York, 1983.

[62] D. Kinderlehrer. Remarks about equilibrium configurations of crystals. In Material instabilities in continuum mechanics (Edinburgh, 1985-1986), Oxford Sci. Publ., pages 217-241. Oxford Univ. Press, New York, 1988.

[63] J. K. Knowles and E. Sternberg. On the ellipticity of the equations of nonlinear elastostatics for a special material. Journal of Elasticity, 5:341-361, 1975.

[64] J. K. Knowles and E. Sternberg. On the failure of ellipticity and the emergence of discontinuous deformation gradients in plane finite elastostatics. J. Elasticity, 8(4):329-379, 1978.

[65] H. Knüpfer, R. Kohn, and F. Otto. Nucleation barriers for the cubic-to-tetragonal transformation. Comm Pure Appl Math, 2012.

[66] H. Knüpfer and R. V. Kohn. Minimal energy for elastic inclusions. Proceedings of the Royal Society A: Mathematical, Physical and Engineering Science, 467(2127):695-717, 2011.

[67] R. V. Kohn. The relaxation of a double-well energy. Continuum Mech. Thermodyn., 3:193-236, 1991.

[68] R. V. Kohn and G. Strang. Optimal design and relaxation of variational problems. Comm. Pure Appl. Math., 39:113-137, 139-182 and 353-377, 1986.

[69] J. Kristensen. On the non-locality of quasiconvexity. Annales de l'Institut Henri Poincare (C) Non Linear Analysis, 16(1):1 - 13, 1999.

[70] L. B. Kublanov and A. B. Freidin. Nuclei of a solid phase in a deformable material. Prikl. Mat. Mekh., 52(3):493-501, 1988.

[71] I. Kunin, G. Mirenkova, and E. Sosnina. An ellipsoidal crack and needle in an anisotropic elastic medium: Pmm vol. 37, n 3, 1973, pp. 524-531. Journal of Applied Mathematics and Mechanics, 37(3):501-508, 1973.

[72] I. Kunin and É. Sosnina. Ellipsoidal inhomogeneity in an elastic medium. In Soviet Physics Doklady, volume 16, page 534, 1971.

[73] I. Kunin and E. Sosnina. Stress concentration on an ellipsoidal inhomogeneity in an anisotropic elastic medium. Prikladnaia Matematika i Mekhanika, 37:306-315, 1973.

[74] J. Langer. Metastable states. Physica, 73(1):61 - 72, 1974.

[75] H. Le Dret. An example of $H^{1}$-unboundedness of solutions to strongly elliptic systems of partial differential equations in a laminated geometry. Proc. Roy. Soc. Edinburgh Sect. A, 105:77-82, 1987. 
[76] J. Li, T. Zhu, S. Yip, K. J. V. Vliet, and S. Suresh. Elastic criterion for dislocation nucleation. Materials Science and Engineering: A, 365(1-2):25 - 30, 2004.

[77] I. M. Lifshits and L. S. Gulida. On nucleation under local melting. Dokl. Akad. Nauk SSSR, 87(4):523526, 1952. in Russian, English version available.

[78] I. M. Lifshits and L. S. Gulida. On the theory of local melting. Dokl. Akad. Nauk SSSR, 87(3):377-380, 1952. in Russian, English version available.

[79] J. Lu. Elastic energy minimization and the shape of coherent precipitates. PhD thesis, New York University, New York, NY, 1993.

[80] K. A. Lurie. Optimum control of conductivity of a fluid moving in a channel in a magnetic field. $J$. Appl. Math. Mech., 28(2):316-327, 1964.

[81] K. A. Lurie. Applied optimal control theory of distributed systems. Plenum Press, New York, 1993.

[82] C. Maloney and A. Lemaitre. Universal breakdown of elasticity at the onset of material failure. Physical review letters, 93(19):195501, 2004.

[83] G. A. Maugin. Material inhomogeneities in elasticity. Chapman and Hall, Ltd., London, 1993.

[84] J. C. Michel, O. Lopez-Pamies, P. Ponte Castañeda, and N. Triantafyllidis. Microscopic and macroscopic instabilities in finitely strained porous elastomers. J. Mech. Phys. Solids, 55(5):900-938, 2007.

[85] A. Mielke and P. Sprenger. Quasiconvexity at the boundary and a simple variational formulation of Agmon's condition. J. Elasticity, 51(1):23-41, 1998.

[86] C. B. Morrey, Jr. Quasi-convexity and the lower semicontinuity of multiple integrals. Pacific J. Math., 2:25-53, 1952.

[87] M. Morse. The calculus of variations in the large, volume 18. Amer Mathematical Society, 1934.

[88] S. Müller, J. Sivaloganathan, and S. J. Spector. An isoperimetric estimate and $W^{1, p}$-quasiconvexity in nonlinear elasticity. Calc. Var. Partial Differential Equations, 8(2):159-176, 1999.

[89] S. Müller and V. Šverák. Convex integration for Lipschitz mappings and counterexamples to regularity. Ann. of Math. (2), 157(3):715-742, 2003.

[90] T. Mura. Micromechanics of defects in solids. Springer, 1987.

[91] N. I. Muskhelishvili. Some basic problems of the mathematical theory of elasticity. P. Noordhoff Ltd., Groningen-Holland, 1953.

[92] E. Noether. Invariante variationsprobleme. Nachr. v. d. Ges. d. Wiss. zu Göttingen, pages 235-257, 1918. English translation in "Transport Theory and Statistical Mechanics" 1(3):183-207, 1971.

[93] R. Ogden. Non-linear elastic deformations. Dover Pubns, 1997.

[94] P. Pedregal. Parametrized measures and variational principles. Progress in Nonlinear Differential Equations and their Applications, 30. Birkhäuser Verlag, Basel, 1997.

[95] P. Pedregal. Fully explicit quasiconvexification of the mean-square deviation of the gradient of the state in optimal design. Electron. Res. Announc. Amer. Math. Soc., 7:72-78 (electronic), 2001.

[96] K. A. Pericak-Spector, J. Sivaloganathan, and S. J. Spector. An explicit radial cavitation solution in nonlinear elasticity. Math. Mech. Solids, 7(1):87-93, 2002. 
[97] A. Roytburd and J. Slutsker. Deformation of adaptive materials. Part I: Constrained deformation of polydomain crystals. Journal of the Mechanics and Physics of Solids, 47:2299-2329, 1999.

[98] A. Roytburd and J. Slutsker. Deformation of adaptive materials. Part II: Adaptive composite. Journal of the Mechanics and Physics of Solids, 47:2331-2349, 1999.

[99] A. Roytburd and J. Slutsker. Deformation of adaptive materials. Part III: Deformation of crystals with polytwin product phases. Journal of the Mechanics and Physics of Solids, 49(8):1795-1822, Aug 2001.

[100] O. Salman and L. Truskinovsky. On the critical nature of plastic flow: One and two dimensional models. International Journal of Engineering Science, 2012.

[101] J. Sethna. Crackling noise and avalanches: Scaling, critical phenomena, and the renormalization group. Les Houches, 85:257-288, 2007.

[102] M. Šilhavý. The mechanics and thermodynamics of continuous media. Springer-Verlag, Berlin, 1997.

[103] H. C. Simpson and S. J. Spector. On barrelling instabilities in finite elasticity. J. Elasticity, 14(2):103$125,1984$.

[104] H. C. Simpson and S. J. Spector. On the positivity of the second variation in finite elasticity. Arch. Ration. Mech. Anal., 98(1):1-30, 1987.

[105] H. C. Simpson and S. J. Spector. Necessary conditions at the boundary for minimizers in finite elasticity. Arch. Ration. Mech. Anal., 107(2):105-125, 1989.

[106] H. C. Simpson and S. J. Spector. On bifurcation in finite elasticity: buckling of a rectangular rod. $J$. Elasticity, 92(3):277-326, 2008.

[107] J. Sokolowski and J.-P. Zolesio. Introduction to Shape Optimization. Shape Sensitivity Analysis. Springer-Verlag, 1992.

[108] E. M. Stein. Singular integrals and differentiability properties of functions. Princeton Mathematical Series, No. 30. Princeton University Press, Princeton, N.J., 1970.

[109] C. A. Stuart. Radially symmetric cavitation for hyperelastic materials. Ann. Inst. H. Poincaré Anal. Non Linéaire, 2(1):33-66, 1985.

[110] T. Tanaka, S.-T. Sun, Y. Hirokawa, S. Katayama, J. Kucera, Y. Hirose, and T. Amiya. Mechanical instability of gels at the phase transition. Nature, 325(6107):796-798, 021987.

[111] L. Tartar. The general theory of homogenization, volume 7 of Lecture Notes of the Unione Matematica Italiana. Springer-Verlag, Berlin, 2009. A personalized introduction.

[112] C. Truesdell and W. Noll. The non-linear field theories of mechanics. Springer-Verlag, Berlin, third edition, 2004.

[113] L. Truskinovsky and G. Zanzotto. Ericksen's bar revisited: energy wiggles. J. Mech. Phys. Solids, 44(8):1371-1408, 1996.

[114] L. M. Truskinovsky. Dynamics of nonequilibrium phase boundaries in a heat conducting non-linearly lastic medium. Prikl. Mat. Mekh., 51(6):1009-1019, 1987.

[115] J. van der Waals. The equilibrium between a solid body and a fluid phase, especially in the neighbourhood of the critical state. In KNAW, Proceedings, volume 6, pages 1903-1904, 1903. 
[116] L. Van Hove. Sur l'extension de la condition de Legendre du calcul des variations aux intégrales multiples à plusieurs fonctions inconnues. Nederl. Akad. Wetensch., Proc., 50:18-23, 1947. (Indagationes Math. $9,3-8,1947)$.

[117] S. B. Vigdergauz. Integral equation of the inverse problem of the plane theory of elasticity. Prikladnaia Matematika i Mekhanika (PMM), 40(3):518-521, 1976.

[118] S. B. Vigdergauz. On a case of the inverse problem of two-dimensional theory of elasticity. Prikladnaia Matematika i Mekhanika (PMM), 41(5):927-933, 1977.

[119] S. B. Vigdergauz. Regular structures with extremal elastic properties. MTT, 24(3):57-63, 1989.

[120] S. B. Vigdergauz. Two-dimensional grained composites of extreme rigidity. ASME J. Appl. Mech., 61(2):390-394, 1994.

[121] L. C. Young. Generalized curves and the existence of an attained absolute minimum in the calculus of variations. Comptes Rendue Soc. Sciences \&3 Lettres Varsovie, cl. III, 30:212-234, 1937.

[122] L. C. Young. Generalized surfaces in the calculus of variations. Ann. of Math. (2), 43(1):84-103, 1942.

[123] L. C. Young. Lectures on the calculus of variations and optimal control theory. W. B. Saunders Co., Philadelphia, 1969. Foreword by Wendell H. Fleming.

[124] M. Zaiser. Scale invariance in plastic flow of crystalline solids. Advances in Physics, 55(1-2):185-245, 2006.

[125] L. Zee and E. Sternberg. Ordinary and strong ellipticity in the equilibrium theory of incompressible hyperelastic solids. Archive for Rational Mechanics and Analysis, 83:53-90, 1983. 10.1007/BF00281087.

[126] Z. Zhang, R. D. James, and S. Müller. Energy barriers and hysteresis in martensitic phase transformations. Acta Materialia, 57(15):4332 - 4352, 2009. 